\author{
Universidade de São Paulo \\ Instituto de Física
}

\title{
Fenomenologia de Teorias Quiver da Quebra da Simetria Eletrofraca
}

\author{
Gabriela Lima Lichtenstein
}

Orientador: Prof. Dr. Gustavo A. Burdman

Dissertação de mestrado apresentada ao Instituto de Física para a obtenção do título de Mestre em Ciências

Banca Examinadora:

Prof. Dr. Gustavo A. Burdman Orientador (Instituto de Física, USP)

Prof. Dr. Oscar J. P. Eboli (Instituto de Física, USP)

Prof. Dr. Pedro Galli Mercadante (CCNH, UFABC)

São Paulo

2014 



\section{Sumário}

1 Introdução: Física Além do Modelo Padrão 1

1.1 Modelo Padrão da Física de Partículas . . . . . . . . . . . . . . . . . . . . 2

1.1.1 Quebra espontânea de Simetria . . . . . . . . . . . . . . . . 3

1.1 .2 Mecanismo de Higgs . . . . . . . . . . . . . . . . . . 6

1.1.3 Teoria de interações eletrofracas . . . . . . . . . . . . . 8

1.1 .4 Férmions no MP . . . . . . . . . . . . . . . . . . 10

1.1.5 Termo de Yukawa das massas dos férmions . . . . . . . . . . 10

1.1.6 Testes experimentais . . . . . . . . . . . . . . . . 11

1.2 Física Além do Modelo Padrão . . . . . . . . . . . . . . . . . . . . . 13

1.2.1 Problema de hierarquia de gauge . . . . . . . . . . . . . . 14

1.2.2 Teorias Quiver como solução ao problema de hierarquia . . . . . 16

2 Teorias Quiver 17

2.1 Quebra de simetria em Teorias Quiver . . . . . . . . . . . . . . . . . 18

2.1.1 Função de onda dos bósons no modo zero . . . . . . . . . . . . 23

2.2 Inclusão dos férmions na Teoria Quiver . . . . . . . . . . . . . . . . . 24

2.3 Limite do Contínuo . . . . . . . . . . . . . . . . . . . . . 27

2.3.1 Randall-Sundrum . . . . . . . . . . . . . . . . . 27

2.3.2 Desconstrução dimensional . . . . . . . . . . . . . . . 28

2.3.3 Localização das funções de onda dos férmions . . . . . . . . . . . 32

3 Acoplamento dos estados excitados $\quad 35$

3.1 Acoplamentos para o modo zero dos férmions . . . . . . . . . . . . . 35

3.1.1 Acoplamentos em função da localização . . . . . . . . . . . . . 36

3.1 .2 Acoplamento de Yukawa . . . . . . . . . . . . . . . . 38

3.2 Localização do Higgs . . . . . . . . . . . . . . . . . . . . . . . . . 39

3.3 Localização dos férmions no Quiver . . . . . . . . . . . . . . . . . . . 43

3.3.1 Correntes neutras com mudança de sabor . . . . . . . . . . . 43

3.3.2 Violação de sabor no Quiver . . . . . . . . . . . . . . . . 45

4 Fenomenologia $\quad 51$

4.1 Seção de Choque partônica . . . . . . . . . . . . . . 52

4.1 .1 Largura . . . . . . . . . . . . . . . . . . 52 
4.1 .2 Seção de Choque Partônica . . . . . . . . . . . . . . . . . . 55

4.2 Seção de Choque $p p \rightarrow G^{(1)} \rightarrow q \bar{q} \ldots \ldots \ldots \ldots \ldots$

4.2.1 Funções de Distribuição Partônica . . . . . . . . . . . . . . . . . . 59

4.3 Procura no LHC . . . . . . . . . . . . . . . . . . . . . . 61

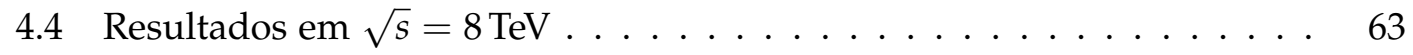

4.5 Fenomenologia no LHC . . . . . . . . . . . . . . . . . . 65

4.5 .1 Vínculos experimentais . . . . . . . . . . . . . . . 65

4.5 .2 Perspectivas no LHC . . . . . . . . . . . . . . . . . . . 68

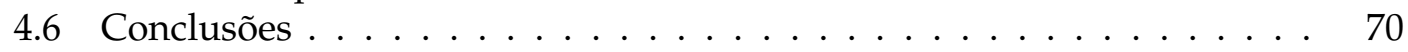

5 Conclusões Finais $\quad \mathbf{7 1}$

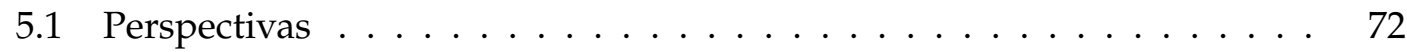




\section{Resumo}

O Modelo Padrão (MP) da Física de Partículas é uma descrição extremamente bem sucedida dos dados experimentais com que contamos hoje. No entanto, as suas limitações são aparentes pelo fato dele possuir um grande número de parâmetros arbitrários. Mais importante ainda, no MP a escala eletrofraca é instável, o que dá lugar ao chamado problema da hierarquia de escalas. A solução desse problema requer nova física na escala $\mathrm{TeV}$, que é precisamente a escala de energia a ser explorada no Large Hadron Collider (LHC) do CERN. Neste trabalho foi estudada a fenomenologia de Teorias Quiver, que são extensões do Modelo Padrão (MP) que descrevem nova física na escala TeV.

Foram investigadas teorias da escala eletrofraca onde a hierarquia com a escala ultravioleta, ou seja, a escala de Planck, é gerada por uma estrutura de cadeia de grupos de gauge espontaneamente quebrados em cascata. Essas teorias, também geram a hierarquia das massas dos férmions de forma natural, mas são puramente quadridimensionais. Porém, elas podem ser construídas a partir de teorias com uma dimensão extra curva, com métrica anti de Sitter. Desta forma, as teorias resultantes tem várias propriedades boas das teorias com dimensões extras, mas são mais gerais e melhor comportadas no ultra-violeta.

Foram calculados os acoplamentos do primeiro estado excitado dos bósons de gauge com quarks do MP. É possível determinar a localização dos férmions no Quiver, analogamente à localização na dimensão extra. Deste modo, define-se os acoplamentos para um certo número de sítios.

Esse trabalho tem como objetivo o estudo da fenomenologia das chamadas Teorias Quiver no LHC. Foram obtidos vínculos de massa para ressonâncias na escala TeV. Tanto dados vindo de experimentos de precisão eletrofracos quanto de detecção direta restringem a teoria para a massa do estado excitado do bóson de gauge a $M_{G}>$ $3 \mathrm{TeV}$. Dados vindos da próxima fase do LHC, com maior energia e luminosidade, irão resultar em vínculos de detecção direta que ultrapassam os limites dos vínculos indiretos de sabor e precisão eletrofraca. 



\begin{abstract}
The Standard Model of Particle Physics (SM) is a very successful description of the experimental data gathered until now. However, this model has limitations due to the large number of arbitrary parameters. More importantly, the electroweak scale is unstable, which results in the scale hierarchy problem. The solution to this problem requires new physics at the $\mathrm{TeV}$ scale, which is precisely the energy scale to be explored at the Large Hadron Collider (LHC) at CERN. We have studied the phenomenolgy of Full Hierarchy Quiver Theories (FHQT), which is an extension of the Standard Model (SM) that describes new physics at the TeV scale.

We have investigated theories of the electroweak scale, where the hierarchy with the ultraviolet scale (e.g. Plank scale) is generated by a chain of gauge groups spontaneously broken by ordered vacuum expectation values. These four-dimensional quantum field theories also generate the fermion mass hierarchy in a natural way. They can be obtained by deconstruction of models with a curved extra dimension in an anti-de Sitter backround. Thus, the resultant four-dimensional theories have many of the desired properties that extra dimensional theories have, alas without some of its problems.

We have calculated the couplings acquired from the interaction between the first excited mode of the gauge boson with SM quarks. It is possible to obtain the localization of the wave function of the fermion zero modes in a similar way it is done in extra dimensional theories. Thus the couplings depend on the number of sites in the theory.

This project has the purpose of studying the phenomenology of what we call full hierarchy quiver theories (FHQT). We obtained bounds of resonance masses at the $\mathrm{TeV}$ scale. Both data from electroweak precision measurements and direct detection result in $M_{G}>3 \mathrm{TeV}$. Data from the next run at the LHC, at higher energies and luminosities, will result in the first direct search bounds that surpass indirect flavor and electroweak precision limits.
\end{abstract}





\section{Agradecimentos}

Dedico essa dissertação a todos que conviveram comigo durante sua construção e me incentivaram a prosseguir nos estudos. Primeiramente, agradeço ao meu orientador Gustavo Burdman quem admiro muito, sou grata a sua compreenção, às conversas e todo o conhecimento que sempre compartilhou com entusiasmo. Também à Nayara Fonseca de Sá e Leonardo de Lima por terem tirado minhas dúvidas e por toda a ajuda com a física.

Â minha família pelo amor e apoio incondicional, especialmente aos meus pais, Paul e Tamara. Dedidico também à Vovó Esthela por estar sempre presente e ser uma grande companheira. À Thais por ser sempre querida. Agradeço os incentivos de todos os tios e primos, principalmente a Beth, Tonico, Cassio, Thiago e Patricia. Além de ser sempre grata à Gracinha e Célia, que aturaram toda a minha bagunça.

Há duas pessoas que foram fundamentais, pois me ajudaram a me organizar e colaboraram para que eu crescesse. Julieta, que me ajudou a encontrar minhas prórias palavras. E Vera, sem a qual eu não estaria em pé. Também agradeço à Denise Demange que, além de grande amiga, me ajudou a organizar minhas idéias. Ao Mário Marino, que me ensinou a superar minhas dificuldades.

Aos amigos que foram meus companheiros. Tanto as que sempre estiveram presente Carla, Carol, Dani e Cláu (tchurma). Quanto amigos mais recentes Thiago, Leila, Maria Fernanda, Alysson, Carol, Javier, Pablo, Meera, Daniel, Michele, Wilson, Leandro. Assim como aos colegas e professores de graduação do ciências moleculares que eu sempre me lembro com carinho.

Finalmente, agradeço à CAPES apoio financeiro. 



\section{Epígrafe}

"Os Distantes defendiam uma ideia oposta: por trás de todos os físicos e astrônomos jazia o corpo inerte, mas não morto - apenas em animação suspensa - de um contador de histórias que sucumbiu à tentação cósmica de cifras e fórmulas."Página 17, O Fundo do Céu, Rodrigo Fresán. COSACNAIFY 



\section{1}

\section{Introdução: Física Além do Modelo Padrão}

A Física de Partículas é descrita pelo Modelo Padrão (MP). Ele consiste em uma teoria de gauge, na qual as massas dos bósons eletrofracos são obtidas a partir do mecanismo de Higgs. A teoria é muito bem estabelecida experimentalmente, descrevendo com precisão as partículas elementares até hoje observadas. Tendo como mais recente descoberta confirmação da existência de uma nova ressonância a $125 \mathrm{GeV}$, o bóson de Higgs no Large Hadron Collider (LHC), no CERN (Centre European des Recherches Nucléaires).

No entanto, restam algumas perguntas a serem respondidas pelo MP da Física de Partículas, sendo o enfoque deste estudo a fenomenologia associada a uma possível solução ao problema de hierarquia. O MP não explica todos os aspectos da física de partículas, o que será discutido no final desse capítulo. Uma das questões além do MP é o problema de hierarquia, o qual é importante pois implica na existência nova física na escala $\mathrm{TeV}$. Ele consiste na questão de que há uma enorme discrepância entre as escalas eletrofraca e ultravioleta, onde o MP é válido. Essa diferença pode chegar a $10^{17}$ ordens de grandeza ao considerar a escala de Planck, onde interações gravitacionais devem ser incluídas. O problema de hierarquia implica que deve haver um ajuste fino na teoria ou física nova além do MP. Existem diversos modelos na literatura candidatos a solucionar esse problema, entre eles Supersimetria, Little Higgs ou teorias com dimensões extras.

Nesse trabalho serão estudadas Teorias Quiver como candidatas a resolver o problema de hierarquia. Elas são modelos puramente quadridimensionais, contendo uma cadeia de grupos de gauge conectados por campos de ligação. Esses campos quebram dinamicamente a simetria gerando naturalmente a hierarquia de massas dos férmions, o que será explicado em mais detalhe no capítulo 2. Além disso, será descrito o Higgs como pseudo bóson de Nambu Goldstone no capítulo 3 a fim de resolver o problema de hierarquia.

O objetivo dessa dissertação é estudar a fenomenologia de Teorias Quiver. Essa análise está publicada na referência [1]. Foram obtidos vínculos às massas dos modos exci- 
tados dos bósons de gauge a partir de dados acumulados do LHC.

A dissertação está dividida de modo que o primeiro capítulo introduz conceitos bem estabelecidos do MP da física de partículas. Serão descritos aspectos teóricos necessários para sua compreensão, assim como as questões além do MP, principalmente o problema de hierarquia de gauge e das massas dos férmions.

O capítulo 2 descreve as Teorias Quiver. Será apresentado um modelo contendo $\mathrm{N}$ grupos de gauge conectados por campos de ligação. A quebra da simetria desses gera uma torre de modos excitados dos bósons de gauge. Além disso, a quebra dinâmica dará lugar à hierarquia das massas dos férmions. Essas teorias são candidatas a resolver o problema de hierarquia, sendo alternativas às teorias com dimensões extras. No entanto, é necessário ressaltar que são modelos puramente quadridimensionais, apesar de poderem ser desconstruídas de um cenário $A d S_{5}$. Os modelos diferem e pode-se gerar uma fenomenologia distinta em Teorias Quiver com poucos sítios.

O capítulo 3 contém os cálculos necessários para o estudo da fenomenologia. Serão calculados os acoplamentos dos quarks do modelo padrão com o primeiro modo excitado dos bósons de gauge. Esses acoplamentos caracterizam a Teoria Quiver no cálculo da seção de choque. Além disso, será descrito o Higgs como um pseudo Bóson de Nambu Goldstone a fim de resolver o problema de hierarquia. Também é mostrado que esse modelo tem mínima violação de sabor a nível árvore.

No capítulo 4 são obtidos os vínculos experimentais com dados acumulados pelo LHC, apresentados em [1] . Será calculada a seção de choque de produção e decaimento em quarks do modo um dos bósons de gauge, a qual será comparada com dados obtidos pelas colaborações CMS e ATLAS em procura por nova física, apresentados nas referências [2], [3] e [4].

Finalmente, as conclusões e as possibilidades futuras são apresentadas no capítulo 5

\subsection{Modelo Padrão da Física de Partículas}

O Modelo Padrão da Física de Partículas descreve as interações das partículas elementares observadas até agora. Através do mecanismo de Higgs são geradas as massas dos bósons eletrofracos. Nele os bósons de Nambu Goldstone (bNG) são absorvidos pelos bósons de gauge, sobrando um grau de liberdade correspondente ao bóson de Higgs. O MP eletrofraco é descrito a partir da quebra espontânea da simetria $S U(2)_{L} \times U(1)_{Y} \rightarrow U(1)_{E M}$ o que gera massa aos bósons $W^{ \pm}$e $Z^{0}$, restando um estado ortogonal não massivo correspondente ao fóton $\gamma$. As simetrias de cor $S U(3)_{C}$ são expectadores da quebra espontânea, as interações fortes são descritas pela QCD através de interações entre quarks e glúons. Além disso, as 3 famílias de férmions são descritas por dubletos de leptons e quarks sob $S U(2)_{L}$ para férmions de mão esquerda, e os correspondentes singletos de $S U(2)_{R}$ para os de mão direita. 


\subsubsection{Quebra espontânea de Simetria}

A fim de descrever o MP da Física de partículas será necessário descrever a quebra espontânea de simetria, o que pode ser encontrado em referêcias como [5] ou [6]. Esse mecanismo é utilizado para descrever as massas dos bósons de gauge. O MP será demonstrado a partir do mecanismo de Higgs, o qual é equivalente à quebra espontânea de uma simetria de gauge.

Os modelos em Física de Partículas são descritos a partir do grupo de simetria, sendo que a invariância de gauge local descreve a dinâmica de interações na Teoria Quântica de Campos. Isso é possível graças ao Teorema de Noether, o qual afirma que simetrias contínuas implicam em leis de conservação na física. Em termos da Teoria de Campos ele também pode ser aplicado. Neste caso, para qualquer invariância da ação sob uma transformação contínua dos campos, existe uma carga associada a uma corrente conservada, $\partial_{\mu} J^{\mu}=0$.

Entretanto, termos de massa não são invariantes sob a simetria de gauge. Logo, é possível descrever uma teoria apenas com bósons de gauge não massivos, o que não condiz com os experimentos, já que os bósons da interação eletrofraca são massivos, com $M_{Z}=91.1876 \pm 0.0021 \mathrm{GeV}$ e $M_{W}=80.385 \pm 0.015 \mathrm{GeV}$ [7]. É necessário descrever um modelo em que a simetria é espontaneamente quebrada, ou pode-se dizer escondida, a fim de gerar tais termos de massa.

Será descrita a quebra espontânea de simetria, sendo o primeiro exemplo descrito por uma simetria discreta. Para um campo escalar real $\phi$, a Lagrangiana é descrita como:

$$
\mathcal{L}=\frac{1}{2}\left(\partial_{\mu} \phi\right)\left(\partial^{\mu} \phi\right)-V(\phi)
$$

Com $V(\phi)$ potencial funcional do campo $\phi$ tal que $V(\phi)=V(-\phi)$, de modo que $\mathcal{L}$ seja invariante sob a simetria $\phi \rightarrow-\phi$. O potencial renormalizável mais simples será escolhido de modo que ele terá a forma de um termo de massa mais um termo de interção $\phi^{4}$. Nota-se que termos de ordem ímpares não serão invariantes sob tal simetria, enquanto termos de ordem superior $\phi^{4}$ serão não renormalizáveis.

$$
V(\phi)=\frac{1}{2} m^{2} \phi^{2}+\frac{\lambda}{4} \phi^{4}
$$

Supondo que a massa é positiva, ao minimizar o potencial acima (1.1.1.2) obtém-se apenas um valor mínimo para $\langle\phi\rangle_{0}$, chamado valor esperado de vácuo (VEV):

$$
\langle\phi\rangle_{0}=0 .
$$

Entretanto, substituí-se o valor do termo de massa da seguinte maneira : $m^{2} \rightarrow-\mu^{2}$,

$$
V(\phi)=-\frac{1}{2} \mu^{2} \phi^{2}+\frac{\lambda}{4} \phi^{4} .
$$

Deste modo, o potencial terá dois VEV:

$$
\langle\phi\rangle_{0}= \pm \sqrt{\frac{-\mu^{2}}{\lambda}} \equiv \pm v
$$



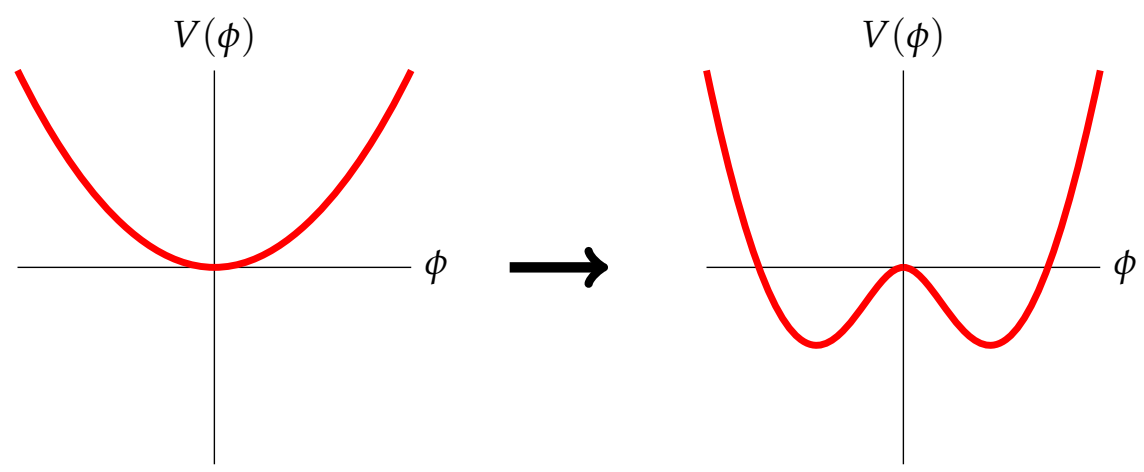

Figura 1.1: Exemplo de quebra espontânea de simetria. Potencial do campo escalar $V(\phi)$.

Ou seja, são criados dois estados de menor energia degenerados.

A invariância sob a simetria $\phi \rightarrow-\phi$ assegura que a escolha de vácuo seja livre.

Logo, escolhendo

$$
\langle\phi\rangle_{0}=+v,
$$

é possível expandir o potencial em torno do mínimo. Assim o campo escalar é redefinido perto do VEV:

$$
\phi^{\prime}=\phi-\langle\phi\rangle_{0}=\phi-v,
$$

com

$$
\left\langle\phi^{\prime}\right\rangle_{0}=0 \text {. }
$$

Substituindo campo $\phi \rightarrow \phi^{\prime}$, expandido em torno do VEV, a nova Lagrangiana será dada por:

$$
\mathcal{L}=\frac{1}{2}\left(\partial_{\mu} \phi^{\prime}\right)\left(\partial^{\mu} \phi^{\prime}\right)-\mu^{2}\left(\phi^{\prime 2}+\frac{\phi^{\prime 3}}{v}+\frac{\phi^{\prime 4}}{4 v^{2}}-\frac{v^{2}}{4}\right) .
$$

Observa-se que são obtidos termos de interação cúbico e quártico, além de uma constante irrelevante. Estes não são invariantes sob a simetria inicial do problema. Diz-se que a simetria foi espontaneamente quebrada ou está escondida.

\section{Bósons de Nambu Goldstone}

No caso em que há uma simetria global contínua é aplicado o Teorema de Goldstone. Ele afirma que deve haver uma partícula escalar não massiva para cada simetria contínua espontânemente quebrada. Consequentemente, são gerados chamados Bósons de Nambu Goldstone (bNG) não massivos. Será descrito um sistema físico com simetria U(1) global a fim de ilustrar o teorema, assim como descrito na referência [8]. Sendo a Lagrangiana descrita para campos escalares complexos: 


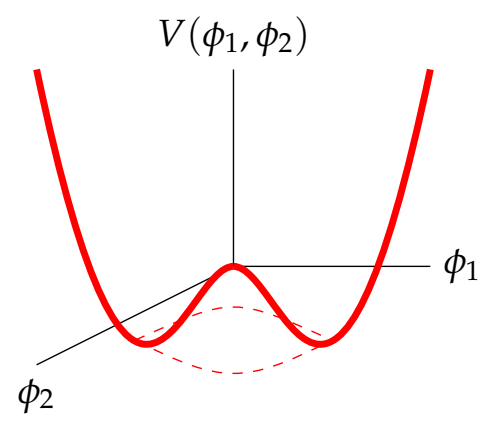

Figura 1.2: Potencial para um campo escalar complexo após quebra espontânea de simetria.

$$
\mathcal{L}=\left(\partial_{\mu} \phi\right)^{*}\left(\partial^{\mu} \phi\right)-\mu^{2} \phi^{*} \phi-\lambda\left(\phi^{*} \phi\right)^{2},
$$

com $\mathcal{L}$ invariante sob simetria $U(1)$, ou seja, $\phi \rightarrow e^{i \alpha} \phi$. Novamente, o potencial é tal que $\lambda>0, \mu^{2}<0$. Além disso, é possível escrever o campo complexo em termos dos seus graus de liberdade, $\operatorname{com} \phi_{1,2}$ reais.

$$
\begin{aligned}
\phi & =\phi_{1}+i \phi_{2} . \\
\phi^{*} & =\phi_{1}-i \phi_{2} .
\end{aligned}
$$

Logo, o potencial é dado por:

$$
V\left(\phi_{1}, \phi_{2}\right)=-\frac{1}{2} \mu^{2}\left(\phi_{1}^{2}+\phi_{2}^{2}\right)-\frac{1}{4} \lambda\left(\phi_{1}^{2}+\phi_{2}^{2}\right)^{2} .
$$

Ao minimizá-lo, verifica-se que os VEVs são invariantes sob uma transformação $U(1)$ equivalente a uma rotação no plano complexo por um ângulo $\alpha$, como demonstrado na figura:

Resumindo, pode-se reescrever os VEVs como:

$$
\begin{gathered}
\phi_{1}^{2}+\phi_{2}^{2}=v, \\
v^{2}=-\frac{\mu^{2}}{\lambda} .
\end{gathered}
$$

Como há liberdade na escolha do VEV, ela será feita de modo que o VEV obtenha um valor real, com $\phi_{1}=v, \phi_{2}=0$. Expandindo o potencial em torno deste ponto:

$$
\phi(x)=\sqrt{\frac{1}{2}}[v+\eta(x)+i \xi(x)] .
$$


A Lagrangiana será descrita como em termos dos campos escalares $\eta$ e $\pi$ :

$$
\mathcal{L}=\frac{1}{2}\left(\partial_{\mu} \xi\right)^{2}+\frac{1}{2}\left(\partial_{\mu} \eta\right)^{2}+\mu^{2} \eta+\text { termos de interação. }
$$

Observa-se que a componente real, ou seja, o campo $\eta$ possui um termo massa. Por outro lado, como previsto no Teorema de Goldstone, $\xi$ permanece não massivo, com apenas termos cinético e de interação. Logo, a escolha de vácuo é feita tal que a componente na direção do vácuo é massiva, enquanto a ortogonal não obtém termos de massa. O campo $\xi$ corresponde ao chamado bóson de Nambu Goldstone (bNG) da teoria.

Foi apresentada a quebra de simetria $U(1)$ global. No entanto, o MP é uma teoria de gauge local que utiliza o mecanismo de Higgs como quebra espontânea da simetria $S U(2)_{L} \times U(1)_{Y} \rightarrow U(1)_{E M}$. As teorias de gauge não apresentam $\mathrm{bNG}$, no MP eletrofraco eles serão absorvidos pelas componentes longitudinais dos bósons de gauge. O modelo será discutido a seguir nas seções que expõe o mecanismo de Higgs e as teorias eletrofracas.

\subsubsection{Mecanismo de Higgs}

O Mecanismo de Higgs consiste em uma quebra espontânea de simetria de gauge, a qual dá origem aos termos de massas dos bósons de gauge, além de obter um campo escalar remanescente, o chamado Bóson de Higgs. O modelo foi proposto em 1964 por Robert Brout, Francois Englert [9]; Peter Higgs [10]; Gerald Guralnik, C. R. Hagen, Tom Kibble [11], tanto o caso abeliano quanto sua generalização para o nãoabeliano. Todavia, é interessante mencionar que o mecanismo é análogo à teoria de supercondutores, desenvolvida anteriormente por Landau Ginzburg para explicar o efeito Meissner, como pode ser estudado na referência [12].

A fim de exemplificar o Mecanismo de de Higgs, supõe-se o modelo para campo escalar com a Lagraniana invariante sob simetria $\mathrm{U}(1)$ de gauge.

$$
\mathcal{L}=-\frac{1}{4} F_{\mu \nu} F^{\mu \nu}+D_{\mu} \phi D^{\mu} \phi-V(\phi)
$$

A derivada covariante é descrita por:

$$
D_{\mu}=\partial_{\mu}+i e A_{\mu}
$$

onde $\phi, \phi^{*}$ representam um campo escalar complexo que pode ser escrito a partir de seus dois graus de liberdade:

$$
\phi=\frac{1}{\sqrt{2}}\left(\phi_{1} \pm i \phi_{2}\right) .
$$

Sendo a invariância local sob U(1) dada pelas transformações

$$
\begin{array}{r}
\phi \rightarrow e^{i \alpha(x)} \phi, \\
A_{\mu} \rightarrow A_{\mu}(x)-\frac{1}{e} \partial_{\mu} \alpha(x) .
\end{array}
$$


Onde o potencial é descrito por:

$$
V(\phi)=-\mu^{2} \phi^{*} \phi+\frac{\lambda}{2}\left(\phi^{*} \phi\right)^{2} .
$$

Ao minimizar esse potencial, observa-se que o campo escalar adquire um VEV para $\mu^{2}>0$, e a simetria U(1) é quebrada expontaneamente. Então, primeiro deve-se redefinir o vácuo da teoria para descrever esse processo:

$$
\langle\phi\rangle_{0}=\sqrt{\frac{\mu^{2}}{\lambda}} .
$$

Ao expandir o campo escalar complexo ao redor do seu VEV, é obtido:

$$
\phi(x)=\langle\phi\rangle_{0}+\frac{1}{\sqrt{2}}\left(\phi_{1}(x)+i \phi_{2}(x)\right) .
$$

Substitui-se o campo expandido em torno do VEV no potencial:

$$
V(\phi)=-\frac{1}{2 \lambda} \mu^{4}+\frac{1}{2} 2 \mu^{2} \phi_{1}^{2}+O\left(\phi_{i}^{3}\right) .
$$

Finalmente, para de obter os termos de massa gerados a partir da quebra espontânea de simetria, o termo cinético da Lagrangiana é descrito redefinindo o campo escalar complexo em torno do mínimo (1.1.2.8).

$$
\begin{aligned}
\left|D_{\mu} \phi\right|^{2}= & \frac{1}{2}\left(\partial_{\mu} \phi_{1}\right)^{2}+\frac{1}{2}\left(\partial_{\mu} \phi_{2}\right)^{2}+\sqrt{2} e \phi_{0} \times A_{\mu} \partial^{\mu} \phi_{2}+\mu^{2} \phi_{1}^{2}+ \\
& +e^{2} \phi_{0}^{2} A_{\mu} A^{\mu}+\text { termos de interação. }
\end{aligned}
$$

Obseva-se que um grau de liberdade do campo $\phi$ não é massivo, enquanto o outro obtém massa. Ou seja, o campo $\phi_{1}$ adquire massa $m=\sqrt{2} \mu$, enquanto $\phi_{2}$ permanece não massivo. Por outro lado, nota-se que o fóton aparentemente adquire um termo de massa em (1.1.2.10) $\operatorname{com} m_{A}^{2}=2 e^{2} \phi_{0}^{2}$, tal que:

$$
\Delta \mathcal{L}=\frac{1}{2} m_{A}^{2} A_{\mu} A^{\mu}
$$

Além disso, elege-se o gauge unitário, já que existe um termo indesejável de mistura entre o bóson de gauge e a derivada do campo $\phi_{2}$. Nesse caso o campo $\phi_{2}$ é eliminado inteiramente, inclusive seu termo cinético não existe nessa escolha de gauge. Para respeitar a simetria de gauge $\mathrm{U}(1)$, são selecionados $\alpha(x)$ na transformação de gauge (1.1.2.4) tal que os campos $\phi(x)$ sejam reais em todos os pontos. Deste modo, é possível eliminar os termos indesejáveis e obter a Lagrangiana com os devidos termos de massa dos bósons de gauge:

$$
\mathcal{L}=-\frac{1}{4} F_{\mu v} F^{\mu v}+\partial_{\mu} \phi \partial^{\mu} \phi+e^{2} \phi_{0}^{2} A_{\mu} A^{\mu}+\mu^{2} \phi^{2}+\text { termos de interação }
$$

Resumindo, o modelo inicialmente descrevia 2 campos escalares complexos $\phi, \phi^{*}$, com dois graus de liberdade, além de duas helicidades do boson de gauge $A_{\mu}$ não 
massivo. Após a quebra espontânea de simetria, surgiram um escalar e três estados de helicidade do $A_{\mu}$ massivo. Portanto, pode se dizer que o campo de gauge "absorveu"o BNG, e o campo remanescente $\phi$ é chamado de bóson de Higgs.

\subsubsection{Teoria de interações eletrofracas}

Enfim, será apresentado o modelo que descreve as teorias eletrofracas. A teoria foi proposta por S. L. Glashow em 1961 [13], S. Weinberg em 1967 [14] e A. Salam. O modelo eletrofraco consiste em uma teoria de gauge invariante sob simetria $S U(2)_{L} \times$ $U(1)_{Y}$. Através do mecanismo de Higgs, esta simetria gera três bósons vetoriais massivos $W_{\mu}^{ \pm}$e $Z_{\mu}^{0}$, além de um campo vetorial não massivo, o fóton $A_{\mu}$. Logo, a Lagrangiana $\mathcal{L}_{E F}$ invariante sob $S U(2)_{L} \times U(1)_{Y}$ é escrita por:

$$
\mathcal{L}_{E F}=\left(D^{\mu} \phi\right)^{\dagger}\left(D_{\mu} \phi\right)-\frac{1}{4} A_{\mu \nu}^{a} A^{a \mu \nu}-\frac{1}{4} B_{\mu v} B^{\mu \nu}-V\left(\phi^{\dagger} \phi\right) .
$$

Denotando $\phi$ como um dubleto escalar complexo. Além disso, a derivada covariante é descrita abaixo. Os bósons de gauge correspondente às simetrias $S U(2)_{L}$ e $U(1)_{Y}$ são respectivamente $A_{\mu}$ e $B_{\mu}$, com g e g' seus acoplamentos.

$$
D_{\mu} \phi=\left(\partial_{\mu}-i g A_{\mu}^{a} \tau^{a}-i Y g^{\prime} B_{\mu}\right) \phi .
$$

Novamente o potencial será da forma:

$$
V\left(\phi^{\dagger} \phi\right)=-m^{2}\left(\phi^{\dagger} \phi\right)+\lambda\left(\phi^{\dagger} \phi\right)^{2} .
$$

O campo $\phi$ é uma representação de um dubleto complexo de $S U(2)_{L}$, composto por 4 graus de liberdade, que correspondem a escalares reais.

$$
\phi=\left(\begin{array}{c}
\phi^{+} \\
\phi^{0}
\end{array}\right)
$$

Dada a simetria $S U(2) \times U(1)$, os campos escalares se transformam como de acordo com:

$$
\phi \rightarrow e^{i \alpha^{a} \tau^{a}} e^{i \beta / 2} \phi,
$$

com a hipercarga de $Y_{\phi}=\frac{1}{2}$ correspondente à simetria de gauge $\mathrm{U}(1)$. Os geradores do grupo $S U(2)_{L}$ são dados por $\tau^{a}=\frac{\sigma^{a}}{2}$, tal que $\sigma^{a} \operatorname{com} a=1,2,3$ são as matrizes de Pauli. O VEV é dado por $v \approx 246 \mathrm{GeV}$, escolhido como:

$$
\langle\phi\rangle=\frac{1}{\sqrt{2}}\left(\begin{array}{l}
0 \\
v
\end{array}\right) .
$$

Portanto, deve-se expandir o termo cinético da Lagrangiana ao redor do VEV (1.1.3.6) para obter as massas dos bósons de gauge. Logo, o termo cinético:

$$
\Delta \mathcal{L}=\left(D_{\mu} \phi\right)^{\dagger}\left(D^{\mu} \phi\right)
$$


Substituindo o campo $\phi$ pelo VEV, obtemos a Lagrangiana:

$$
\Delta \mathcal{L}=\frac{1}{2}\left(\begin{array}{ll}
0 & v
\end{array}\right)\left(g A_{\mu}^{a} \tau^{a}+\frac{1}{2} g^{\prime} B_{\mu}\right)\left(g A^{b \mu} \tau^{b}+\frac{1}{2} g^{\prime} B^{\mu}\right)\left(\begin{array}{l}
0 \\
v
\end{array}\right)+\ldots
$$

Os termos de massas podem ser escritos em função dos bósons de gauge $A_{\mu}^{a}$ e $B_{\mu}$.

$$
\Delta \mathcal{L}_{\text {massa }}=\frac{1}{2} \frac{v^{2}}{4}\left[g^{2}\left(A_{\mu}^{1}\right)^{2}+g^{2}\left(A_{\mu}^{2}\right)^{2}+\left(-g A_{\mu}^{3}+g^{\prime} B_{\mu}\right)^{2}\right],
$$

de onde se identificam os quatro bósons de interação eletrofraca. Nota-se que os termos em $A_{\mu}^{1,2}$ corresponderão aos bósons carregados $W_{\mu}^{ \pm}$:

$$
\begin{array}{r}
W_{\mu}^{ \pm}=\frac{1}{\sqrt{2}}\left(A_{\mu}^{1} \mp i A_{\mu}^{2}\right), \\
m_{W}=g \frac{v}{2} .
\end{array}
$$

A matriz de massas será dada por

$$
\frac{v^{2}}{4}\left(\begin{array}{cc}
g^{2} & -g g^{\prime} \\
-g g^{\prime} & g^{2}
\end{array}\right)
$$

tem autovalor nulo e outro diferente de zero. No entanto, há mistura entre $A_{\mu}^{3}$ e $B_{\mu}$, que dão origem aos bósons neutros, tanto o massivo $Z_{\mu}^{0}$, assim como seu termo ortogonal não massivo que é identificado como o fóton $\gamma$

$$
\begin{array}{r}
Z_{\mu}^{0}=\frac{1}{\sqrt{g^{2}+g^{\prime 2}}}\left(g A_{\mu}^{3}-g^{\prime} B_{\mu}\right), \\
m_{Z}=\sqrt{g^{2}+g^{\prime 2}} \frac{v}{2} . \\
A_{\mu}=\frac{1}{\sqrt{g^{2}+g^{\prime 2}}}\left(g^{\prime} A_{\mu}^{3}+g B_{\mu}\right), \\
m_{\gamma}=0 .
\end{array}
$$

Pode-se fazer uma transformação unitária para descrever a mudança de base dos campos de gauge $A_{\mu}^{3}$ e $B_{\mu}$ em função dos bósons físicos neutros $A_{\mu}$ e $Z_{\mu}^{0}$. Deste modo, a matriz de rotação descrita pelo ângulo de Weinberg pode ser definida por relações como

$$
\tan \theta=\frac{g^{\prime}}{g}
$$

com a transformação de base entre os bósons de gauge $A_{\mu}^{3}, B_{\mu}$ e os observáveis $Z_{\mu}^{0}$ $A_{\mu}$ sendo uma rotação em $\theta$ :

$$
\left(\begin{array}{l}
Z_{\mu}^{0} \\
A_{\mu}
\end{array}\right)=\left(\begin{array}{cc}
\cos \theta & -\operatorname{sen} \theta \\
\operatorname{sen} \theta & \cos \theta
\end{array}\right)\left(\begin{array}{l}
A_{\mu}^{3} \\
B_{\mu}
\end{array}\right)
$$




\subsubsection{Férmions no MP}

No Modelo Padrão, tanto os léptons quanto os quarks de mão esquerda são descritos por um dubleto de $S U(2)_{L}$.

$$
L_{L}=\left(\begin{array}{c}
v_{e_{L}} \\
e_{L}^{-}
\end{array}\right), Q_{L}=\left(\begin{array}{c}
u_{L} \\
d_{L}
\end{array}\right)
$$

Lembrando que a projeção dos spinors fermiônicos para as helicidades são dadas pelas matrizes $\gamma^{5}$

$$
\psi_{R, L}=\frac{1}{2}\left(1 \pm \gamma^{5}\right) \psi
$$

A relação de Gell-Mann-Nishijima para a carga elétrica define a hipercarga Y, sendo $T^{3}$ projeção de isospin-fraco

$$
Q=T^{3}+Y \text {. }
$$

Os férmions de mão direita são representados pelos singletos $u_{R}$ e $d_{R}$. Entretanto, é imporante ressaltar que o neutrino de mão direita tem hipercarga nula, assim como sua carga, consequentemente $T_{v}^{3}=0$. O que significa que neutrinos de mão direita não se acoplam com bósons do MP. Logo, para os léptons existe apenas o singleto de mão direita $e_{R}$. Estas partículas têm a carga definida apenas pela simetria $U(1)_{Y}$,

$$
Q=Y
$$

\subsubsection{Termo de Yukawa das massas dos férmions}

É certo que os termos de massa para os férmions violam explicitamente a simetria de gauge. Por essa razão, o operador que gera as massas deles, invariante de gauge e renormalizável é dado pelo termo de Yukawa. Para os léptons ele é descrito por:

$$
\begin{aligned}
\Delta \mathcal{L}_{l} & =-\lambda_{l} \bar{L}_{L} \phi e_{R} \\
m_{e} & =\frac{1}{\sqrt{2}} \lambda_{e} v .
\end{aligned}
$$

Enquanto os quarks:

$$
\Delta \mathcal{L}_{q}=-\lambda_{q}^{i j} \bar{Q}_{L} \phi d_{R}-\lambda_{u}^{i j} \epsilon^{a b} \bar{Q}_{L} a \phi_{b}^{\dagger} u_{R}+\text { h.c., }
$$

onde $\lambda_{q}^{i j}$ são matrizes de acoplamento de Yukawa. Ao expandir este termo ao redor do mínimo

$$
\phi=\left(\begin{array}{c}
0 \\
\frac{v+h(x)}{\sqrt{2}}
\end{array}\right)
$$

concluí-se que a matriz de massas dos férmions é dada por: 


$$
\begin{aligned}
& M_{d}^{i j}=\frac{1}{\sqrt{2}} \lambda_{d}^{i j} v, \\
& M_{u}^{i j}=\frac{1}{\sqrt{2}} \lambda_{u}^{i j} v .
\end{aligned}
$$

Entretanto, a matriz de massa respectiva às três famílias de quarks não é diagonal, nem real. Sendo os quarks representados pelos tripletos de sabor :

$$
u_{L}^{i}=\left(\begin{array}{c}
u_{L} \\
c_{L} \\
t_{L}
\end{array}\right), d_{L}^{i}=\left(\begin{array}{c}
d_{L} \\
s_{L} \\
b_{L}
\end{array}\right)
$$

É necessário realizar uma transformação unitária na base que representam as três famílias de quarks, para que estes deem lugar ao autoestados de massa:

$$
u_{L}^{i}=U_{u}^{i j} u_{L^{\prime}}^{\prime j} d_{L}^{i}=U_{d}^{i j} d_{L^{\prime}}^{j}
$$

Entretanto, as correntes carregadas terão efeito imediato ao serem descritas a partir dos autoestados de massa. Eles darão lugar a interações com mistura entre as famílias de quarks.

$$
J_{W}^{\mu+}=\frac{1}{\sqrt{2}} \bar{u}_{L}^{i} \gamma^{\mu} d_{L}^{i} \rightarrow \frac{1}{\sqrt{2}} \bar{u}_{L}^{i} \gamma^{\mu}\left(U_{u}^{\dagger} U_{d}\right) d_{L}^{i}
$$

Por definição, a matriz Cabibbo-Kobayashi-Maskawa (CKM) de mistura dos quarks é dada por:

$$
V_{i j} \equiv\left(U_{u}^{\dagger} U_{d}\right) .
$$

A matriz CKM depende de 3 ângulos independentes e uma fase complexa. Ela foi bem medida experimentalmente, sendo aproximadamente 1 na diagonal e os demais valores de mistura entre as famílias são menores [15].

Analogamente, existe matriz de mistura dos leptons, chamada matriz PMNS (PontecorvoMaki-Nakagawa-Sakata) [16]. Ela pode ser estudada nas referências [17] e [18] . Nesse trabalho será utilizado o conceito de mistura dos quarks, por isso foi discutida em mais detalhe apenas a matriz CKM, já que a fenomenologia se concentra no decaimento de modos excitados da Teoria Quiver em quarks do MP. A análise do setor leptônico está em preparação no grupo de pesquisa [19].

\subsubsection{Testes experimentais}

O MP da Física de Partículas é uma teoria bem estabelecida e está de acordo com os testes experimentais. Foram observados os bósons de interação eletrofracos $W^{ \pm}$e $Z^{0}$ no LEP (Large Electron-Positron), no CERN, além da confirmação de três famílias de quarks e leptons em diversos experimentos [20] [21]. Recentemente foi observado 


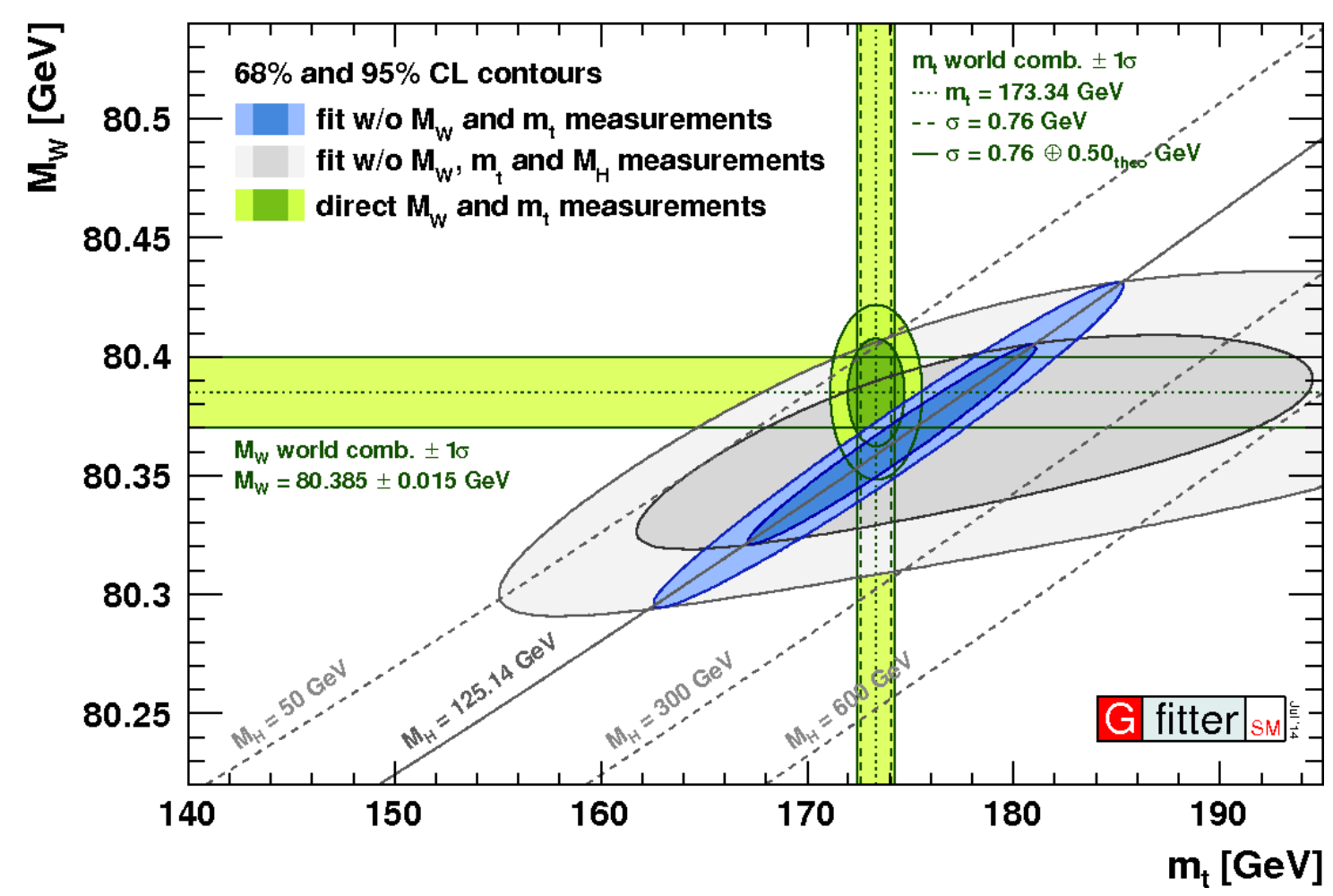

Figura 1.3: Medições diretas e cálculo com correções às massas do bóson $W$ e quark top. Observa-se que as faixas verdes são as medições diretas das massas $m_{W}$ e $m_{t}$ a $1 \sigma$ na média mundial. Enquanto a região cinza e azul são os cálculos indiretos dessas massas. A cinza exclui medições à massa do Higgs, entretanto a zona azul é mais estreita com $m_{H}=125 \mathrm{GeV}$. A intersecção dessas regiões demonstra que o MP é sensível a correções radiativas, e válido até a escala TeV. Figura retirada da referência [24]

no LHC uma ressonância a $125 \mathrm{GeV}$ correspondente ao bóson de Higgs, o que completa o MP, publicado pelas colaborações ATLAS e CMS nas referências [22] e [23]. É importante ressaltar que todos os acoplamentos dos férmions com bósons de gauge do MP foram medidos com grande precisão. No entanto, os acoplamentos do Higgs com bósons de gauge e férmions ainda não foram completamente medidos, sendo necessário aguardar a próxima fase do LHC para futuros resultados que definirão as interações do novo bóson.

Medidas de precisão podem ser usadas a fim de testar o MP além da primeira ordem em teoria de perturbação. Por exemplo, as medidas eletrofracas de precisão são sensíveis às massas do top e Higgs $m_{t}$ e $m_{H}$ no loop dos propagadores dos bósons de gauge, assim como é demonstrado na figura 1.3.

A figura 1.3 demonstra medições diretas e indiretas da massa do $\mathrm{W} m_{W}$ e do quark top $m_{t}$, retirado da referência [24]. As faixas verdes são os contornos a $68 \%$ e $95 \%$ no 
nível de confiança obtidos para as massas das partículas em questão a $1 \sigma$ na média mundial. A dependência de $\Delta m_{t}$ é quadrática, enquanto $\Delta m_{H}$ é só logarítimica. A diferença entre as correções radiativas à massa do top e Higgs fica clara já que a faixa vertical que demonstra o cálculo a um loop de $m_{t}$ é muito mais precisa do que as linhas diagonais de $m_{H}$. Por outro lado, as regiões cinza e azul demonstram cálculos indiretos para $m_{W}$ e $m_{t}$ considerando correções radiativas. Sendo que a região maior não considera as medições à massa do Higgs, o qual varia de 50 a $600 \mathrm{GeV}$. No entanto, a região mais estreita azul fixa $m_{H}=125 \mathrm{GeV}$. A descoberta de um Higgs leve implica no grande sucesso do MP. Observa-se que a elipse a $125 \mathrm{GeV}$ coincide com a região onde $m_{t}^{\text {exp }}$ e $m_{W}^{\text {exp }}$ são medidos. Portanto, o modelo é sensível à cálculos a 1 loop, já que a intersecção das zonas demonstradas na figura implicam que deve haver uma solução. Logo, observa-se que o teste ao $\mathrm{MP}$ o valida até a escala $\mathrm{TeV}$ com grande precisão.

Medidas de precisão eletrofracas também podem ser demonstradas a partir dos parâmetros de Peskin-Tekeuchi S, T e U. Onde S é medido a partir de auto energias no cálculo de $M_{Z}$, U obtém-se a partir de $M_{W}$ e T de $\gamma_{Z}$. Eles são utilizados também para definir a fenomenologia em Teorias Quiver. Há diversos experimentos que testam violação de sabor que devem ser considerados. Eles também impõe vínculos importantes em teorias além do MP, por isso serão discutidos no capítulo 3.

Além disso, os testes eletrofracos de precisão no MP são feitos através de medições dos observáveis como a massa do $Z$, constante de Fermi $G_{F}$ e de estrutura fina $\alpha$. Logo, é possível calcular correções radiativas a partir dessas medições, o que impõe restrições nos demais parâmetros físicos. Os testes são feitos a partir de medições precisas nos decaimentos $\mathrm{W}$ e $\mathrm{Z}$, e das assimetrias right-left e assimetria foward-backward.

$$
A_{F B} \equiv \frac{\sigma_{F}-\sigma_{B}}{\sigma_{F}+\sigma_{B}} .
$$

É importante mencionar que correções radiativas também impõem restrições em modelos além do MP. O MP da física de partículas é uma teoria muito bem estabelecida. Violação de unitariedade previa um escalar massivo leve, como de fato encontrado no LHC, o chamado bóson de Higgs com massa $125 \mathrm{GeV}$. Com isto, o MP torna-se completo e comprovado ser uma teoria válida a esta escala de energia. No entanto, este modelo deixa ainda algumas questões a serem respondidas.

\subsection{Física Além do Modelo Padrão}

Existem motivações para procura de nova física na escala TeV. Diversas questões permanecem em aberto, no entanto o problema de hierarquia de Gauge leva a crer que pode ser descoberta nova física em experimentos no LHC. Além disso, também será tratado o problema de hierarquia das massas dos férmions. Nos capítulos 2 e 3 será discutido como Teorias Quiver são candidatas a resolver essas questões.

O MP não abrange todos os temas na Física de Partículas, tais como a descrição de matéria escura, constante cosmológica ou mesmo da gravidade quântica. Além disso, 


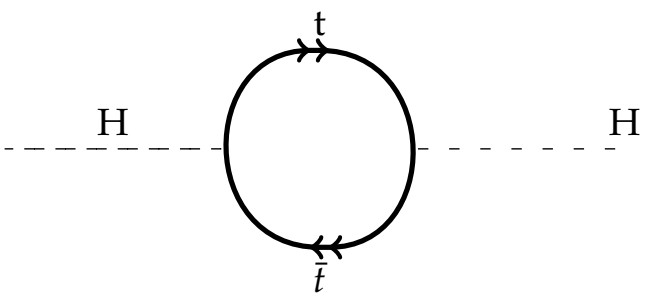

Figura 1.4: Diagrama a um loop com maior contribuição à correção radiativa de $m_{H}^{2}$.

ele descreve apenas neutrinos com massa nula, enquanto é certo que eles devem ter uma massa muito pequena, inferior a $2 \mathrm{eV}$ [20], pois a oscilação de neutrinos observada indica que estes devem ter massa diferente de zero. Outras questões como a assimetria matéria-antimatéria ou o problema da violação CP nas interações fortes também necessitam maior compreensão.

O problema da hierarquia das massas dos férmions será analisado nesse trabalho. Apesar de o MP explicar a origem das massas dos férmions, resta a pergunta dos acoplamentos de Yukawa terem valores tão diferentes. Para o elétron, acoplamento é mais fraco $y_{e} \sim 10^{-6}$ com $m_{e}=0.511 \mathrm{MeV}$ [20]. Enquanto, para o quark top $y_{t} \sim 1$ com $m_{t}=173 \mathrm{GeV}$ [21]. Teorias além do MP são propostas de modo que é gerada a hierarquia das massas dos férmions naturalmente.

\subsubsection{Problema de hierarquia de gauge}

$\mathrm{O}$ MP da física de partículas descreve muito bem as interações na escala eletrofraca $(\simeq 100 \mathrm{GeV})$. No entanto, não se sabe qual sua validade, podendo chegar até ordem da massa de Planck onde as interações gravitacionais devem ser consideradas. É preciso quantizar a gravitação acima dessa escala, já que a teoria torna-se fortemente acoplada, ou seja, interações com grávitons têm contribuições muito maiores do que as geradas por bósons do MP nessa escala de energia. Por essa razão, é dito que a teoria tem um corte Ultra violeta (UV) muito alto, no máximo a escala de Planck $\left(\simeq 10^{19} \mathrm{GeV}\right)$. Essa discrepância implica que deve haver um ajuste fino na teoria efetiva. Logo, são propostas teorias além do MP, candidatas a resolver o problema de hierarquia gerando nova física na escala $\mathrm{TeV}$.

Com a descoberta de um Higgs leve, $m_{H}=125 \mathrm{GeV}$, foi possível calcular o acoplamento $\lambda$. Já que $m_{H}=\sqrt{2 \lambda v}$, foi medido $\lambda \simeq 0.13$. Portanto, o acoplamento é pequeno o suficiente para que as correções radiativas descrevam o MP perturbativamente até a escala de Planck. Ao calcular as correções radiativas à massa do Higgs:

$$
m^{2}=m_{n u a}^{2}+\delta m_{\text {quantica }}^{2}
$$

observa-se que a massa do Higgs é descrita pela soma das correções quânticas $\delta m^{2}$ à massa nua, a qual representa um contratermo, e como tal tem origem no UV. 


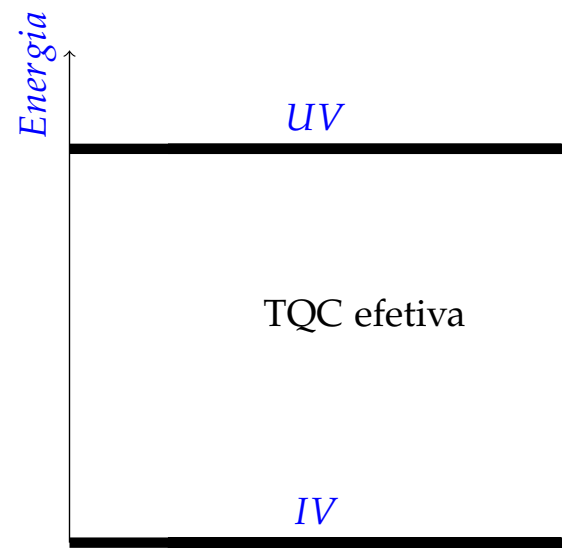

Figura 1.5: Ilustração exemplificando a faixa de energia onde o MP é válido.

O diagrama a um loop que mais contribui às correções à massa do Higgs é descrito pela criação do par virtual de quarks top, já que o acoplamento de yukawa deles é maior que os demais férmions. A contribuição do diagrama acima resulta na correção à massa do Higgs a um loop dada por:

$$
\delta m_{\text {quantica }}^{2}=(-) \frac{3 \lambda_{t}^{2}}{8 \pi^{2}} \Lambda_{\text {top }}^{2}+\ldots
$$

Onde $\Lambda_{\text {top }}$ é o maior valor que o momento do quark top pode ter antes do MP deixar de valer, o qual pode ser dado até a escala de Planck. Supõe-se que as partículas virtuais tenham grandes momentos $P_{t}$. Ao dividir as escalas de momentos em camadas, usando a renormalização segundo Wilson, conclui-se que em uma dada camada $\delta m_{H}^{2} \sim O\left(P_{t}^{2}\right)$. Como as correções ao campo escalar são somadas quadráticamente à massa nua, o ajuste fino que deve ser feito na teoria é muito maior do que os observados nos demais parâmetros físicos. A física no infravermelho (IV) terá uma diferença ao UV de 1 parte em $10^{34}$ ao considerar a razão entre $M_{\text {Planck }}^{2}$ e $M_{\text {eletrofraco. }}^{2}$. Ou seja, a condição IV ajustada com UV é muito grande. Ressaltando que, nessa dissertação, será denotado as abreviações para os termos infravermelho e ultravioleta como IV e UV.

Para evitar esse problema, acredita-se que exista nova física na escala TeV. As soluções incluem propostas com simetrias adicionais ou com descrições do Higgs como bóson de Nambu Goldstone. Foram apresentadas na literatura diversas teorias para resolver o problema de hierarquia de Gauge. Os principais modelos candidatos à solucionar tal problema são Supersimetria, Little Higgs, ou Teorias com Dimensões Extras. Nesse trabalho são apresentadas as Teorias Quiver como canditas a resolver o problema de hierarquia, nas quais o Higgs é um pseudo bóson de Nambu Goldstone. 


\subsubsection{Teorias Quiver como solução ao problema de hierarquia}

Serão apresentadas as Teorias Quiver como solução ao problema de hierarquia. Elas são modelos que consistem em uma cadeia de grupos de gauge conectados por campos de ligação, desenvolvidos para de gerar a hierarquia entre as escalas eletrofraca e UV. A quebra da simetria eletrofraca gera tanto uma torre de modos excitados dos bósons de gauge, como a escala de massas dos férmions. Logo, a hierarquia de massas dos férmions é gerada naturalmente. Será apresentado no capítulo 3 a descrição do bóson de Higgs no Quiver como um pseudo bóson de Nambu Goldstone. Esse mecanismo mantém os graus de liberdade do Higgs no sítio IV, o que é suficiente para resolver o problema de hierarquia, assim como em teorias com dimensões extras.

Estas teorias podem ser obtidas a partir de uma desconstrução dimensional de teorias com uma dimensão extra compacta em um cenário $A d S_{5}$. Sendo uma alternativa quadridimensional às teorias com dimensões extras, já que fazem parte da classe de modelos que descrevem $A d S_{5}$ no limite do contínuo [25]. Entretanto, Teorias Quiver têm uma fenomenologia distinta longe deste limite.

Esse trabalho visa a fenomenologia de Teorias Quiver com poucos sítios, obtendo vínculos com dados acumulados pelo LHC [1]. O que será apresentado em detalhes no capítulo 4. 


\section{Teorias Quiver}

Nesse capítulo são apresentadas as chamadas Teorias Quiver. Elas são candidatas à solução do problema de hierarquia, sendo que essa resolução é tratada no capítulo 3 ao definir o Higgs como um pseudo bóson de Nambu-Goldstone. O objetivo desse capítulo é descrever aspectos teóricos das Teorias Quiver. Então, a partir desse modelo será feita uma análise fenomenológica, estudada finalmente no capítulo 4 . É importante ressaltar que as Teorias Quiver apresentam uma fenomenologia particular na análise de ressonâncias na escala $\mathrm{TeV}$, as quais poderão ser encontradas futuramente pelo LHC.

Teorias Quiver são modelos puramente quadridimensionais, descritos por N+1 grupos de gauge conectados por campos escalares, chamados campos de ligação. Estes grupos estão localizados em diferentes sítios ligados por modelos sigma, os quais possuem valores esperados de vácuo ordenados entre as escalas Ultra-Violeta e Infra Vermelha, a qual será identificada como a escala eletro fraca. Os campos de ligação são quebrados dinamicamente, gerando uma torre de estados excitados de bósons de gauge. Além disso, ao incluir férmions no modelo, a quebra de simetria em cascata gera naturalmente a hierarquia de massas dos férmions.

Teorias Quiver podem ser obtidas ao desconstruir uma teoria de dimensão extra com métrica Anti de Sitter (AdS). As teorias em AdS podem ser retomadas no limite do contínuo, especificamente para valores grandes de $\mathrm{N}$ e alto valor esperado de vácuo do sítio Ultra-Violeta. É possível construir um dicionário que relaciona as duas teorias, sendo claro que as teorias desconstruidas com muitos sítios se comportam de maneira indistinguivel às teorias em $A d S$. Todavia, para poucos sítios, estes modelos apresentam fenomenologia distinta [25]. Por esta razão, foi escolhido trabalhar com teorias a poucos sítios, longe do contínuo, a fim de gerar um modelo com novos resultados fenomenológicos e mínima violação de sabor. Esses cálculos serão desenvolvidos em maior detalhe nos capítulos 3 e 4 . 


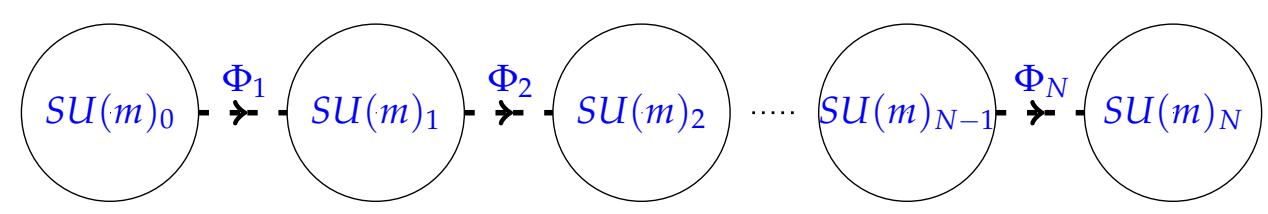

Figura 2.1: Diagrama de quiver para uma teoria com N sítios em uma cadeia linear.

\subsection{Quebra de simetria em Teorias Quiver}

O modelo que descreve as Teorias Quiver é constituído por um produto de $N+1$ grupos de gauge, o que pode ser estudado também nas referências [25] [26] [27]. Estes serão descritos em uma cadeia linear da forma:

$$
G_{0} \times G_{1} \times G_{2} \ldots G_{j} \times G_{j+1} \ldots G_{N},
$$

onde os grupos de gauge são usualmente da forma $G_{j}=S U(m)_{j}, \operatorname{com} j=0,1 \ldots N$, representando grupos de simetria de Yang-Mills. Para cada $G_{j}$ exite um bóson de gauge $A_{\mu, j}^{a}, a=1,2, \ldots, m^{2}-1$. Os grupos de gauge estão unidos por $\mathrm{N}$ campos escalares complexos, chamados de campos de ligação $\Phi_{j}$ que se transformam bifundamentalmente sob $G_{j-1} \times G_{j}$.

A teoria pode ser representada por diagramas chamados moose ou quiver. Em seguida há um exemplo do esquema, onde há uma cadeia linear com $N+1$ círculos, equivalentes aos grupos de gauge. Eles estão ligados por setas horizontais, as quais denotam os campos de ligação $\Phi_{j}$. Logo, os diagramas mostram que os campos de ligação se transformam de maneira fundamental em relação ao grupo à sua esquerda, de onde a seta sai, e antifundamental em relação ao grupo adjacente, para onde a seta aponta.

A ação do modelo descrito pelo diagrama acima é dada por:

$$
S_{\text {Quiver }}=\int d^{4} x\left\{-\sum_{j=0}^{N} \frac{1}{2 g_{j}^{2}} \operatorname{Tr}\left[F_{\mu v}^{j} F^{\mu v, j}\right]+\sum_{j=1}^{N} \operatorname{Tr}\left[\left(D_{\mu} \Phi_{j}\right)^{\dagger}\left(D^{\mu} \Phi_{j}\right)\right]-V\left(\Phi_{j}\right)\right\} .
$$

A ação (2.1.0.1) tem por simetria de gauge $S U(m)^{N}$. Os tensores de intensidade dos campos de gauge $F_{\mu v}$ têm por notação:

$$
\begin{gathered}
F_{\mu v, j}=F_{\mu v, j}^{a} T_{j,}^{a}, \\
a=1,2 \ldots m^{2}-1
\end{gathered}
$$

onde $T_{j}^{a}$ são os geradores dos grupos de gauge $S U(m)_{j}$. Eles obedecem à álgebra que define as constantes de estrutura $f^{a b c}$ :

$$
\left[T_{j}^{a}, T_{j}^{b}\right]=i f_{j}^{a b c} T_{j}^{c}
$$


Enfim, os tensores de intensidade dos campos de gauge podem ser escritos a partir dos bósons de gauge $A_{\mu, j}^{a}$ da seguinte maneira:

$$
F_{\mu v, j}^{a}=\partial_{\mu} A_{v, j}^{a}-\partial_{v} A_{\mu j}^{a}+g f_{j}^{a b c} A_{\mu, j}^{b} A_{v, j}^{c} .
$$

Por outro lado, a derivada covariante na ação (2.1.0.1) é descrita para cada campo de gauge $A_{\mu, j}^{a}$ de cada grupo do quiver $G_{j}$ :

$$
D_{\mu} \Phi_{j}=\partial_{\mu} \Phi_{j}+i g_{j-1} A_{\mu, j-1}^{a} T_{j-1}^{a} \Phi_{j}-i g_{j} \Phi_{j} A_{\mu, j}^{a} T_{j}^{a} .
$$

Pode-se por fim realizar uma soma de modelos sigma para obter a matriz de massa para os bósons de gauge, absorvendo todo os bósons de Nambu Goldstone $\pi_{j}^{a}$. Os valores esperados de vácuo serão obtidos ao supor que os potenciais para os campos de ligação $\Phi_{j}$ quebrem a simetria $G_{j-1} \times G_{j}$ ao grupo diagonal. Logo, o modelo sigma não linear para cada $\Phi_{j}$ é tomado ao reescrevê-lo em termos dos VEV e $\pi_{j}^{a}$, com os geradores quebrados $T^{a}$.

$$
\Phi_{j}=\frac{v_{j}}{\sqrt{2}} e^{i \pi_{j}^{a}(x) T^{a} / v_{j}}
$$

O termo cinético dos campos de ligação devem ser expandidos de acordo com os campos em (2.1.0.4), da seguinte maneira:

$$
\begin{gathered}
\Delta \mathcal{L}_{c i n}=\left(D_{\mu} \Phi_{j}\right)^{\dagger}\left(D^{\mu} \Phi_{j}\right), \\
\Delta \mathcal{L}_{c i n}=\frac{1}{2}\left(\partial_{\mu} \pi_{j}^{a} t_{a, j}\right)\left(\partial^{\mu} \pi_{j}^{a} t_{a, j}\right) \\
+\frac{v_{j}}{2} 2\left(\partial^{\mu} \pi_{j}^{a} t_{a, j}\right)\left(g_{j-1} A_{\mu, j-1}^{a} t_{a, j-1}-g_{j} A_{\mu, j}^{a} t_{a, j}\right) \\
+\frac{v_{j}^{2}}{2}\left(g_{j-1} A_{\mu, j-1}^{a} t_{a, j-1}-g_{j} A_{\mu, j}^{a} t_{a, j}\right)^{2} .
\end{gathered}
$$

O que pode ser simplificado ao termo:

$$
\Delta \mathcal{L}_{c i n}=\frac{1}{2}\left[\partial_{\mu} \pi_{j}^{a} t_{a, j}+v_{j}\left(g_{j-1} A_{\mu, j-1}^{a} t_{a, j-1}-g_{j} A_{\mu, j}^{a} t_{a, j}\right)\right]^{2} .
$$

Por simplicidade, será pressuposto que todos os grupos de gauge sejam identicos:

$$
T_{j}^{a}=T^{a} .
$$

Os acoplamentos de cada sítio também podem ser escritos como um mesmo g:

$$
g_{0}(v)=g_{1}\left(v_{1}\right)=\cdots=g_{j}\left(v_{j}\right)=\cdots=g_{N}\left(v_{N}\right)=g .
$$


Também deve-se fazer uma escolha de gauge para eliminar o termo misto em (2.1.0.6). Considerando a transformação de gauge dos campos $A_{\mu, j}^{a}$ como:

$$
\begin{array}{r}
A_{\mu, j}^{a} T_{a} \rightarrow A_{\mu, j}^{a} T_{a}-\partial_{\mu} \alpha_{j}^{a}(x) T_{a}, \\
A_{\mu, j-1}^{a} T_{a} \rightarrow A_{\mu, j-1}^{a} T_{a} .
\end{array}
$$

Ao escolher o gauge unitário, que pode ser descrito de tal forma:

$$
\alpha_{j}^{a}(x) t_{a}=-\frac{1}{g v} \pi_{j}^{a}(x) .
$$

De modo que o termo cinético dá origem ao termo de massa dos bósons de gauge,

$$
\Delta \mathcal{L}_{\text {cin }}=\frac{v_{j}^{2} g^{2}}{2}\left(A_{\mu, j}^{a} T_{a}-A_{\mu, j-1}^{a} T_{a}\right)^{2},
$$

onde foi usado a relação $\operatorname{Tr}\left[T_{a} T_{b}\right]=\frac{1}{2} \delta_{a b}$. Observa-se que os bósons de NambuGoldstone foram absorvidos pelos campos de gauge massivos, dando origem a uma matriz de massa não diagonal.

$$
\Delta \mathcal{L}_{\text {massa }}=\frac{v_{j}^{2} g^{2}}{2}\left(A_{\mu, j}-A_{\mu, j-1}\right)^{2},
$$

$\operatorname{com} A_{\mu, j} \equiv A_{\mu, j}^{a} T_{a, j}$. Será imposto que os $v_{j}$, valores esperado de vácuo de cada campo de ligação, sejam ordenados $v_{1}>v_{2}>\cdots>v_{N}$, com a seguinte parametrização em termos de $\mathrm{q}$, sendo $0<q<1$ e $\mathrm{v}$ o corte UV da teoria.

$$
v_{j}=v q^{j} .
$$

Portanto, os VEV decrescem de acordo que o valor de j aumenta de 0 a N. Logo, os VEVs serão localizados no Quiver de maneira decrescente para cada sítio a medida que j aumenta. Finalmente, a matriz de massa não diagonal é dada abaixo a partir dos termos de massa (2.1.0.13) com os VEVs ordenados (2.1.0.14).

$$
\Delta \mathcal{L}_{\text {mass } a}=\frac{1}{2} A_{\mu}^{a \dagger} M_{g}^{2} A^{\mu a},
$$

onde os autovetores dos bósons de gauge são definidos por $A_{\mu}^{a} \equiv\left\{A_{\mu, 0}^{a}, A_{\mu, 1}^{a} \ldots A_{\mu, N}^{a}\right\}$. Deste modo, a matriz de massa deles será dada por:

$$
M_{g}^{2}=\frac{g^{2} v^{2}}{2}\left(\begin{array}{ccccccc}
q^{2} & -q^{2} & 0 & 0 & \cdots & 0 & 0 \\
-q^{2} & q^{2}+q^{4} & -q^{4} & 0 & \cdots & 0 & 0 \\
0 & -q^{4} & q^{4}+q^{6} & q^{6} & \cdots & 0 & 0 \\
\vdots & \vdots & \vdots & \vdots & \ddots & \vdots & \vdots \\
0 & 0 & 0 & 0 & \cdots & q^{2(N-1)}+q^{2 N} & -q^{2 N} \\
0 & 0 & 0 & 0 & \cdots & -q^{2 N} & q^{2 N}
\end{array}\right)
$$


Deve-se realizar uma transformação unitária para os autoestados de massa $A_{\mu n}^{\prime}$, com o objetivo de diagonalizar a matriz (2.1.0.16).

$$
A_{\mu, j}=\sum_{n=0}^{N} f_{j, n} A_{\mu n}^{\prime}
$$

Para encontrar as equações de movimento, é necessário utilizar a Equação de EulerLagrange :

$$
\frac{\partial \mathcal{L}}{\partial A_{v, j}}-\partial_{\mu}\left(\frac{\partial \mathcal{L}}{\partial\left(\partial_{\mu} A_{v, j}\right)}\right)=0
$$

Supondo por simplicidade apenas o caso abeliano, as equações de Euler-Lagrange são aplicadas à Lagrangiana que descreve a teoria U(1):

$$
\mathcal{L}=\sum_{n=0}^{N}-\frac{1}{4} F_{\mu v, j} F_{j}^{\mu v}+\frac{g^{2}}{2} \operatorname{Tr}\left[v_{j}^{2}\left(A_{\mu}^{j}-A_{\mu}^{j-1}\right)^{2}\right],
$$

o que dá origem à equação de movimento:

$$
\partial_{\mu}\left(\partial^{\mu} A^{v}-\partial^{v} A^{\mu}\right)+g^{2}\left[v_{j}^{2}\left(A^{\mu, j}-A^{\mu, j-1}\right)-v_{j+1}^{2}\left(A^{\mu, j+1}-A^{\mu, j}\right)\right]=0 .
$$

No entanto, as equações de movimento para os autoestados de massa $A_{n}^{\prime \mu}$ são obtidas ao substituir os estados rotacionados de acorto com a equação (2.1.0.17) na equação acima.

$$
\left(\partial^{2} \delta_{\mu v}-\partial_{\mu} \partial^{v}\right) f_{j, n} A_{n}^{\prime \mu}+g^{2} v^{2} q^{2 j}\left(-q^{2} f_{j-1, n}+\left(1+q^{2}\right) f_{j, n}-q^{2} f_{j+1, n}\right) A_{n}^{\prime \mu}=0 .
$$

Por outro lado, $A_{v}^{\prime \mu}$ são autoestados de massa e devem satisfazer:

$$
\left(\partial^{2} \delta_{\mu v}-\partial_{\mu} \partial^{v}\right) A_{n}^{\prime \mu}+m_{n}^{2} A_{v}^{\prime \mu}=0 .
$$

Ao tomar o gradiente da equação acima (2.1.0.22) observa-se que

$$
\partial_{\nu} A_{\mu}^{\prime v}=0
$$

Observa-se que os autoestados de massa devem respeitar a equação (2.1.0.22) que é equivalente à equação de Proca:

$$
\partial^{2} A_{v}^{\prime \mu}+m_{n}^{2} A_{n}^{\prime v}=0
$$

Deve-se substituir então este resultado (2.1.0.23) na equação de movimento (2.1.0.21) obtendo o seguinte resultado:

$$
-m_{n}^{2} f_{j, n}+g^{2} v^{2} q^{2 j}\left(-q^{2} f_{j-1, n}+\left(1+q^{2}\right) f_{j, n}-q^{2} f_{j+1, n}\right) A_{n}^{\prime \mu}=0 .
$$


Ao definir $x_{n}$ tal que

$$
x_{n} \equiv \frac{m_{n}}{g v}
$$

pode-se escrever a equação de movimento como uma equação de diferenças, assim como feito em [28].

$$
\left[q+q^{-1}-x_{n}^{2} q^{-(2 j+1)}\right] f_{j, n}-q^{-1} f_{j-1, n}-q f_{j+1, n}=0 .
$$

A fim de encontrar soluções para as equações de movimento (2.1.0.25), são definidas as funções em termos do sítio j:

$$
\begin{aligned}
t[j] & =x_{n} q^{-j}, \\
F(t[j]) & =q^{j} f_{j, n} .
\end{aligned}
$$

Resultando na equação de q-diferenças:

$$
\left[q+q^{-1}-q^{-1} t^{2}\right] F(t)-F\left(q^{-1} t\right)-F(q t)=0 .
$$

É possível resolver analiticamente tais equações de q-diferenças (2.1.0.28). Isso é verdade devido ao fato delas serem um caso específico da Equação de Hahn-Exton para $v=1$, o que é uma analogia discreta às equações de Bessel que tem por soluções funções q-Bessel e q-Neumann. Estas tem propriedades parecidas com as funções de Bessel e Neumann, sendo que as funções contínuas podem ser recuperadas no limite $q \rightarrow-1$, correspondente ao limite $N \rightarrow \infty$. Essas soluções são descritas na referência [28]. Funções de q-Bessel são descritas da seguinte maneira:

$$
J_{v}(t ; q)=t^{v} \frac{\left(q^{v+1} ; q\right)_{\infty}}{(q ; q)_{\infty}} \sum_{i=0}^{\infty} \frac{(-1) q^{i(i+1) / 2}}{\left(q^{v+1} ; q\right)_{i}(q ; q)_{i}} t^{2 i}
$$

Onde $(p ; q)_{k}$ são fatoriais q-deslocados, definidos por:

$$
(p ; q)_{k}=\left\{\begin{array}{ll}
1 & \text { se } k=0 \\
\prod_{n=0}^{k-1}\left(1-p q^{n}\right) & \text { se } k \geq 1
\end{array}\right\},
$$

sendo os parâmetros p e q números complexos, $\mathrm{k}$ inteiro positivo $k=0,1,2, \ldots$, e o limite $(p, q)_{\infty} \equiv \lim _{k \rightarrow \infty}(p, q)_{k}$.

Já as Funções de q-Neumann são descritas por:

$$
Y_{v}(t ; q)=\frac{\Gamma_{q}(v) \Gamma_{q}(1-v)}{\pi} q^{-v^{2} / 2}\left[\cos (\pi v) q^{v / 2} J_{v}(t ; q)-J_{-v}\left(t q^{-v / 2} ; q\right)\right],
$$

onde a q-Extensão da função de Euler $\Gamma(v)$ é dada por:

$$
\Gamma_{q}(v)=\frac{(q ; q)_{\infty}}{\left(q^{v} ; q\right)_{\infty}}(1-q) 1-v,
$$

sendo que as funções $\Gamma_{q}(v)$ tem por limite $\Gamma(v)$ função Gama de Euler: 


$$
\lim _{q \rightarrow 1^{-}} \Gamma_{q}(v)=\Gamma(v) .
$$

A equação de diferenças (2.1.0.28) corresponde a $v=1$ para a equação de HahnExton. Logo, sua solução pode ser escrita como:

$$
F(t[j])=A J_{1}\left(t ; q^{2}\right)+B Y_{1}\left(t ; q^{2}\right),
$$

com A e B constantes. Para $f_{j, n}$ com condições de contorno tipo Neumann na função de onda dos bósons (2.1.0.25):

$$
\begin{array}{r}
f_{0, n}=f_{-1, n}, \\
f_{N+1, n}=f_{N, n} .
\end{array}
$$

Obtém-se :

$$
f_{j, n}=N_{n} q^{-} j\left[J_{1}\left(x_{n} q^{-j} ; q^{2}\right)+\beta\left(x_{n} ; q^{2}\right) Y_{1}\left(x_{n} q^{-j} ; q^{2}\right)\right]=0,
$$

sendo $N_{n}$ a normalização das funções $f_{j, n}$, e a parametrização $\beta\left(x_{n} ; q^{2}\right)$ dada por:

$$
\beta\left(x_{n} ; q^{2}\right)=-\frac{J_{0}\left(x_{n} ; q^{2}\right)}{Y_{0}\left(x_{n} ; q^{2}\right)} .
$$

Logo, o espectro de massas é descrito por:

$$
J_{0}\left(x_{n} ; q^{2}\right) Y_{0}\left(q^{-N+1} x_{n} ; q^{2}\right)-Y_{0}\left(x_{n} ; q^{2}\right) J_{0}\left(q^{-(N+1)} ; q^{2}\right)=0 .
$$

Isto prova analiticamente que para um grande número de sitios obtemos um modelo indistinguivel às teorias em $A d S_{5}$. Entretanto, na prática será usado $\mathrm{N}$ pequenos para os quais é possível resolver as equações numericamente.

\subsubsection{Função de onda dos bósons no modo zero}

Com a finalidade de calcular os acoplamentos entre a torre de bósons de gauge nas Teorias Quiver com férmions do MP é preciso demonstrar uma maneira simples de descrever as funções de onda do modo zero dos bósons. Este cálculo será feito em detalhe no próximo capítulo e será necessário para caracterizar a fenomenologia das Teorias Quiver no LHC.

Deve-se ressaltar que a função de onda dos bósons, a qual depende dos coeficientes $f_{j, n}$, satisfaz a equação de movimento descrita na equação (2.1.0.25). Nota-se que o determinante da matriz de massas para os bósons de gauge é nulo (2.1.0.16), o que também pode ser visto como a existencia de uma simetria $S U(m)$ não quebrada, isto explica um autovalor de massa nulo $m_{0}=0$. Logo, para $n=0$, a equação de movimento (2.1.0.25) é dada :

$$
\left[q+q^{-1}\right] f_{j, 0}-q^{-1} f_{j-1,0}-q f_{j+1,0}=0 .
$$


Para que seja possível determinar a forma do modo zero é preciso impor condições de contorno tipo Neumann (2.1.0.33) na função de onda dos bósons (2.1.0.25). Obtémse a condição de contorno:

$$
f_{j+1,0}=f_{j, 0} \equiv f_{0}
$$

com a condição de normalização:

$$
\sum_{j=1}^{N} f_{j, n}^{2}=1
$$

Então, para $n=0$

$$
\sum_{j=1}^{N} f_{0}^{2}=1 \Longrightarrow f_{0}=\frac{1}{\sqrt{N+1}} .
$$

O modo zero dos bósons de gauge na Teoria Quiver é constante. Isto é análogo ao caso contínuo, onde as funções de onda do modo zero dos bósons de gauge na dimensão extra é plana. Deste modo foi descrito o modo zero para o auto estado de massa dos bósons de gauge na Teoria Quiver. Este cálculo será usado no próximo capítulo para encontrar os acoplamentos necessários para definir a fenomenologia das teorias Quiver, desenvolvida no capítulo seguinte.

\subsection{Inclusão dos férmions na Teoria Quiver}

A contribuição fermiônica para a ação (2.1.0.1) é dada por:

$$
S_{f}=\int d^{4} x \sum_{j=0}^{N}\left\{\bar{\psi}_{L}^{j} i \not D_{j} \psi_{L}^{j}+\bar{\psi}_{R}^{j} i \not D_{j} \psi_{R}^{j}-\left(\mu_{j} \bar{\psi}_{L}^{j} \psi_{R}^{j}+\lambda_{j} \bar{\psi}_{R}^{j-1} \Phi_{j} \psi_{L}^{j}+\text { h.c. }\right)\right\}
$$

Os férmions vetoriais $\psi_{L / R, j}$ na Teoria Quiver se transformam na representação fundamental em relação aos grupos $G_{j}$.Ao incluí-los na ação (2.1.0.1), são adicionados nos diagramas moose linhas verticais em cada sítio representando os férmions de mão esquerda e direita para cada modo $\psi_{L, j}$ e $\bar{\psi}_{R, j}$, para $j=0,1, \ldots, N$. Deste modo, linhas verticais saindo em cada sítio correspondem à representação fundamental, enquanto as que entram correspondem à representação antifundamental em relação ao grupo de simetria do seu respectivo sítio.

Além disso, as linhas na diagonal que liga dois sítios representam o acoplamento do Yukawa dos férmions $\bar{\psi}_{R}^{j-1} \psi_{L}^{j}$ com os campos de ligação $\Phi_{j}$. É importante ressaltar que uma das linhas de férmions nas extremidades não se encontra no esquema, pois na ausência de $\bar{\psi}_{R, n}$, o modo zero será de mão esquerda, enquanto a falta de $\psi_{L, 0}$ implica no modo zero de mão direita, o que será explicado em mais detalhe no próximo capítulo. 


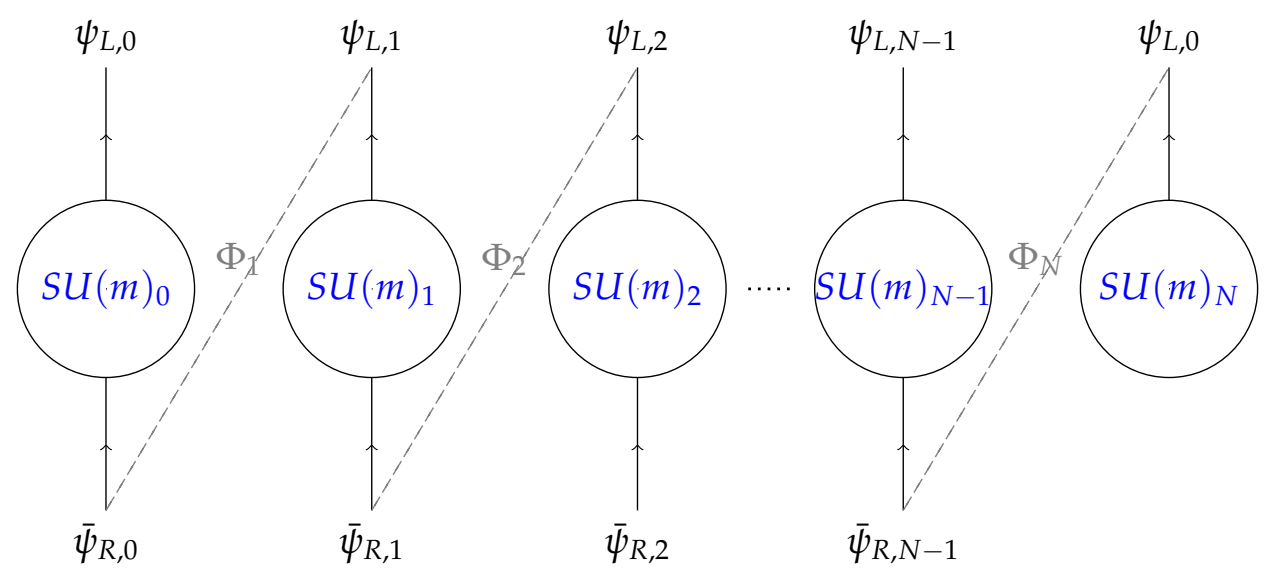

Figura 2.2: Diagrama de quiver com férmions representando $\mathrm{N}$ grupos de gauge conectados em uma cadeia linear.

Os termos de massa para os férmions são dados tanto pelo termo vetorial quanto pelo de Yukawa na ação (2.2.0.5). As massas dos férmions $\mu_{j}$ preservam a simetria de gauge, dado que são massas vetoriais. Por outro lado, observa-se que os termos de Yukawa são dados pela interação entre férmions de mão direita do sítio j e férmions de mão esquerda do sítio j+1. Então, os campos de ligação são representados ao conectar férmions de mão direita no sítio j com os de mão esquerda no sítio seguite. Isso é representado pelas linhas diagonais no diagrama quiver acima, as quais são chamadas de direção de salto. Além disso, esses termos são invariantes pois seu acoplamento depende do sítio e se trasforma sob:

$$
\Phi_{j} \rightarrow g_{j-1} \Phi_{j} g_{j}
$$

Novamente, o campo de ligação adquire um VEV:

$$
\Phi_{j} \rightarrow \frac{v_{j}}{\sqrt{2}^{\prime}}
$$

onde é utilizado o gauge unitário a fim de obter a matriz de massa dos férmions, sendo seus termos na Lagrangiana:

$$
\mathcal{L}_{m_{f}}=\sum_{j=0}^{N}\left(\mu_{j} \bar{\psi}_{j}^{L} \psi_{j}^{R}+\text { h.c. }\right)+\sum_{j=1}^{N} \lambda v_{j}\left(\bar{\psi}_{j-1}^{R} \psi_{j}^{L}+\text { h.c. }\right) \equiv \bar{\Psi}_{L} M_{f} \Psi_{R}+\text { h.c., }
$$

onde os férmions são representados por vetores da seguinte maneira:

$$
\Psi_{R, L}=\left(\begin{array}{llll}
\psi_{L, 0} & \psi_{L, 1} & \ldots & \psi_{L, N}
\end{array}\right)^{T} .
$$

Logo, a matriz de massa dos férmions não diagonal é dada por: 


$$
M M^{T}=\left(\begin{array}{cccccc}
\mu_{0}^{2} & \frac{\lambda}{\sqrt{2}} \mu_{0} v_{1} & 0 & \cdots & 0 & 0 \\
\frac{\lambda}{\sqrt{2}} \mu_{0} v_{1} & \left(\frac{\lambda}{\sqrt{2}}\right)^{2} v_{1}^{2}+\mu_{1}^{2} & \frac{\lambda}{\sqrt{2}} \mu_{1} v_{2} & \cdots & 0 & 0 \\
0 & \frac{\lambda}{\sqrt{2}} \mu_{1} v_{2} & \left(\frac{\lambda}{\sqrt{2}}\right)^{2} v_{2}^{2}+\mu_{2}^{2} & \cdots & 0 & 0 \\
\vdots & \vdots & \vdots & \ddots & \vdots & \vdots \\
0 & 0 & 0 & \cdots & \left(\frac{\lambda}{\sqrt{2}}\right)^{2} v_{N-1}^{2}+\mu_{N-1}^{2} & \frac{\lambda}{\sqrt{2}} \mu_{N-1} v_{N} \\
0 & 0 & 0 & \cdots & \frac{\lambda}{\sqrt{2}} \mu_{N-1} v_{N} & \mu_{N}^{2}
\end{array}\right) .
$$

Novamente, é preciso realizar a diagonalização sob a transformação unitária:

$$
\psi_{L, R}^{j}=\sum_{n=0}^{N} h_{L, R}^{j, n} \psi_{L, R}^{\prime n} .
$$

As equações de movimento são obtidas a partir das equações de Euler-Lagrange (2.1.0.18) para $\bar{\psi}_{j}^{R, L}$ através da ação com férmions (2.2.0.5):

$$
\begin{aligned}
\operatorname{para} \bar{\psi}_{R, j}: & i \not \partial \psi_{R, j}+\mu_{j} \psi_{L, j}+\lambda v_{j+1} \psi_{L, j+1}=0, \\
\text { para } \bar{\psi}_{L, j}: & i \not \partial \psi_{L, j}+\mu_{j} \psi_{R, j}+\lambda v_{j} \psi_{L, j-1}=0 .
\end{aligned}
$$

Ambos $\psi_{j}^{R, L}$ devem satisfazer a equação de Dirac, o que permite reescrever o termo cinético acima:

$$
i \not \partial \psi_{j}^{R, L}-m_{n} \psi_{j}^{R, L}=0 .
$$

A equação de movimento (2.2.0.10) na base diagonal, obtida pela expansão dos campos (2.2.0.9), resulta em:

$$
\begin{array}{r}
m_{n} h_{j, n}^{R}+\mu_{j} h_{j, n}^{L}+\lambda v_{j+1} h_{j+1, n}^{L}=0, \\
m_{n} h_{j, n}^{L}+\mu_{j} h_{j, n}^{R}+\lambda v_{j} h_{j-1, n}^{R}=0 .
\end{array}
$$

Ao desacoplar as equações de movimento (2.2.0.13) obtém-se:

$$
\begin{array}{r}
\left(\mu_{j}^{2}+\lambda^{2} v_{j}^{2}-m_{n}^{2}\right) h_{j, n}^{L}+\lambda \mu_{j} v_{j+1} h_{j+1, n}^{L}+\lambda \mu_{j-1} v_{j} h_{j-1, n}^{L}=0, \\
\left(\mu_{j}^{2}+\lambda^{2} v_{j+1}^{2}-m_{n}^{2}\right) h_{j, n}^{R}+\lambda \mu_{j+1} v_{j+1} h_{j+1, n}^{R}+\lambda \mu_{j} v_{j} h_{j-1, n}^{R}=0 .
\end{array}
$$

Estas equações de movimento desacopladas serão utilizadas na seção 2.3.3 para encontrar uma solução analítica para as funções de onda dos férmions no Quiver $h_{j, n}^{L, R}$, análoga às soluções constatadas para os bósons. Além disso, as equações de movimento serão importantes para descrever a localização dos férmions no Quiver, cálculo demonstrado no próximo capítulo. 


\subsection{Limite do Contínuo}

Teorias Quiver, também chamadas de Teorias desconstruídas podem ser derivadas de uma teoria de dimensões extras compactas, apesar de serem puramente quadridimensionais. Estes modelos foram inicialmente propostos desta maneira por [29] e [30], e estão descritos em detalhe em [31] ou [32]. O processo de desconstrução dimensional se dá ao discretizar a dimensão extra. Assim é possível traçar uma correspondência entre os modelos. No limite do contínuo, onde $N \rightarrow \infty$, as Teorias Quiver são equivalentes à teorias com um cenário em $A d S_{5}$. Nesse caso, as duas teorias terão caracterísiticas similares. Por isso, nesse trabalho é escolhido analisar a fenomenologia em poucos sítios, com o propósito de obter resultados novos para a fenomenologia na escala $\mathrm{TeV}$, distintos de modelos em $A d S_{5}$.

\subsubsection{Randall-Sundrum}

São supostos modelos com uma dimensão extra compacta, os quais possuem métrica anti de Sitter (AdS) em 5 dimensões. Essas teorias são bem descritas na literatura, por exemplo em [33]. A métrica é descrita por:

$$
d s^{2}=e^{-2 k|y|} \eta_{\mu \nu} d x^{\mu} d x^{\nu}-d y^{2},
$$

onde k é a curvatura na dimensão extra e $\mu=0,1,2,3$. Além disso, por hipótese, utiliza-se a métrica de Minkowski 4D $\eta_{\mu v}=\operatorname{diag}(1,-1,-1,-1)$. Será utilizada a notação em que letras gregas correspondem a parâmetros com 4 dimensões e letras maiúsculas latinas para 5 dimensões. Deste modo, a métrica também pode ser escrita como:

$$
d s^{2}=g_{M N} d x^{M} d x^{N}
$$

com

$$
g_{M N}=\left(\begin{array}{cc}
g_{\mu \nu} & 0 \\
0 & -1
\end{array}\right)
$$

Neste caso,

$$
g_{\mu \nu}=e^{-2 k|y|} \eta_{\mu \nu}
$$

É importante ressaltar que a dimensão extra compacta está localizada em um orbifold, com simetria $S^{1} / Z_{2}$. É preciso realizar o mecanismo de orbifolding a fim de eliminar os modos zero dos campos fermiônicos, já que estes não são observados no Modelo Padrão. Sua simetria é obtida ao relacionar os pontos de um hemisfério um círculo com o outro, ou seja, impõe-se que $y \leftrightarrow-y$, gerando uma reta com condição de periodicidade

$$
y+2 \pi R N=y
$$

Onde R é o raio da dimensão extra. Logo, a simetria $S^{1}$ é reduzida a $Z_{2}$, portanto é gerada a simetria compacta desejada como mostra a figura abaixo. Ou seja, $S^{1} / Z_{2}$ 


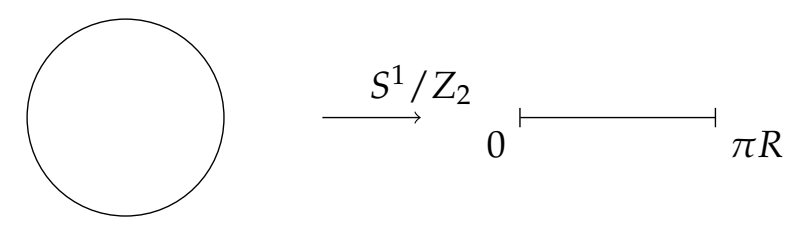

Figura 2.3: Ilustração do mecanismo de orbifolding.

é um círculo onde os pontos nas antípodas são identificados, o que resulta em um segmento de comprimento $L=\pi R$.

A partir da métrica $A d S_{5}$ (2.3.1.1), observa-se que a energia em $y=0$ é suprimida exponencialmente para $y \neq 0$. Logo, são identificados os pontos no orbifold $y=0 \mathrm{e}$ $y=\pi R$ como branas com energias diferentes, respectivamente serão os limites UV e IV da teoria. Enquanto as demais localizações dentro da dimensão extra estarão na chamada bulk. Desta maneira, uma partícula poderá estar localizada em uma das branas, ou se propagar dentro da bulk dependendo das propriedades que ela deverá apresentar.

A ação com uma dimensão extra pode ser escrita como:

$$
S_{5}=\int d^{4} x \int_{0}^{\pi R} d y \sqrt{g}\left(-\frac{1}{2 g_{5}^{2}} F_{M N} F^{M N}\right)+\ldots,
$$

onde os termos a não mencionados acima incluem férmions propagando na bulk em $A d S_{5}$ e $\sqrt{g}$ representa o determinante da métrica. Os tensores de intensidade do campo são descritos por:

$$
F_{M N} F^{M N}=F_{\mu v} F^{\mu v}+F_{5 \mu} F^{5 \mu}+F_{\mu 5} F^{\mu 5},
$$

com os campos 5 dimensionais:

$$
F_{5} \mu=\partial_{5} A_{\mu}-\partial_{\mu} A_{\mu}
$$

para o caso da teoria abeliana. Finalmente, a ação cinco dimensional para os bósons de gauge é descrita por:

$$
S_{5}^{g}=\int d^{4} x \int_{0}^{\pi R} d y \frac{1}{2 g_{5}^{2}}\left[-F_{\mu v} F^{\mu \nu}+e^{-2 k y}\left(\partial_{5} A_{\mu}^{a}-\partial_{\mu} A_{5}^{a}\right)^{2}\right]
$$

\subsubsection{Desconstrução dimensional}

Teorias Quiver são consideradas primas de teorias com uma dimensão extra compacta. No limite do contínuo, existe uma equivalência entre teorias desconstruídas e modelos em $A d S_{5}$. Esta correspondência pode ser obtida a partir do processo de 


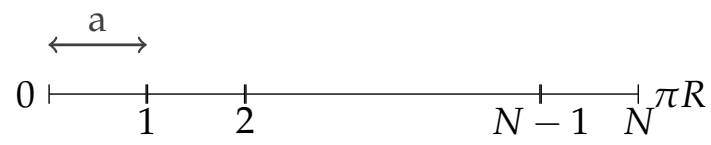

Figura 2.4: Ilustração da discretização da dimensão extra em N sítios de tamanho a.

desconstrução, onde a dimensão extra é discretizada de modo que se cria um dicionário entre componentes 5 dimensionais com parâmetros utilizados nos modelos no Quiver. O que foi descrito em [31] , [28] e [32] .

A ação cinco dimensional dada na equação (2.3.1.5) será discretizada em $\mathrm{N}$ intervalos correspondentes aos sítios no Quiver. Deste modo, o orbifold será separado por sítios de tamanho $a$, com as condições de contorno semelhantes ao caso em $A d S_{5}$. Analogamente, são identificados os sítios 0 como brana UV e N como brana IV.

Analiticamente, a discretização da dimensão extra corresponde à transformação da integral em dy pela somatória nos sítios, onde o tamanho infinitesimal dy corresponde à distância entre sítios $a$.

$$
\int_{0}^{\pi R} d y \rightarrow \sum_{j=0}^{N} a
$$

As derivadas correspondem à diferença infinitesimal entre os campos de gauge de cada sítio:

$$
\partial_{5} A_{\mu} \rightarrow \frac{A_{\mu}^{j}-A_{\mu}^{j-1}}{a},
$$

com

$$
A_{\mu}^{j} \equiv A_{\mu}\left(y_{j}\right),
$$

onde

$$
y_{j}=a \times j .
$$

A ação discretizada terá a forma:

$$
S_{d e c}=\int d^{4} x \sum_{j=0}^{N}\left(-\frac{a}{2 g_{5}^{2}} F_{\mu \nu}^{(j)} F^{\mu v(j)}\right)+\sum_{j=1}^{N} \frac{a}{2 g_{5}^{2}} e^{-2 k a j}\left(\frac{A_{\mu}^{j}-A_{\mu}^{j-1}}{a}-\partial_{\mu} A_{5}^{j}\right)^{2} .
$$

Com o objetivo de reaver a correspondência da teoria 5 dimensional com a quadridimensional, deve-se comparar a ação acima com a ação das Teorias Quiver (2.1.0.1). Sendo que foi utilizado o termo cinético da Lagrangiana após a quebra espontânea de simetria (2.1.0.7). O que resulta na seguinte ação para as Teorias Quiver:

$$
S_{\text {Quiver }}=\int d^{4} x\left\{-\sum_{j=0}^{N} \frac{1}{2 g^{2}} \operatorname{Tr}\left[F_{\mu \nu}^{(j)} F^{\mu v(j)}\right]+\sum_{j=1}^{N} \frac{1}{2}\left[\partial_{\mu} \pi_{j}+v_{j} g\left(A_{\mu, j-1}-A_{\mu, j}\right)\right]^{2}\right\} .
$$


A comparação entre as duas ações desconstruída, uma a partir de um cenário $A d S_{5}$ (2.3.2.3), a outra no Quiver (2.3.2.4), leva à conclusão de que o campo $A_{5}$ corresponde ao bóson de Nambu-Goldstone $\pi_{j}$ na Teoria Quiver. Também é possível construir um dicionário entre as duas teorias ao equiparar ambas as ações. Logo, a relação entre os dois termos cinéticos leva à correspondência:

$$
\frac{a}{g_{5}^{2}} \rightarrow \frac{1}{g^{2}}
$$

Já o termo de massa dos bosons de gauge, com $A_{\mu, j} \rightarrow \frac{1}{g} A_{\mu, j}$, dão lugar à relação

$$
\frac{1}{g_{5}^{2}} \times \frac{1}{a^{2}} e^{-2 k a j} \rightarrow v_{j}^{2}
$$

Substituindo os VEV $v_{j}$ pela sua distribuição ordenada ao longo dos sítios dada pela equação (2.1.0.14), as relações (2.3.2.5) e (2.3.2.6) dão origem ao dicionário entre as duas teorias, definido por:

$$
\begin{aligned}
\frac{g_{5}^{2}}{a} & \leftrightarrow g^{2} ; \\
v^{2} \leftrightarrow \frac{1}{\sqrt{a} g_{5}} & =\frac{1}{a g} ; \\
e^{-k a} & \leftrightarrow q .
\end{aligned}
$$

Uma vez construído o dicionário entre o caso quadridimensional e os modelos com uma dimensão extra é possível analisar o limiar entre essas teorias e determinar onde elas se tornam equivalentes. As teorias desconstruidas no limite de $a \rightarrow 0$ e $N \rightarrow \infty$ recuperam o caso contínuo. Para determinar esta fronteira, observa-se que a localização $q$ depende do tamanho $N a=\pi R \equiv L$ da dimensão extra, além da curvatura $k$, como visto na equação 2.3.2.9. O maior valor dessa localização multiplicada pelo cutoff da teoria, o qual pode ir até a massa de Planck, deve ser da ordem da escala eletrofraca para que a teoria seja observada e gere a devida hierarquia.

$$
e^{-k L} M_{\text {Planck }} \sim v_{E W} \sim 1 \mathrm{TeV},
$$

onde $M_{\text {Planck }} \sim 10^{19} \mathrm{GeV}$, então $e^{-k L} \sim 10^{-16} \mathrm{GeV}$ devido à hierarquia gerada entre a escala eletrofraca e o cutoff tipicamente na escala de Planck.

$$
e^{-k L} \simeq \frac{v_{E W}}{M_{\text {Planck }}}=10^{-16} .
$$

Logo, ao comparar a correspondência dada por (2.3.2.9) com a relação acima chegase à conclusão de que o parâmetro $q$ depende do número de sítios no Quiver da seguinte forma:

$$
q=10^{-16 / N}
$$




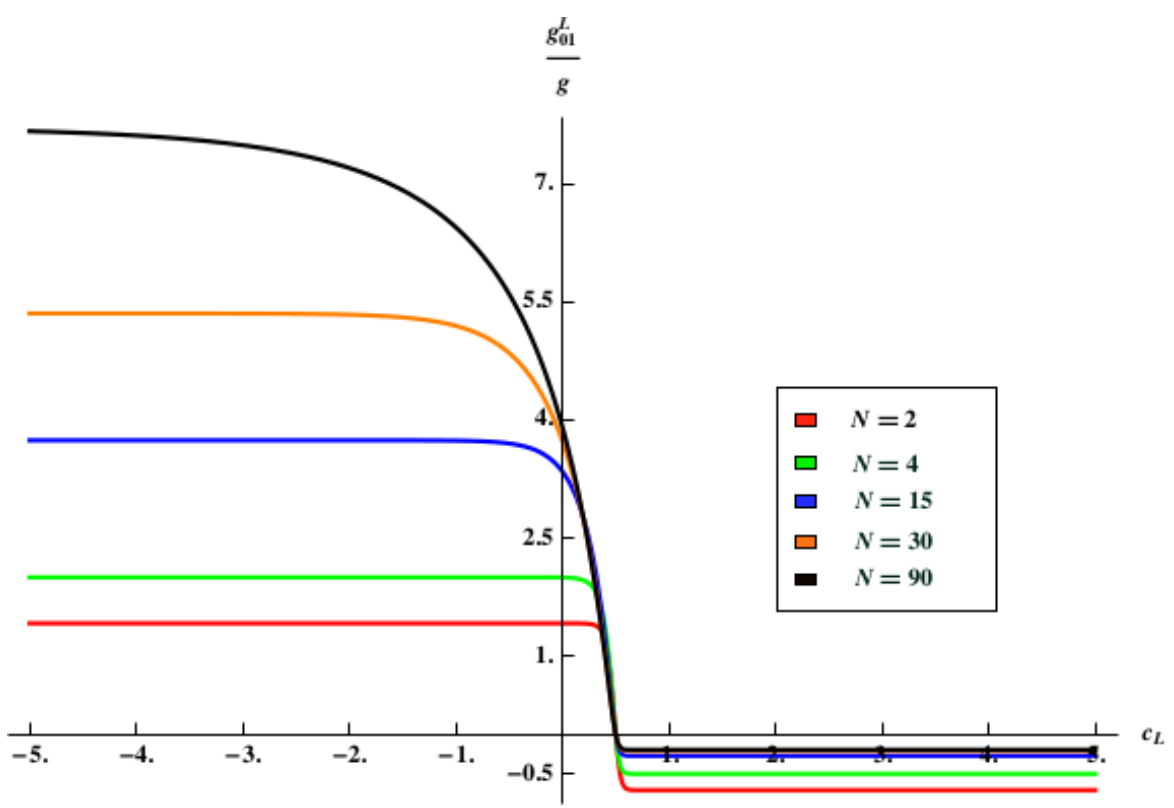

Figura 2.5: Acoplamento de mão esquerda do modo zero do férmion com o primeiro modo excitado do bóson de gauge, normalizado pelo acoplamento do modelo padrão $\frac{g_{01}^{L}}{g}$ para diferentes valores de N. Gráfico retirado da referência [25]

Por outro lado, se a curvatura k for comparada ao cutoff, então o tamanho dos sítios deve estar por baixo dessa escala de energia para que a teoria desconstruída seja caracteristicamente discreta. Pode se então dizer que o limite em que o contínuo é recuperado será nos casos em que:

$$
\begin{aligned}
& k \gg \frac{1}{a} \text { discreto; } \\
& k \ll \frac{1}{a} \text { contínuo. }
\end{aligned}
$$

Portanto, pode se chegar facilmente à conclusão de que $N>36$ para encontrar o limite em que a teoria deixa de ser discreta. Já que, tipicamente em um cenário de Randall-Sundrum $k L \simeq 36$, como $q \simeq e^{-k L}=e^{-k a N}$, é fácil ver que no limiar das duas teorias $k a \simeq 1$ e $N \simeq 36$. Para $N$ grande, ou seja $N \gg 36$ a teoria tem as mesmas características que RS. No entanto, numericamente, para $N=90$ a curvatura na figura 2.5 satura em $\sqrt{2 k \pi R}$ e é indistinguível da curva obtida para RS. A figura foi retirada da referência [25], sendo que o acoplamento do primeiro estado excitado do bóson de gauge com quarks do MP em função da localização c será calculado na seção 3.1.1. Além disso, o limite do contínuo também pode ser estudado na referência [34].

Por essas razões, a fenomenologia neste trabalho será analisada para Teorias Quiver 
com poucos sítios, já que para $\mathrm{N}$ grande, nesse caso $N>36$, a física que esse modelo implica será semelhante à dos modelos em Randall-Sundrum, os quais já foram muito bem analisados por outros trabalhos. Discussões sobre o limite do contínuo podem ser encontradas em referências como [28] e [35]. Isto posto, nesse trabalho será estudada a fenomenologia em poucos sítios, distante do contínuo, pois ela apresentará resultados distintos dos já vistos na literatura. No capítulo 4 será analisada a fenomenologia para 5, 10 e 16 sítios.

\subsubsection{Localização das funções de onda dos férmions}

A partir das equações de movimento dos férmions no Quiver (2.2.0.13), ao desacoplálas na equação (2.2.0.15), observa-se que as funções de onda serão descritas pelos parâmetros de massa $\mu_{j}$ e o acoplamento $\lambda$. Entretanto, é possível obter uma relação entre $\mu_{j}$ e $\lambda$ com a localização da função de onda dos férmions em cada sítio, analogamente ao feito em Teorias com Dimensões extras compactas. Ou seja, este resultado é obtido ao comparar a teoria no limite do contínuo com a ação 5-dimensional fermionica $S_{f}^{5}$, como foi feito no trabalho [31].

$$
S_{5}^{f}=\int d^{4} \int_{0}^{\pi R} d y e^{k y} i \bar{\Psi}_{L, R}^{\prime} \gamma^{\mu} \partial_{\mu} \Psi^{\prime}-\left(\Psi_{L}^{\prime} \partial_{5} \Psi_{R}^{\prime}-L \leftrightarrow R\right)+m_{\Psi}\left(\bar{\Psi}_{L}^{\prime} \Psi_{R}^{\prime}+\text { h.c. }\right) .
$$

Onde as funções de onda são definidas como $\Psi_{L, R}^{\prime}=e^{-2 k y} \Psi_{L, R}$ e a massa se relaciona com a curvatura $\mathrm{k}$ da seguinte maneira: $m_{\Psi} \equiv c k$, sendo c um parâmetro de ordem $o(1)$ que determinará a localização do modo zero do férmion na dimensão extra. Desta forma, é possível realizar a discretização, analogamente ao caso bosonico, inclusive com as derivadas cinco dimensionais indo para um limite de diferenças dos campos dividido pelo tamanho dos intervalos a.

$S_{5}^{f}=\int d^{4} \sum_{j=0}^{N} a\left[e^{k a j} i \bar{\psi}_{L, R} \gamma^{\mu} \partial_{\mu} \psi-\frac{1}{2 a}\left(\bar{\psi}_{R, j} \psi_{L, j+1}-\bar{\psi}_{L} \psi R, j-1+\right.\right.$ h.c. $)+c k\left(\bar{\psi}_{L} \psi_{R}+\right.$ h.c. $\left.)\right]$.

Assim como em teorias na rede, teorias com uma dimensão compacta discretizada apresentam o problema de duplicação dos férmions em teorias de gauge na rede. $\mathrm{Ou}$ seja, a Lagrangiana descrita em (2.3.3.2) gera dois modos zeros para os férmions. Entretanto, é possível resolver essa questão ao adicionar à ação o termo de Wilson. Ele resolve o problema para teorias discretas, dependendo da escolha de um parâmetro livre, e vai a zero no limite do contínuo. Este cálculo foi realizado para teorias desconstruídas primeiramente em [31].

Define-se um parâmetro c para férmions na Teoria Quiver. Esse parâmetro no limite do contínuo será o mesmo que descreve a localização na dimensão extra.

$$
\mu_{j} \leftrightarrow-g v_{j} q^{c+\frac{1}{2}}
$$


As equações de movimento descritas em (2.2.0.15) serão escritas em função das localizações c, com $x_{n}=\frac{m_{n}}{g v}$ e os valores esperados de vácuo $v_{j}$ ordenados (2.1.0.14). O que resulta nas equações de diferenças:

$$
\begin{array}{r}
{\left[q^{-\left(c+\frac{1}{2}\right)}+q^{\left(c+\frac{1}{2}\right)}-q^{-\left(c+\frac{1}{2}\right)}\left(x_{n} q^{-j+\frac{1}{2}}\right)^{2}\right] h_{j, n}^{L}-q h_{j+1, n}^{L}-q^{-1} h_{j-1, n}^{L}=0 ;} \\
{\left[q^{-\left(c-\frac{1}{2}\right)}+q^{\left(c-\frac{1}{2}\right)}-q^{-\left(c-\frac{1}{2}\right)}\left(x_{n} q^{-\left(j+\frac{1}{2}\right)}\right)^{2}\right] h_{j, n}^{R}-q h_{j+1, n}^{R}-q^{-1} h_{j-1, n}^{R}=0 .}
\end{array}
$$

Essas equações são equações de movimento nas Teorias Quiver para férmions. Assim como no caso bosonico, existe resoluções analíticas para essas equações. Elas são descritas por funções de q-Bessel e q-Neumann. Novamente é possível recuperar as soluções no limite do contínuo, em que as soluções vão para funções de Bessel e Neuman, as mesmas obtidas por modelos em $A d S_{5}$.

Em Teorias $A d S_{5}$ as partículas analisadas são localizadas na Bulk, ou seja, dentro do orbifold onde está a dimensão extra. Já para teorias desconstruídas, a localização toma a forma do valor do $h_{j, n}^{L, R}$. O parâmetro c poderá ser o análogo às localizações no diagrama de quiver. Logo, com o objetivo de calcular os acoplamentos na Teoria Quiver, determina-se a localização c para cada modo zero fermiônico ao relacioná-lo com suas massas. Essa relação decorre do fato das massas serem determinadas pelos acoplamentos com o Higgs, o qual estará localizado essencialmente no IV. 


\section{Acoplamento dos estados excitados}

Esse capítulo tem por objetivo demonstrar cálculos feitos nas Teorias Quiver, necessários para demonstrar a física que determinará a fenomenologia analisada no capítulo 4. Primeiramente, serão determinados os acoplamentos do modo zero fermiônicos, correspondentes à fermions do MP, com estados excitados do bóson de gauge. Esses acoplamentos caracterizarão a física das teorias desconstruídas para um número de sítios $\mathrm{N}$ pequeno.

Por outro lado,Teorias Quiver satisfazem os vínculos de violação de sabor para $M_{G}>$ $3 \mathrm{TeV}$. Além do mais, não há necessidade de estender o setor de gauge a fim de evitar violação da simetria custodial já que os vínculos de precisão eletrofraca são satisfeitos nessa escala de massa, como demonstrado na referência [25]

Finalmente, será descrito o Higgs como um pseudo bóson de Nambu Goldstone, de modo que este será localizado majoritariamente no sítio IV. Cálculos envolvendo o bóson de Higgs não afetarão o estudo da fenomenologia nesse trabalho. No entanto é necessário demonstrar como Teorias Quiver solucionam o problema de hierarquia ao descrever o bóson de Higgs com um pseudo bóson de Nambu Goldstone.

\subsection{Acoplamentos para o modo zero dos férmions}

Essa seção tem por objetivo mostrar o cálculo feito para procurar os acoplamentos que caracterizarão a fenomenologia em Teorias Quiver. Será possível definir nesse capítulo os acoplamentos dos quarks, representados por modos zero dos férmions na Teoria Quiver, com o primeiro modo excitado dos bósons de gauge. Os acoplamentos dependerão da localização no diagrama de quiver, a qual pode ser definida uma vez que a matriz de massa e mistura CKM dos quarks são bem conhecidas. 


\subsubsection{Acoplamentos em função da localização}

O objetivo dessa seção é descrever os acoplamentos g̃ vindo da interação entre o modo zero de dois férmions e o primeiro modo de excitado do bóson de gauge. Serão descritos os acoplamentos g̃ em função da localização no Quiver de cada férmion. A Lagrangiana desa interação é dada por:

$$
\mathcal{L}=\sum_{n=0}^{N} \tilde{\mathrm{g}} \bar{\psi}_{L, n} \gamma^{\mu} A_{\mu, n} \psi_{L, n}
$$

reenforçando que g̃ é o acoplamento entre o primeiro modo excitado do bóson de gauge com o modo zero fermiônico. Deve-se descrever a teoria na base diagonal dos férmions e autoestados de massa dos bósons. Ao expandir a Lagrangiana respectivamente de acordo com as equações (2.1.0.17) e (2.2.0.9), obtém-se:

$$
\mathcal{L}=\tilde{\mathrm{g}} \sum_{j, k, m=0}^{N} \sum_{n=0}^{N}\left[\left(h_{n, j}^{L}\right)^{*} f_{n, m} h_{n, k}^{L}\right] \bar{\psi}_{L, j}^{\prime} \gamma^{\mu} A_{\mu, m}^{\prime} \psi_{L, k}^{\prime}
$$

onde $A_{\mu, m}^{\prime}$ são autoestados de massa dos bósons de gauge e $\psi_{L / R, j}^{\prime}$ são os férmions já rotacionados para a base diagonal. É possível identificar o acoplamento do modo j e $\mathrm{k}$ dos férmions com o modo $\mathrm{m}$ do bóson de gauge:

$$
g_{j, k, m}=\tilde{\mathrm{g}} \sum_{n=0}^{N}\left[\left(h_{n, j}^{L}\right)^{*} f_{n, m} h_{n, k}^{L}\right]
$$

Logo, para o modo zero dos férmions $j=k=0$, o acoplamento é dado por:

$$
g_{0,0, m}=\tilde{\mathrm{g}} \sum_{n=0}^{N}\left|h_{n, 0}^{L}\right|^{2} f_{n, m}
$$

Desta maneira pode-se definir o acoplamento g, que corresponde ao acoplamento no MP entre férmions e respectivos bósons, relacionando-o com g̃:

$$
g \equiv g_{0,0,0}=\tilde{\mathrm{g}} \sum_{n=0}^{N}\left[\left|h_{n, 0}^{L}\right|^{2} f_{n, 0}\right]
$$

Dado que a função de onda bosônica no modo zero $f_{n, 0}$ é constante (2.1.1.4), descrevese o acoplamento (3.1.1.5), com as funções dos férmions $h_{n, 0}^{L, R}$ normalizadas, segundo a equação:

$$
\sum_{n=0}^{N}\left|h_{n, 0}^{L}\right|^{2}=1 .
$$

Obtém-se a relação entre o acoplamento g do MP e o acoplamento novo g̃ para os demais estados excitados caracterizados pela Teoria Quiver: 


$$
g=\tilde{g} \frac{1}{\sqrt{N+1}} .
$$

O acoplamento dos modo zero dos férmions com um modo $\mathrm{m}$ do campo de gauge dado pela equação (3.1.1.4) pode ser descrito em termos do acoplamento no MP g, e do número de sítios $\mathrm{N}$, ao substituir g de acordo com a equação(3.1.1.7):

$$
g_{0,0, m}=g \sqrt{N+1} \sum_{n=0}^{N}\left|h_{n, 0}^{L}\right|^{2} f_{n, m} .
$$

Por outro lado, a partir da equação de movimento para as funções de onda dos férmions (2.2.0.13), obtemos para o modo zero, com $m_{0}=0$ :

$$
\begin{aligned}
\mu_{j} h_{j, 0}^{L}+\lambda v_{j+1} h_{j+1,0}^{L} & =0, \\
\mu_{j} h_{j, 0}^{R}+\lambda v_{j} h_{j-1,0}^{R} & =0 .
\end{aligned}
$$

Ao escrever a relação entre os modos zero, tanto de mão esquerda quanto direita, pode-se definir os parâmetros de localização $c_{R}$ e $c_{L}$ da seguinte maneira:

$$
\begin{gathered}
\frac{h_{j, 0}^{L}}{h_{j+1,0}^{L}}=-\frac{\lambda v_{j+1}}{\mu_{j}} \equiv q^{-\left(c_{L}-1 / 2\right)}, \\
\frac{h_{j, 0}^{R}}{h_{j-1,0}^{R}}=-\frac{\lambda v_{j}}{\mu_{j}} \equiv q^{-\left(c_{R}+1 / 2\right)} .
\end{gathered}
$$

A partir das equações acima (3.1.1.11) e (3.1.1.12) para os primeiros dois sítios, com $N=0$ e $N=1$, obtemos a relação:

$$
h_{1,0}^{R}=Z^{R, L} h_{0,0}^{R},
$$

onde $Z^{R, L}$ são definidos através das localizações no quiver $c_{R, L}$.

$$
\begin{array}{r}
Z^{R} \equiv q^{-\left(c_{R}+\frac{1}{2}\right)}, \\
Z^{L} \equiv q^{c_{L}-\frac{1}{2}} .
\end{array}
$$

Iterando (3.1.1.13) $N-1$ vezes, chega-se no resultado que relaciona o enésimo estado excitado do férmion com seu modo zero por meio da sua localização no Quiver,

$$
h_{n, 0}^{R, L}=\left(Z^{R, L}\right)^{n} h_{0,0}^{R, L} \text {. }
$$

Ao considerar a normalização das funções de onda fermiônicas dada pela equação (3.1.1.6), é possível compará-la à equação acima (3.1.1.16) e obter: 


$$
\left|h_{0,0}^{L, R}\right|^{2} \sum_{k=0}^{N}\left(Z^{R, L}\right)^{2 n}=1 .
$$

Nota-se que a soma acima na equação (3.1.1.17) não passa de uma soma de progressão aritmética em $\left(Z^{R, L}\right)^{2}$, de modo que é possível escrever as funções $h^{L, R} \mathrm{em}$ termos dos parâmetros de localização através de $Z^{L, R}$ da seguinte maneira:

$$
\left|h_{0,0}^{L, R}\right|^{2}=\left(\frac{1-\left(Z^{R, L}\right)^{2}}{1-\left(Z^{R, L}\right)^{N+1}}\right)^{\frac{1}{2}} .
$$

Em seguida, esse resultado descrito na equação (3.1.1.18) é substituido no acoplamento dos modos zero dos férmions de mão esquerda $\frac{g^{L}}{g}$ em (3.1.1.8). Esse processo é feito com a finalidade de obter o acoplamento de mão esquerda ou direita $\frac{g_{L, R}}{g}$, dependente das localizações $c_{L, R}$, do número de sítios $N$ e das autofunções de massa dos bósons de gauge $f_{n, m}$.

$$
g_{0,0, m}^{L, R}=g \sqrt{N+1} \sum_{n=0}^{N}\left(Z^{R}\right)^{2 n}\left(\frac{1-\left(Z^{R, L}\right)^{2}}{1-\left(Z^{R, L}\right)^{N+1}}\right)^{\frac{1}{2}} f_{n, m} .
$$

Finalmente, a interação entre dois férmions do MP e o primeiro estado excitado do bóson de gauge é dado pelo acoplamento abaixo:

$$
g_{0,0,1}^{L, R}=g \sqrt{N+1} \sum_{n=0}^{N}\left(Z^{R}\right)^{2 n}\left(\frac{1-\left(Z^{R, L}\right)^{N+1}}{1-\left(Z^{R, L}\right)^{2}}\right)^{-\frac{1}{2}} f_{n, 1} .
$$

Dado um número de sítios, é possível observar seu comportamento em função da sua localização na figura 2.5. Assim como já discutido na seção 2.3.2, para $N>36$ as curvas obtidas correspondem ao caso do limite contínuo. No caso de dimensões extras, assim como no quiver com muitos sítios existe o problema da violação de sabor em correntes neutras, discutidos mais adiante na seção 3.3. Entretanto, também será mostrado adiante nesse capítulo que as curvas com poucos sítios formam um platô, permitindo que os acoplamentos dos quarks com localizações diferentes sejam mais universais.

\subsubsection{Acoplamento de Yukawa}

É necessário descrever o acoplamento de Yukawa dos férmions em função da localização $c_{R, L}$ para que seja possível determinar os acoplamentos do modo excitado dos bósons de gauge às partículas do MP. Os valores de $c_{L, R}$ são obtidos ao comparar cálculos feitos a partir das massas e matriz CKM, pressupondo acoplamentos de Yukawa de ordem 1. Os acoplamentos de Yukawa físicos dos modos zero são hierárquicos devido à localização no quiver.

Por outro lado, para de resolver o problema de hierarquia, o acoplamento de $\mathrm{Yu}$ kawa com o Higgs deve ser totalmente localizado no sítio IV o que será discutido 
em maior detalhe na próxima seção 3.2. Para efeitos práticos do cálculo das massas e matriz CKM, pode-se fazer a aproximação em que os férmions só se acoplam com o Higgs no sítio N. Logo, a Lagrangiana que descreve a interação entre o campo escalar $\phi$ e férmions de modo zero é dada apenas pelo modo $\mathrm{N}$ dos férmions:

$$
\mathcal{L}_{Y}=\bar{\psi}_{L, N} Y H \psi_{R, N}+\text { h.c., }
$$

onde $Y_{i j}$ primordial é de ordem 1 . Utilizando os férmions de modo zero na base diagonal (2.2.0.9), em função das localizações $Z_{R} Z_{L}$, (3.1.1.16)

$$
\mathcal{L}_{Y}=\left(Z_{L}\right)^{N} h_{0,0}^{L}\left(Z_{R}\right)^{N} h_{0,0}^{R} \bar{\psi}_{L, 0} Y H \psi_{R, 0}+\text { h.c. }
$$

Substituindo no resultado acima a expansão em (3.1.1.18) , obtém-se a interação de Yukawa da forma:

$$
\begin{aligned}
\mathcal{L}_{Y}=\left(Z_{L}\right)^{N}\left(Z_{R}\right)^{N}\left(\frac{1-\left(Z^{L}\right)^{2}}{1-\left(Z^{L}\right)^{N+1}}\right)^{\frac{1}{2}} & \\
& \left(\frac{1-\left(Z^{R}\right)^{2}}{1-\left(Z^{R}\right)^{N+1}}\right)^{\frac{1}{2}} \bar{\psi}_{L, 0} Y H \psi_{R, 0}+\text { h.c.. }
\end{aligned}
$$

Ao comparar a interção de Yukawa acima com o acoplamento inicial dos modos zero em (3.1.2.1), define-se a matriz de Yukawa que corresponde ao MP por:

$$
Y_{0} \equiv\left(Z_{L}\right)^{N}\left(Z_{R}\right)^{N}\left(\frac{1-\left(Z^{L}\right)^{2}}{1-\left(Z^{L}\right)^{N+1}}\right)^{\frac{1}{2}}\left(\frac{1-\left(Z^{R}\right)^{2}}{1-\left(Z^{R}\right)^{N+1}}\right)^{\frac{1}{2}} Y .
$$

Observa-se que o acoplamento efetivo de Yukawa dos modos zero depende da localização dos férmions $c_{L, R}$ através das funções $Z^{L, R}$. A localização dos modos zero fermiônicos determina a hierarquia das massas dos férmions do MP, com parâmetros $c_{L, R}$ e $Y$ de ordem 1.

\subsection{Localização do Higgs}

Nessa seção será descrita a localização do Bóson de Higgs utilizando Teorias Quiver. É importante ressaltar que esse cálculo não será usado para descrever a fenomenologia, mas é preciso mencioná-lo pois este resolve o problema de hierarquia, além de justificar o uso de um Higgs localizado no sítio N na equação (3.1.2.1). Para solucionar essas questões, o bóson de Higgs deve estar majoritariamente localizado no IV, análogo ao caso das teorias de Randall-Sundrum. Nas Teorias Quiver, sua localização é gerada dinâmicamente ao quebrar as simetrias dos grupos de gauge.

Os grupos de simetria de gauge no quiver serão escolhidos de modo que ao menos 4 bNG não são absorvidos, o que dá origem aos graus de liberdade identificados com os dubletos do Higgs após a quebra espontânea de simetria no Quiver. O Higgs é 


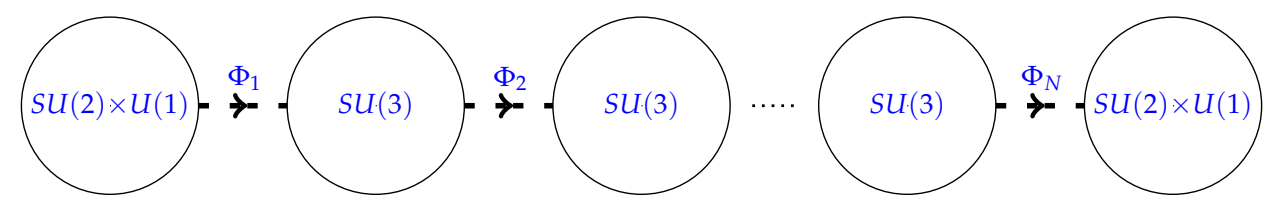

Figura 3.1: Diagrama de quiver em $\mathrm{N}$ sítios numa cadeia linear. Nota-se que nem todos os geradores da simetria global $S U(3)$ são gaugeados, já que os sítios das extremidades são substituídos por $S U(2) \times U(1)$.

descrito como um pseudo bóson de Nambu Goldstone, analogamente ao píon na QCD. No caso do píon, existe uma simetria global: a simetria quiral $S U(2)_{L} \times S U(2)_{R}$. A diferença entre a massa hadrônica $m_{\rho} \sim 770 \mathrm{MeV}$ e a massa do píon $m_{\pi} \sim 140 \mathrm{MeV}$ é explicada pela quebra espontânea de simetria, dada pela simetria axial vetorial, que é equivalente à de mão esquerda e direita fermiônica, com $S U(2)_{A} \times S U(2)_{V} \rightarrow$ $S U(2)_{V}$. Logo, $S U(2)_{A}$ é espontaneamente quebrada, resultando em $3 \mathrm{bNG}$, os quais corresponderão aos píons $\pi^{+}, \pi^{0}$ e $\pi^{-}$. A quebra espontânea de simetria é responsável por gerar a diferença $m_{\pi} \ll m_{\rho}$, no entanto ela implica em um píon com massa zero. A fim de obter píons massivos, é possível realizar uma quebra explícita de simetria, ela é pequena o suficiente para preservar $m_{\pi} \ll m_{\rho}$. A descrição do píon como um pseudo bNG pode ser estudada através da referência [5].

Ao descrever um modelo de teorias de gauge com a estrutura dos grupos de simetria do tipo

$$
G_{0} \times G_{1} \times G_{2} \ldots G_{j} \times G_{j+1} \ldots G_{N}
$$

eles devem conter uma simetria de gauge que abrange o grupo $S U(2) \times U(1)$ como subgrupo. A escolha mais simples seria escolher todos os grupos $G_{j}$ como $S U(3)$. Entretanto, neste caso todos os bósons de gauge serão absorvidos na quebra de simetria $S U(3)_{j-1} \times S U(3)_{j} \rightarrow S U(3)_{\text {diag }}$ pelos campos de ligação $\phi_{j}$. Concluí-se que os graus de liberdade que serão correspondidos ao dubleto de Higgs, por isso, é preciso reduzir a simetria de gauge em ao menos 4 geradores. Os grupos $G_{j}$ serão escolhidos de modo que o primeiro e último grupo sejam simplesmente $S U(2) \times U(1)$ e os demais grupos de gauge sejam $S U(3)$, conforme o diagrama de quiver abaixo. Desta maneira, os sítios 0 e $\mathrm{N}$ com a simetria $S U(2) \times U(1)$ não apresentam os bósons de gauge associados à simetria $S U(3) / S U(2) \times U(1)$, sobrando 4 geradores não quebrados associados aos graus de liberdade do Higgs.

A quebra de simetria $S U(3)_{j-1} \times S U(3)_{j} \rightarrow S U(3)_{\text {diag }}$ pelos campos de ligação $\phi_{j}$ gera um octeto de bósons de Nambu-Goldstone $\pi^{A} T^{A}$, sendo $T^{A}$ os geradores de $S U(3)$. Os oito geradores do grupo SU(3) são dados por $T^{a}=\frac{\lambda^{a}}{2}, \operatorname{com} \lambda^{a}$ matrizes de Gell-Mann, dados por: 


$$
\begin{aligned}
\lambda^{1}=\left(\begin{array}{lll}
0 & 1 & 0 \\
1 & 0 & 0 \\
0 & 0 & 0
\end{array}\right) \lambda^{2}=\left(\begin{array}{ccc}
0 & -i & 0 \\
i & 0 & 0 \\
0 & 0 & 0
\end{array}\right) & \lambda^{3}=\left(\begin{array}{ccc}
-1 & 0 & 0 \\
0 & 1 & 0 \\
0 & 0 & 0
\end{array}\right) \\
\lambda^{4}=\left(\begin{array}{lll}
0 & 0 & 1 \\
0 & 0 & 0 \\
1 & 0 & 0
\end{array}\right) \lambda^{5}=\left(\begin{array}{ccc}
0 & 0 & -i \\
0 & 0 & 0 \\
i & 0 & 0
\end{array}\right) & \\
\lambda^{6}=\left(\begin{array}{lll}
0 & 0 & 0 \\
0 & 0 & 1 \\
0 & 1 & 0
\end{array}\right) \lambda^{7}=\left(\begin{array}{ccc}
0 & 0 & 0 \\
0 & 0 & -i \\
0 & i & 0
\end{array}\right) & \lambda^{8}=\frac{1}{\sqrt{3}}\left(\begin{array}{ccc}
1 & 0 & 0 \\
0 & 1 & 0 \\
0 & 0 & -2
\end{array}\right) .
\end{aligned}
$$

Ao substituir os dois grupos das pontas por $S U(2) \times U(1)$, não existe um dado conjunto de geradores. Para diferenciar os geradores não quebrados de $S U$ (3) é preciso denotar os geradores dos grupos de $S U(2) \times U(1)$ como $Y^{A}=\left\{T^{1}, T^{2}, T^{3}, T^{8}\right\}$, enquanto os geradores de $S U(3) / S U(2) \times U(1)$ será descrito por $X^{A}=\left\{T^{4}, T^{5}, T^{6}, T^{7}\right\}$. Com essa nova escolha de grupos de gauge no quiver é possível calcular o termo cinético. Para isto, ele é expandido em termos do VEV e $\pi_{j}^{a}$ analogamente à (2.1.0.4):

$$
\begin{aligned}
\mathcal{L}_{\text {cin }}^{\pi}=D_{\mu} \phi_{j} D^{\mu} \phi_{j}=\frac{1}{2 v_{j}^{2}} \partial_{\mu} \pi_{j}^{A} \partial^{\mu} \pi_{j}^{A} & \\
& +\frac{1}{v_{j}} \partial_{\mu} \pi_{j}^{A}\left(g_{j-1} A_{\mu j-1}^{A}-g_{j} A_{\mu j}^{A}\right)+\text { h.c. } \\
& +\left[g_{j-1}^{2}\left(A_{\mu j-1}^{A}\right)^{2}+g_{j}^{2}\left(A_{\mu j}^{A}\right)^{2}\right]-\frac{g_{j-1} g_{j}}{2} A_{\mu j-1}^{A} A_{\mu j}^{A},
\end{aligned}
$$

onde deve-se separar o primeiro e o último termo correspondentes aos sítios nas pontas que não contém todos os geradores de $S U(3)$ :

$$
\begin{gathered}
\mathcal{L}_{c i n}^{\pi}=\frac{1}{2 v_{1}^{2}}\left[\partial_{\mu} \pi_{1}^{\alpha}-v_{1} \tilde{\mathrm{g}} A_{\mu 1}^{\alpha}\right]^{2}+\sum_{j=2}^{N-1} \frac{1}{2 v_{j}^{2}}\left[\partial_{\mu} \pi_{j}^{\alpha}-v_{j} \tilde{\mathrm{g}}\left(A_{\mu j}^{\alpha}-A_{\mu, j-1}^{\alpha}\right)\right]^{2} \\
+\frac{1}{2 v_{N}^{2}}\left[\partial_{\mu} \pi_{N}^{\alpha}-v_{N} \tilde{\mathrm{g}} A_{\mu N}^{\alpha}\right]^{2} .
\end{gathered}
$$

Será adicionado o termo de fixação de gauge:

$$
\mathcal{L}_{G}=-\sum_{j=1}^{N-1} \frac{1}{2 \xi}\left[\partial_{\mu} A_{j}^{\mu \alpha}-\xi \tilde{g}\left(v_{j} \pi_{j}^{\alpha}-v_{j+1} \pi_{j+1}^{\alpha}\right)\right]^{2} .
$$

Logo, obtém-se as matrizes de massa para os bósons de gauge a partir do termo de massa que pode ser adquirido na Lagrangiana em (3.2.0.9), com os valores esperados 
de vácuo ordenados descritos como em (2.1.0.14).

$$
M_{A}^{2}=\tilde{g}^{2} v^{2}\left(\begin{array}{ccccccc}
q^{2}+q^{4} & -q^{4} & 0 & 0 & \cdots & 0 & 0 \\
-q^{4} & q^{4}+q^{6} & -q^{6} & 0 & \cdots & 0 & 0 \\
0 & -q^{6} & q^{6}+q^{8} & -q^{8} & \cdots & 0 & 0 \\
\vdots & \vdots & \vdots & \vdots & \ddots & \vdots & \vdots \\
0 & 0 & 0 & 0 & \cdots & \left.q^{2(N-2)}+q^{2(N-1)}\right) & -q^{2 N} \\
0 & 0 & 0 & 0 & \cdots & -q^{2 N} & q^{2(N-1)}+q^{2 N}
\end{array}\right) .
$$

Para os bósons de Nambu Goldstone, descreve-se os termos de massa a partir do termo de fixação de gauge (3.2.0.10), o que resulta na matriz de massas:

$$
M_{\pi}^{2}=g^{2} v^{2} \xi\left(\begin{array}{ccccccc}
q^{2} & -q^{3} & 0 & 0 & \cdots & 0 & 0 \\
-q^{3} & 2 q^{4} & -q^{5} & 0 & \cdots & 0 & 0 \\
0 & -q^{5} & 2 q^{6} & -q^{7} & \cdots & 0 & 0 \\
\vdots & \vdots & \vdots & \vdots & \ddots & \vdots & \vdots \\
0 & 0 & 0 & 0 & \cdots & 2 q^{2(N-1)} & -q^{2 N-1} \\
0 & 0 & 0 & 0 & \cdots & -q^{2 N-1} & q^{2 N}
\end{array}\right)
$$

Diferente ao caso descrito no capítulo 2, descrito pela matriz de massas dos bósons de gauge em (2.1.0.16), a matriz $M_{\pi}^{2}$ tem determinante zero, e portanto existe um modo zero. Para que seja possível descrever a função de onda do Higgs a função de onda do modo zero será expandida na base dos autoestados:

$$
H^{\alpha}=h_{j} \pi^{\alpha} .
$$

As funções $h_{j}$ satisfazem a relação de recursão abaixo, vinda da equação (3.2.0.12)

$$
h_{j}=q h_{j+1} .
$$

Impondo a normalização

$$
\sum_{j=1}^{N}\left|h_{j}\right|^{2}=1,
$$

obtém-se a função de onda do Higgs:

$$
H^{\alpha}=\sum_{j=1}^{N} \frac{q^{N-j}}{\sqrt{\sum_{j=1}^{N} q^{2(j-1)}}} \pi_{j}^{\alpha} .
$$

Supondo um valor de $q$ pequeno, pois $q \simeq 10^{-16 / N}$, é possível observar que para poucos sítios o Higgs estará quase totalmente no sítio N. Enquanto no limite do contínuo, para grandes valores de $\mathrm{N}$, há uma solução semelhante à observada em teorias com uma dimensão extra compacta. A função de onda do Higgs nesse caso tem um valor 
muito maior perto da brana IV e é suprimida conforme se afasta dela, ou seja, para sítios com $j<N$.

Por fim, o bóson de Higgs foi descrito como um pseudo Bóson de Nambu Goldstone. Ele adquire um potencial devido ao fato das interações do MP quebrarem a simetria global que protegem $m_{H}$. Foi escolhida a simetria descrita nessa seção a fim de resolver o problema de hierarquia. A altas energias, o sistema recupera a simetria global, ou seja, a simetria exata $S U(3)$ impede divergências quadráticas. Enquanto a localização no sítio $\mathrm{N}$ implica em um Higgs leve, com acoplamento $\lambda_{t}$ pequeno. Logo, o cálculo da correção à massa do Higgs a um loop será limitado, como visto em (1.2.1.2) ao explicar o problema de hierarquia na seção 1.2.1. Entretanto, o corte da teoria não será tão alto como em $M_{\text {Planck, }}$ podendo gerar nova física na escala $\mathrm{TeV}$.

\subsection{Localização dos férmions no Quiver}

A fim de determinar os acoplamentos do modo zero dos férmions ao primeiro modo excitado do bóson de gauge é necessário designar as localizações $c_{L, R}$ destes. Essas terão como parâmetros físicos as massas e ângulos de mistura dos quarks, vindos da matriz CKM. Logo, é possível procurar a posição dos quarks do MP no diagrama quiver com poucos sítios.

Além disso, as soluções encontradas devem minimizar a violação de sabor e estar de acordo com os experimentos de precisão eletrofraca. Em teorias com uma dimensão extra compacta há o problema de grande violação de sabor. Nas teorias desconstruídas, para um alto número de sítios há a mesma questão, já que o modelo se aproxima do caso em $A d S_{5}$. Os vínculos de sabor para $A d S_{5}$ são de $M_{G}>10 \mathrm{TeV}$. Entretanto, para poucos sítios a fenomenologia é distinta e há pouca violação de sabor. As ressonâncias nas Teorias Quiver são fracamente acopladas, logo, os vínculos obtidos restringem a massa do estado excitado do bóson de gauge a $M_{G}>3 \mathrm{TeV}$.

\subsubsection{Correntes neutras com mudança de sabor}

No MP, os acoplamentos neutros são universais dentre os diversos sabores dos quarks. A Lagrangiana que descreve a interação entre o bóson $Z_{\mu}$ e quarks é descrita com um acoplamento $g_{Z}$ :

$$
\mathcal{L}_{Z_{\mu}}=g_{Z} Z_{\mu} \bar{Q}_{R}^{\dagger} \gamma^{\mu} Q_{R} .
$$

Onde $Q_{R, L}$ representam os spinores com as três famílias de quarks:

$$
Q_{R}=\left(\begin{array}{c}
u_{R} \\
c_{R} \\
t_{R}
\end{array}\right), Q_{L}=\left(\begin{array}{c}
u_{L} \\
c_{L} \\
t_{L}
\end{array}\right) .
$$

Entretanto, as interações de Yukawa geram termos de massa não diagonais entre as 


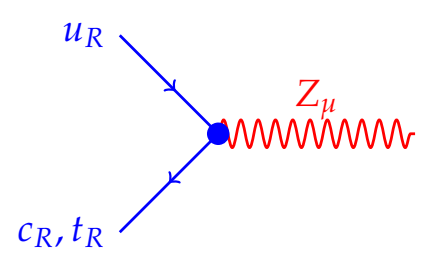

Figura 3.2: Exemplo de diagrama de Feynman com violação de sabor em correntes neutras.

famílias de quarks, dada por:

$$
\begin{array}{r}
\mathcal{L}_{Y}=\bar{Q}_{L} M_{Q} Q_{R} \\
M_{Q}=\lambda \frac{v}{\sqrt{2}}
\end{array}
$$

Logo, é necessário fazer uma rotação para a base diagonal de autoestados de massa:

$$
\begin{gathered}
\tilde{\mathrm{Q}}_{R}=U_{R} Q_{R} \\
\tilde{\mathrm{Q}}_{L}=U_{L} Q_{L}
\end{gathered}
$$

Onde a Lagrangiana pode ser descrita na base diagonal ao realizar a tranformação unitária $U_{L}, U_{R}$.

$$
\mathcal{L}_{Y} \rightarrow \bar{Q}_{L} U_{L}^{\dagger} M_{Q} U_{R} Q_{R}=Q_{L} M_{Q}^{\text {diagonal }} Q_{R}
$$

onde as massas dos quarks são descritas pela matriz diagonal $M_{Q}$.

$$
M_{Q}^{\text {diagonal }}=\left(\begin{array}{ccc}
m_{u} & 0 & 0 \\
0 & m_{c} & 0 \\
0 & 0 & m_{t}
\end{array}\right) .
$$

Portanto, a interação do $Z_{\mu}$ com os autoestados de massa não apresentam violação de sabor. Isso se dá ao fato de que a Lagrangiana $\mathcal{L}_{Z_{\mu}}$ é invariante sob a transformação de base dos spinores $Q_{L, R}$, já que as matrizes de rotação são unitárias, como pode se observar:

$$
\begin{aligned}
\mathcal{L}_{Z_{\mu}} & \rightarrow \mathcal{L}_{Z_{\mu}}^{\prime} \\
\mathcal{L}_{Z_{\mu}}^{\prime}=Z_{\mu} Q_{R} U_{R}^{\dagger} \gamma^{\mu} U_{R} Q_{R}=Z_{\mu} \bar{Q}_{R} Q_{R} & =\mathcal{L}_{Z_{\mu}}
\end{aligned}
$$

É certo que não existem a nível árvore diagramas que misturam quarks de sabores diferentes no MP. Logo, não serão encontrados diagramas como apresentado na figura 3.2. O que resulta na ausência de correntes neutras com violação de sabor. Fica claro que, no MP, não é possível ter mistura entre as famílias de quarks a nível árvore 
quando se trata de interações com os bósons de gauge $Z_{\mu}$, ou analogamente ao fóton $\gamma$ e glúon.

Entretanto, as Teorias Quiver permitem violação de sabor a nível árvore. Isso ocorre visto que os acoplamentos são distintos nas interações entre os bósons de gauge neutros e os quarks de gerações diferentes. Este efeito também é observado nas teorias com uma dimensão extra compacta. Assim sendo, é necessário observar que tal violação de sabor deve ser consistente com vínculos experimentais de precisão eletrofraca.

Para o primeiro estado excitado do bóson de gauge, a Lagrangiana que dá sua interação no quiver com os quarks do MP é descrita por:

$$
\mathcal{L}_{G^{(1)}}=G_{\mu}^{(1)}\left(g_{u} \bar{u}_{R} \gamma^{\mu} u_{R}+g_{c} \bar{c}_{R} \gamma^{\mu} c_{R}+g_{t} \bar{t}_{R} \gamma^{\mu} t_{R}\right) .
$$

Observa-se que, nesse caso, os acoplamentos dependerão da localização do seu respectivo quark no quiver. Esta diferença torna a Lagrangiana $\mathcal{L}_{G^{(1)}}$ em (3.3.1.11) sensível à transformação de base para os autoestados de massa descrita na equação (3.3.1.5). Ou seja, valores distintos dos acoplamentos para diferentes famílias deverão gerar violação de sabor. Estes acoplamentos tem um valor semelhante para os três sabores de quarks, com a intenção de diminuir a violação de sabor.

A figura 2.5 apresenta os acoplamentos do primeiro estado excitado do bóson de gauge com quarks, para diferentes valores do número de sítios N. A partir dessa figura, concluí-se que o resultado com mínima violação de sabor é obtido para poucos sítios. Isso é devido ao fato de que, para $\mathrm{N}$ grande, os acoplamentos se comportam da mesma maneira que as teorias com dimensões extras compactas: existe um platô no limite IV, entretanto a curva não é constante no limite UV. Por outro lado, para um número pequeno de sítios são formados dois platôs, tanto no IV, quanto no UV. Logo, é mais provável que as soluções para os acoplamentos tenham um valor aproximado entre si quanto menor o número de sítios.

Portanto, a fenomenologia de Teorias Quiver em poucos sítios deve ser distinta das Teorias com Dimensões Extras. Isso ocorre devido ao fato de haver menor violação de sabor para valores pequenos de $\mathrm{N}$. Os resultados obtidos, bem como os vínculos de sabor são descritos na próxima seção para $N=4$, o que equivale a 5 sítios.

\subsubsection{Violação de sabor no Quiver}

Foram obtidas soluções para as localizações $c_{R, L}$ dos quarks na Teoria Quiver em 5 sítios na referência [25]. Dado que o valor dos acoplamentos dos férmions do MP com o primeiro modo excitado dos bósons de gauge depende da sua posição no quiver. Isto posto, ao supor um valor para $c_{R, L}$ é possível calcular os acoplamentos de Yukawa segundo a equação (3.1.2.4), o que darão origem às massas e matriz de mistura para os quarks do MP, como foi visto nas equações (1.1.5.5), (1.1.5.4) e (1.1.5.9). Para isso, foi desenvolvido um algoritimo genético. Nele, uma população de soluções aleatórias são geradas dentre os valores possíveis das localizações $c_{L, R}$. $\mathrm{O}$ algoritimo 
então calcula para cada solução aleatória o quão próxima ela está dos dados experimentais de massa e da matriz CKM. Depois, a população é ordenada pelo fator de proximidade da solução adequada. Uma parte dela é descartada, a qual está mais longe da solução final, enquanto novas possibilidades randomicas são geradas. Finalmente, após um número suficiente de iterações, são encontradas soluções que representam as localizaçõs $c_{L, R}$ para para os modos zero dos férmions, ou seja, para cada família dos quarks do MP.

\section{Vínculos de sabor}

É necessário levar em consideração que a solução para as localizações $c_{R, L}$ dos quarks na Teoria Quiver em 5 sítios deve estar de acordo com os vínculos de violação de sabor. Eles são obtidos através de dados acumulados por experimentos de física de sabor, como Belle, Babar ou LHCb, os quais estão reunidos na referência [36]. Observase que para poucos sítios há pouca violação de sabor, compatível com os vínculos experimentais. No entanto, no limite do contínuo, a situação se assemelha à Teorias com uma dimensão extra compacta, onde há violação de sabor no setor down.

A solução encontrada para a localização de quarks a 5 sítios é descrita pelos gráficos apresentados nas figuras 3.3. Neste caso, observa-se que os acoplamentos de mão direita no setor down, descrito na figura 3.3b, estão localizados no mesmo platô UV para os três sabores de quark. Portanto, este setor está quase universalmente acoplado. Ou seja, para as três famílias de quark, o valor do acoplamento com o primeiro estado excitado será aproximadamente o mesmo, evitando a violação de sabor em correntes neutras.

Já para o acoplamento dos quarks de mão esquerda 3.3a e de mão direita no setor up 3.3c, há uma pequena violação de sabor, já que o acoplamento do top é maior que os demais, fato que resulta por sua massa ser muito maior que os quarks up e charm.

Experimentos de sabor geram vínculos para mistura de quarks no setor down, os quais estão discutidos em mais detalhe em [25] [26]. São considerados dados gerados pela medição da amplitude de oscilação $D^{0}-\bar{D}^{0}$ ou $K^{0}-\bar{K}^{0}$, calculada a um loop no MP, o qual é finito para 3 gerações de quarks. Pode-se relacionar os valores encontrados para o acoplamento de cada família de quarks com coeficientes de Wilson, que também dependerão da massa $M_{G}$. Ao comparar coeficientes de Wilson com os valores encontrados dos acoplamentos, chega-se à conclusão que a massa do modo excitado dos bósons deve ser limitada inferiormente, tal que:

$$
M_{G}>3 \mathrm{TeV} .
$$

\section{Vínculos de precisão eletrofraca}

É possível também analisar os parâmetros de precisão eletrofracos para testar a Teoria Quiver. Esse cálculo e discussão também podem ser achados em mais detalhe 


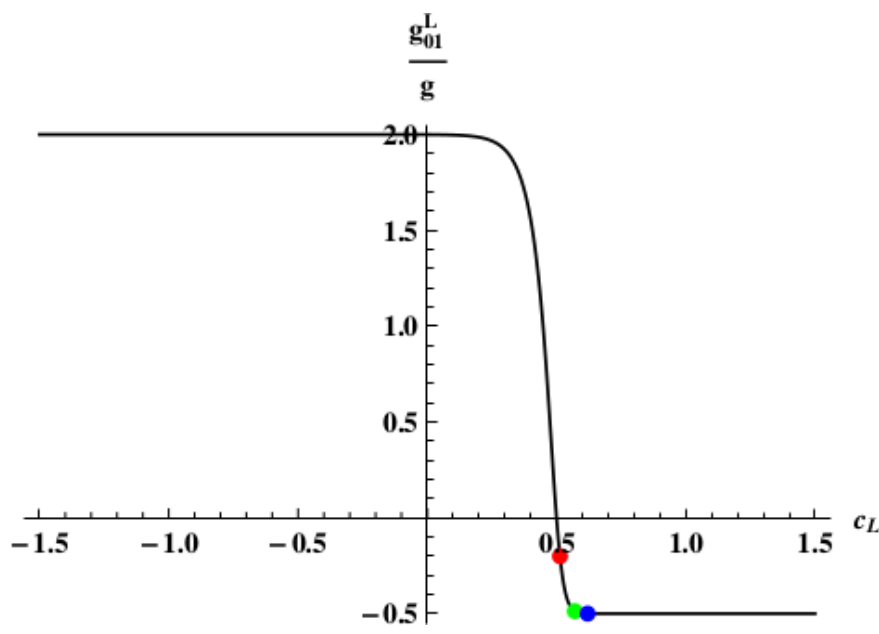

(a) Acoplamento de mão esquerda dos quarks com o primeiro modo excitado do bóson, normalizado pelo acoplamente do MP, em função da localização c.

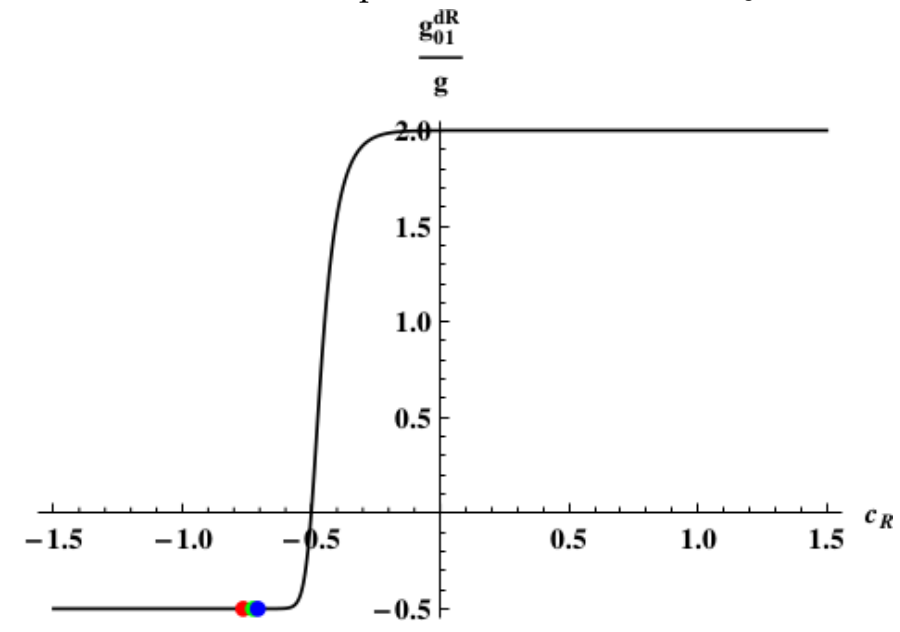

(b) Acoplamento de mão direita dos quarks no setor down com o primeiro modo excitado do bóson, normalizado pelo acoplamente do MP, em função da localização c.

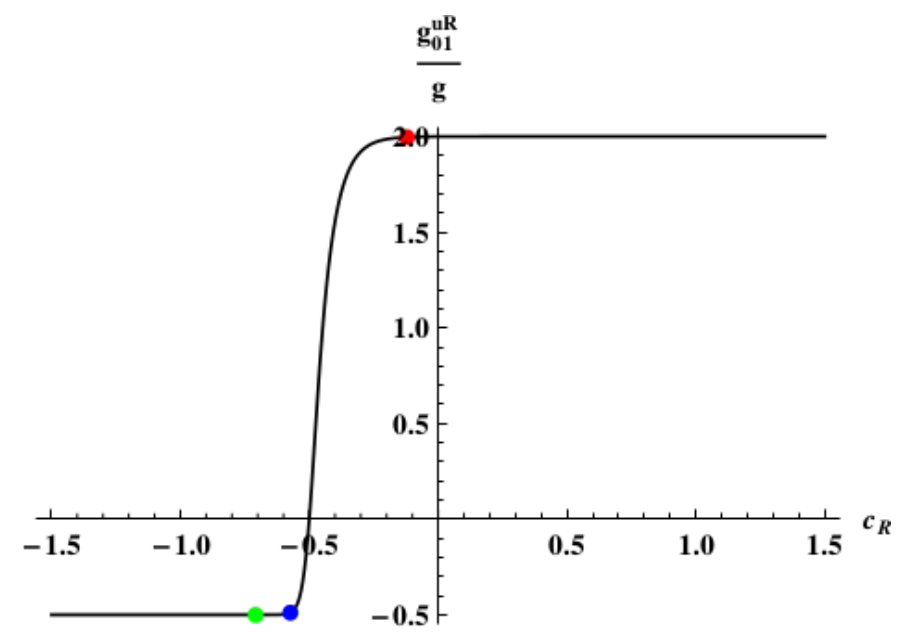

(c) Acoplamento de mão direita dos quarks no setor up com o primeiro modo excitado do bóson, normalizado pelo acoplamente do MP, em função da localização c.

Figura 3.3: Acoplamentos do modo zero dos férmion com o primeiro modo excitado do bóson, normalizado pelo acoplamente do MP, em função da localização c. As soluções para as três famílias dos quarks estão representadas pelos pontos coloridos. Gráficos retirados da referência [25] . 


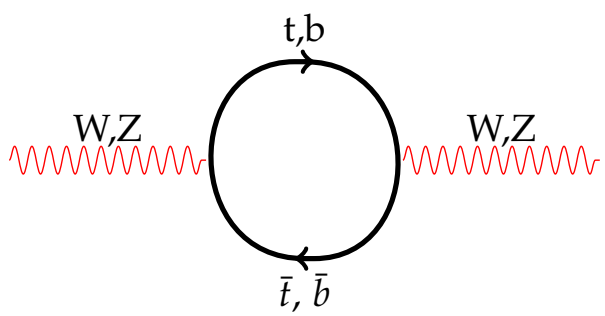

Figura 3.4: Os parâmetros de precisão eletrofraca S e T vêm da diferença de diagramas a um loop como o demonstrado na figura.

nas referências [25] e [26] . Serão consideradas as correções oblíquoas S, T e U, chamadas de parâmetros de Peskin-Tekeuchi. Sendo o parâmetro U normalmente desprezível, enquanto $\mathrm{S}$ e T são definidos por :

$$
\begin{array}{r}
S=16 \pi\left(\Pi_{33}^{\prime}(0)-\Pi_{3 Q}^{\prime}(0)\right) \\
T=\frac{4}{v^{2}}\left(\Pi_{11}(0)-\Pi_{33}(0)\right)
\end{array}
$$

Os parâmetros acima descrevem as diferenças das autoenergia ou função de polarização do vácuo dos bósons de gauge de interação fraca. Elas são calculadas através da soma de diagramas irredutiveis (1PI), resultando na amplitude $\Pi_{I J}^{\mu v}$, com I J correspondentes aos bósons de gauge eletrofracos $\gamma, W^{ \pm}$ou $Z^{0}$.

As correções às massas dos bósons $W^{ \pm}$e $Z^{0}$ darão origem respectivamente à $\Pi^{11,21} \mathrm{e}$ $\Pi^{33,3 Q}$, esse cálculo pode ser encontrada na referência [5]. Logo, o parâmetro T está diretamente correlacionado às correções entre os bósons de gauge em $S U(2)$ com transformações de Isospin, com $\mathrm{S}$ sendo sua derivada em relação ao momento. Além disso, o parâmetro $\mathrm{T}$ é definido a partir da diferença de diagramas com contribuições a um loop de pares de quarks top e bottom, o que representa justamente $\Pi_{W W} \sim \Pi_{11}$, o que está explicado detalhadamente na referência [37]. Existem contribuições aos parâmetros $\mathrm{S}$ e $\mathrm{T}$ vindo de misturas entre os bósons eletrofracos induzidos pelos estados excitados nas Teorias Quiver. Nas teorias quiver são encontrados diagramas a nível árvore contendo mistura dos estados excitados com os modos zeros dos bósons $W^{1,2,3}$, B. Logo, os cálculos considerando correções às partículas de interação do MP devem ser corrigidos também por estes diagramas. Apesar dessas contribuições serem pequenas ao parâmetro $S$, elas não podem ser desconsideradas no cálculo de T. A solução encontrada a poucos sítios dos parâmetros S e T pela referência [25]:

$$
\begin{aligned}
S & \simeq 0.17 \times\left(3 \mathrm{TeV} / M_{G}\right)^{2} \\
T & \simeq 0.16 \times\left(3 \mathrm{TeV} / M_{G}\right)^{2}
\end{aligned}
$$

Seus valores experimentais são bem conhecidos como $S_{\exp }=0.03 \pm 0.10$ e $T_{\exp }=$ $0.05 \pm 0.12$ [38]. Isto resulta em um vínculo para a massa do bóson de gauge modo um a $M_{G}>3 \mathrm{TeV}$. 
Os vínculos à massa do primeiro estado excitado do bóson de gauge $M_{G}$ vindo da física de sabor e os de medições de precisão eletrofraca, $\mathrm{S}$ e $\mathrm{T}$, são similares. Por essa razão, vínculos diretos do LHC serão decisivos para incrementar esse vínculo através da procura direta nos experimentos ATLAS e CMS. 


\section{4}

\section{Fenomenologia}

Neste capítulo é apresentado um estudo da fenomenogia em Teorias Quiver no LHC. O objetivo é testar esses modelos ao confrontá-los com os dados experimentais. Primeiramente, será calculada a seção de choque de produção do primeiro estado excitado do bóson de gauge no canal s. Serão analisados os diagramas de Feynman que representam a interação dos modos 1 de bósons de gauge gerados pela teoria desconstruída com partículas do modelo padrão. Logo, utiliza-se neste cálculo os acoplamentos apresentados no capítulo 3 para diferentes números de sítios, os quais caracterizarão a nova física que poderá ser gerada pela teoria.

São estudados tanto os estados excitados dos bósons eletrofracos quanto aqueles correspondentes ao grupo de cor. O setor eletrofraco deve conter no mínimo o grupo de quiver $G=\left[S U(2)_{L} \times U(1)_{Y}\right]^{N}$, ou algum outro grupo que obedeça a quebra espontânea da simetria eletrofraca no MP. Logo, é possível supor que o grupo de gauge $G=[S U(3)]^{N}$ também se propague no quiver, o qual é quebrado no grupo de gauge da QCD $G \rightarrow S U(3)_{C}$. Nesse caso, o modo zero do bóson de gauge corresponde ao Glúon do Modelo Padrão, e os modos seguintes são interpretados como uma torre de estados excitados, ou ressonâncias do octeto de cor de spin 1 massivos. Essa análise, assim como os vínculos de detecção direta estão na referência [1].

Finalmente, serão apresentados os vínculos obtidos com os dados acumulados pelo LHC. Foram analisados dados obtidos recentemente pelo LHC no CERN e publicados pelas colaborações CMS [2], [3] e ATLAS [4]. Nota-se que os vínculos de deteç̧ão direta estão de acordo com os retirados de experimentos de sabor e precisão eletrofraca. No entanto, deve-se aguardar novos dados com maior energia do centro de massas e luminosidade da próxima fase do LHC. Deste modo será possível obter vínculos mais restringentes ou encontrar nova física. 


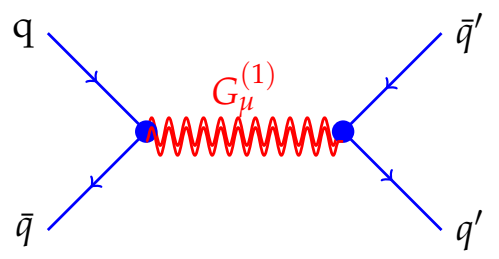

Figura 4.1: Diagrama de Feynman representando a produção e decaimento do primeiro estado excitado do bóson de gauge em quarks do MP no canal s.

\subsection{Seção de Choque partônica}

Serão considerados cálculos para a seção de choque partônica no canal s para o processo $q \bar{q} \rightarrow G^{(1)} \rightarrow q^{\prime} \bar{q}^{\prime}$. Ou seja, descreve-se a produção e decaimento do modo excitado dos bósons de gauge em quarks do modelo padrão. Os processos analisados neste trabalho serão apenas nos canais de decaimento em quark e antiquark, sendo que o setor leptônico deverá ser estudado no futuro, o que será discutido nas conclusões no capítulo 5 [19]. Para isso utilizamos os acoplamentos já calculados no capítulo 3 para a Teoria Quiver em um dado número de sítios. O resultado apresentado nessa seção é fundamental para o estudo da fenomenologia, já que ela se dá justamente ao comparar o cálculo da seção de choque multiplicada pela razão de ramificação ou branching ratio com o obtido experimentalmente. Este é representado pela largura vezes a seção de choque do processo analisado.

\subsubsection{Largura}

Será apresentado o cáculo da largura para o decaimento do estado excitado do bóson de gauge das Teorias Quiver. O cálculo aqui demonstrado será feito para o glúon $G^{(1)}$. No entanto, serão considerados também vínculos para os estados excitados dos bósons neutros $\gamma^{(1)}$ e $Z^{(1)}$. Eles devem decair em quarks do Modelo Padrão. Para o caso do glúon, isso ocorrerá através da interação $S U(3)$ mediada por um octeto de cor, a qual é descrita pela seguinte Lagrangiana:

$$
\mathcal{L}=g_{q} \bar{Q}_{q i} \gamma^{\mu} T_{i j}^{a} Q_{q j} G_{\mu}^{a}
$$

onde os acoplamentos $g_{q}$ dependem do sabor do quark, representados por $Q_{i}, i=$ 1,2,3. Além disso, $T^{a}$ são dados pelos geradores de $S U(3)$ do octeto de cor $G_{\mu}$. A largura é obtida ao somar sob todos os sabores de quarks a amplitude invariante $\mathscr{M}$ ao quadrado. Além disso, deve-se multiplicá-la pelo fluxo inicial e integrá-la no espaço de fases do decaimento do primeiro modo excitado do bóson de gauge em duas partículas. O resultado final é da forma:

$$
\Gamma=\sum_{q} \frac{\sqrt{\frac{M_{G}^{2}}{4}-m_{q}^{2}}}{32 \pi^{2} M_{G}^{2}}\left|\overline{\mathscr{M}}_{\Gamma}\right|^{2} 4 \pi,
$$


onde $\left|\overline{\mathscr{M}}_{\Gamma}\right|$ é o módulo da amlitude invariante, a qual será calculada adiante, além disso, $M_{G}$ e $m_{q}$ são as massas do bóson e quark respectivamente.

Para que a amplitude invariante $\mathscr{M}$ seja calculada, pode-se derivar as regras de Feynman para o vértice que dá a interação descrita em (4.1.1.1) entre o primeiro modo de um bóson de gauge $G_{\mu}^{(1)}$ e um par quarks e antiquark de quadrimomento k e k'. Logo, o vértice é representado pelo diagrama:

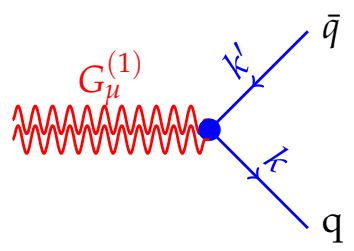

O diagrama acima corresponde ao vértice:

$$
-i T_{i j}^{a} \gamma^{\mu}\left(g_{V}^{q}-g_{A}^{q} \gamma^{5}\right)
$$

Os vérices são descritos a partir dos acoplamentos vetorial e axial de cada quark, relacionados aos acoplamentos de mão direita e esquerda pelas relações:

$$
\begin{aligned}
& g_{R}=g_{V}+g_{A}, \\
& g_{L}=g_{V}-g_{A} .
\end{aligned}
$$

A amplitude invariante será escrita como:

$$
i M_{\Gamma}=\bar{u}(k)_{i}(-i) T_{i j}^{a} \gamma^{\mu}\left(g_{V}^{q}-g_{A}^{q} \gamma^{5}\right) v\left(k^{\prime}\right)_{j} \epsilon_{\mu}^{a},
$$

onde $\mathrm{u}$ e $\mathrm{v}$ são os propagadores fermiônicos, dependentes dos momentos de cada quark, e $\epsilon_{\mu}^{a}$ corresponde à polarização do bóson de gauge. Ao tirar a média da amplitude, além de efetuar a soma sob spin, cor e sob a polarização $\epsilon_{\mu}$, obtém-se a amplitude quadrática:

$$
\begin{aligned}
\sum_{\text {spin,cor }}\left|M_{\Gamma}\right|^{2}= & \sum_{\lambda} \sum_{\text {spin,cor }}\left[\bar{u}(k)_{j}^{s^{\prime}} T_{j j^{\prime}}^{a}(-i) \gamma^{\mu}\left(g_{V}^{q}-g_{A}^{q} \gamma^{5}\right) v\left(k^{\prime}\right)_{j^{\prime}}^{s} \epsilon_{\mu}^{* a, \lambda}\right] \\
& \times\left[\epsilon_{v}^{b, \lambda} \bar{v}\left(k^{\prime}\right)_{i}^{s} T_{i i^{\prime}}^{b}(i) \gamma^{v}\left(g_{V}^{q}-g_{A}^{q} \gamma^{5}\right) u(k)_{i^{\prime}}^{s^{\prime}}\right] .
\end{aligned}
$$

A fim de calcular a amplitude acima em termos observáveis é preciso relevar uma série de relações entre os componentes analisados. Primeiro, é possível realizar a soma das polarizações e descrevê-la a partir do quadrimomento do bóson $P_{\mu}$ da seguinte maneira:

$$
\sum_{\lambda} \epsilon_{\mu}^{* a, \lambda} \epsilon_{v}^{b, \lambda}=\left(g_{\mu v}-\frac{P_{\mu} P_{v}}{M_{G}^{2}}\right) \delta_{a b} .
$$


Enquanto as relações de completeza das funções de onda fermiônicas serão somadas sob seus respectivos valores de spin.

$$
\begin{aligned}
\sum_{s^{\prime}} u(k)_{i^{\prime}}^{s^{\prime}} \bar{u}(k)_{j}^{s^{\prime}} & =\left(\not k+m_{q}\right) \delta_{i^{\prime} j}, \\
\sum_{s} v\left(k^{\prime}\right)_{j^{\prime}}^{s} \bar{v}\left(k^{\prime}\right)_{i}^{s} & =\left(\not k k^{\prime}-m_{q}\right) \delta_{i j^{\prime}} .
\end{aligned}
$$

Além disso toma-se a normalização dos traços dos geradores do grupo $S U(3)$, com $C(N)=\frac{1}{2}$ para $\mathrm{SU}(\mathrm{N})$.

$$
\operatorname{Tr}\left[T^{a}, T^{b}\right]=C(r) \delta^{a b} .
$$

Chega-se à conclusão de que a amplitude invariante ao quadrado será descrita por:

$$
\begin{aligned}
\sum_{\text {spin,cor }}\left|\mathscr{M}_{\Gamma}\right|^{2}= & \frac{1}{3} \frac{1}{2} \operatorname{Tr}\left[\left(k+m_{q}\right) \gamma^{\mu}\left(g_{V}^{q}-g_{A}^{q} \gamma^{5}\right)\left(\not k^{\prime \prime}-m_{q}\right) \gamma^{v}\left(g_{V}^{q}-g_{A}^{q} \gamma^{5}\right)\right] \\
& \times\left(-g_{\mu \nu}+\frac{P^{\mu} P^{v}}{M_{G}^{2}}\right)
\end{aligned}
$$

com o fator $\frac{1}{3}$ vindo da soma do número de cor $N=3$. Restando apenas o cálculo para o traço das matrizes de Dirac em $k$, $k^{\prime \prime}$, sendo $k \equiv \gamma^{\mu} k_{\mu}$, com os traços de $\gamma^{\mu}$ bem conhecidos na literatura, por exemplo em [5]. O que resulta em:

$$
\begin{aligned}
\sum_{\text {spin,cor }}\left|\mathscr{M}_{\Gamma}\right|^{2}=\frac{1}{6} & {\left[4\left(\left(g_{V}^{q}\right)^{2}+\left(g_{A}^{q}\right)^{2}\right)\left(k_{\mu}^{\prime} k_{v}+k_{v}^{\prime} k_{\mu}-g_{\mu v}\left(k \cdot k^{\prime}\right)\right)+8 i g_{V}^{q} g_{A}^{q} \epsilon_{\mu \alpha \nu \beta} k^{\prime \alpha} k^{\beta}\right.} \\
& \left.-4 m_{q}^{2}\left(\left(g_{V}^{q}\right)^{2}-\left(g_{A}^{q}\right)^{2}\right) g_{\mu v}\right] \times\left(-g_{\mu v}+\frac{P^{\mu} P^{v}}{M_{G}^{2}}\right)
\end{aligned}
$$

Finalmente, ao contrair os termos da amplitude, é obtido o resultado em função dos quadrimomentos de cada quark $\mathrm{k}$ e k':

$$
\left|\mathscr{M}_{\Gamma}\right|^{2}=\frac{2}{3}\left[\left(\left(g_{V}^{q}\right)^{2}+\left(g_{A}^{q}\right)^{2}\right)\left(k^{\prime} \cdot k+\frac{2}{M^{2}}(k \cdot P)\left(k^{\prime} \cdot P\right)+3 m_{q}^{2}\left(\left(g_{V}^{q}\right)^{2}-\left(g_{A}^{q}\right)^{2}\right)\right] .\right.
$$

Considerando o sistema no centro de massas é possível redefinir os quadrimomentos em função da massa do primeiro modo de excitado do bóson de gauge $M_{G}$ e o momento vetorial dos quarks iniciais $\mathrm{k}$. Assim o sistema será descrito por:

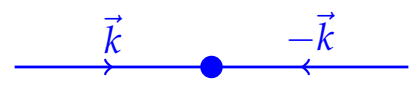




$$
\begin{gathered}
P=\left(M_{G}, 0\right) ; \\
k=\left(\frac{M_{G}}{2}, \vec{k}\right) ; \\
k^{\prime}=\left(\frac{M_{G}}{2},-\vec{k}\right) ; \\
|\vec{k}|=\sqrt{\left(\frac{M_{G}}{2}\right)^{2}-m_{q}^{2} .}
\end{gathered}
$$

Nota-se que os produtos entre os quadrimomentos são dados em função das massas do bóson de gauge $M_{G}$ e dos quarks $m_{q}$ :

$$
\begin{gathered}
\left(k \cdot k^{\prime}\right)=\frac{M_{G}^{2}}{2}-m_{q}^{2} ; \\
(k \cdot P)=\left(k^{\prime} \cdot P\right)=\frac{M_{G}^{2}}{2} .
\end{gathered}
$$

Substituindo esses valores dos produtos escalares na amplitude

$$
\left|\mathscr{M}_{\Gamma}\right|^{2}=\frac{2}{3}\left[\left(\left(g_{V}^{q}\right)^{2}+\left(g_{A}^{q}\right)^{2}\right)\left(M_{G}^{2}-m_{q}^{2}\right)+3 m_{q}^{2}\left(\left(g_{V}^{q}\right)^{2}-\left(g_{A}^{q}\right)^{2}\right)\right] .
$$

Ao substituir o valor da amplitude descrita acima (4.1.1.13) na Largura descrita pela equação (4.1.1.2) resulta em:

$$
\Gamma=\sum_{q} \frac{M_{G}}{24 \pi} \sqrt{1-\frac{4 m_{q}^{2}}{M_{G}^{2}}}\left[\left(g_{V}^{q}\right)^{2}+\left(g_{A}^{q}\right)^{2}+\left(2\left(g_{V}^{q}\right)^{2}-4\left(g_{A}^{q}\right)^{2}\right) \frac{m_{q}^{2}}{M_{G}^{2}}\right] .
$$

\subsubsection{Seção de Choque Partônica}

Será apresentado o cálculo da seção de choque partônica de produção do bóson de gauge a partir de dois quarks $q \bar{q} \rightarrow G^{(1)} \rightarrow q^{\prime} \bar{q}^{\prime}$. Novamente, a Lagrangiana que descreve a interação entre os quarks e o primeiro modo excitado do bóson de gauge será dada por uma interação $S U(3)$ descrita na equação (4.1.1.1). Será considerado o decaimento em quarks no cálculo da seção de choque apresentada, no qual foi escolhido os estados finais em quarks top para simplificar a notação.

$$
q \bar{q} \rightarrow G_{\mu}^{(1)} \rightarrow t \bar{t}
$$

Logo, o diagrama de Feynman para o processo estudado será no canal s, o que está representado abaixo: 


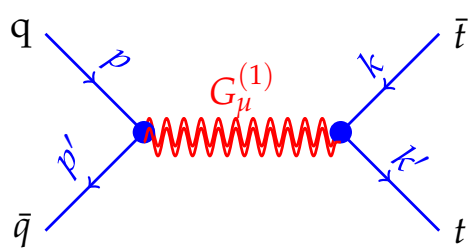

Sendo $\mathrm{p}, \mathrm{p}^{\prime}, \mathrm{k}^{\prime}, \mathrm{k}$ os quadrimomentos de seus respectivos quarks e antiquarks $\mathrm{q}, \bar{q}, \mathrm{t}$, $\bar{t}$. Logo, dado os propagadores para o diagrama acima, a amplitude invariante pode ser calculada para escrever a seção de choque partônica para este processo. Deste modo, foi utilizado o propagador do primeiro modo excitado do bóson de gauge, de massa $M_{G}$ e quadrimomento $P_{\mu}$. Representado pelo propagador:

\section{$G_{u}^{(1)}$

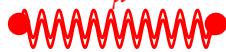

O que é correspondente a:

$$
\frac{-i}{P^{2}-M_{G}^{2}+i M_{G} \Gamma_{G}}\left(g_{\mu v}-\frac{P_{\mu} P_{v}}{M_{G}^{2}}\right) \delta_{a b},
$$

com $\Gamma_{G}$ a largura (4.1.1.14), calculada na seção anterior. Então, a amplitude invariante é descrita por:

$$
\begin{aligned}
i \mathscr{M}_{G}= & \bar{v}\left(p^{\prime}\right)_{i}(-i) T_{i j}^{a} \gamma^{\mu}\left(g_{V}^{q}-g_{A}^{q} \gamma^{5}\right) u(p)_{j} \frac{-i}{\left(p+p^{\prime}\right)^{2}-M_{G}^{2}+i M_{G} \Gamma_{G}} \\
& \bar{u}(k)_{i^{\prime}}(-i) T_{i^{\prime} j^{\prime}}^{a} \gamma^{\mu}\left(g_{V}^{t}-g_{A}^{t} \gamma^{5}\right) v\left(k^{\prime}\right)_{j^{\prime}} .
\end{aligned}
$$

Calcula-se o módulo ao quadrado da amplitude, tomando a média dos valores dos números quânticos spin e cor. Além de somar sob os estados finais, obtendo a amplitude ao quadrado:

$$
\begin{aligned}
& \sum_{\text {spin,cor }}\left|\mathscr{M}_{G}\right|^{2}=\frac{1}{4} \frac{1}{N^{2}} \frac{T_{i i^{\prime}}^{a} T_{j j^{\prime}}^{a} T_{k k^{\prime}}^{b} T_{l l^{\prime}}^{b}}{\left|\left(p+p^{\prime}\right)^{2}-M_{G}^{2}+i M_{G} \Gamma_{G}\right|^{2}} \\
& \times\left[\bar{v}\left(p^{\prime}\right)_{i}^{s} \gamma^{\mu}\left(g_{V}^{q}-g_{A}^{q} \gamma^{5}\right) u(p)_{i^{\prime}}^{s^{\prime}}\right]\left[\bar{u}(p)_{l^{\prime}}^{s^{\prime}} \gamma_{v}\left(g_{V}^{q}-g_{A}^{q} \gamma^{5}\right) v\left(p^{\prime}\right)_{l}^{s}\right] \\
& \times\left[\bar{v}\left(k^{\prime}\right)_{k^{\prime}}^{r^{\prime}} \gamma^{v}\left(g_{V}^{t}-g_{A}^{t} \gamma^{5}\right) u(k)_{k}^{r}\right]\left[\bar{u}(k)_{j}^{r} \gamma_{\mu}\left(g_{V}^{t}-g_{A}^{t} \gamma^{5}\right) v\left(k^{\prime}\right)_{j^{\prime}}^{r^{\prime}}\right],
\end{aligned}
$$

com $N=3$ número de cor, e o fator $\frac{1}{4}$ vindo da média da soma de spin. Os traços dos geradores do grupo $S U(3)$ obedecem à álgebra (4.1.1.9), que resulta em:

$$
\operatorname{Tr}\left[T^{a} T^{b}\right] \operatorname{Tr}\left[T^{a} T^{b}\right]=\frac{1}{4} \delta^{a b} \delta^{a b}=\frac{1}{4}\left(N^{2}-1\right) .
$$


Dadas as relações de completeza (4.1.1.7), pode-se escrever a amplitude ao quadrado em função das massas e quadrimomentos das partículas envolvidas no processo analisado:

$$
\begin{aligned}
\sum_{\text {spin,cor }}\left|\mathscr{M}_{G}\right|^{2}= & \frac{1}{4} \frac{1}{N^{2}} \frac{1}{4}\left(N^{2}-1\right) \frac{1}{\left(\left(p+p^{\prime}\right)^{2}-M_{G}^{2}\right)^{2}+M_{G}^{2} \Gamma_{G}^{2}} \\
& \operatorname{Tr}\left[\left(\not p^{\prime}-m_{q}\right) \gamma^{\mu}\left(g_{V}^{q}-g_{A}^{q} \gamma^{5}\right)\left(p+m_{q} \gamma_{v}\left(g_{V}^{q}-g_{A}^{q} \gamma^{5}\right)\right]\right. \\
& \operatorname{Tr}\left[\left(k+m_{t}\right) \gamma_{\mu}\left(g_{V}^{t}-g_{A}^{t} \gamma^{5}\right)\left(\not k^{\prime \prime}-m_{q} \gamma^{v}\left(g_{V}^{t}-g_{A}^{t} \gamma^{5}\right)\right] .\right.
\end{aligned}
$$

O cálculo dos traços das matrizes de Dirac resulta em:

$$
\begin{aligned}
&\left|\overline{\mathscr{M}}_{G}\right|^{2}= \frac{1}{18} \\
&\left(\left(p+p^{\prime}\right)^{2}-M_{G}^{2}\right)^{2}+M_{G}^{2} \Gamma_{G}^{2} \\
& {\left[4\left(\left(g_{V}^{q}\right)^{2}+\left(g_{A}^{q}\right)^{2}\right)\left(p^{\prime \mu} p^{v}+p^{\prime \nu} p^{\mu}-g^{\mu \nu}\left(p^{\prime} \cdot p\right)+8 i g_{V}^{q} g_{A}^{q} \epsilon^{\mu \beta v \alpha} p_{\beta}^{\prime} p_{\alpha}\right]\right.} \\
& {\left[4 ( ( g _ { V } ^ { t } ) ^ { 2 } + ( g _ { A } ^ { t } ) ^ { 2 } ) \left(k_{\mu}^{\prime} k_{v}+k_{v}^{\prime} k_{\mu}-g^{\mu v}\left(k^{\prime} \cdot k\right)+8 i g_{V}^{t} g_{A}^{t} \epsilon_{\mu \beta \nu \alpha} k_{\beta}^{\prime} k_{\alpha}-\right.\right.} \\
&\left.-4 m_{t}^{2}\left(\left(g_{V}^{t}\right)^{2}-\left(g_{A}^{t}\right)^{2}\right) g_{\mu \nu}\right] .
\end{aligned}
$$

Ao contrair os índices dos termos obtidos do cálculo dos traços, observa-se que a amplitude depende do produto escalar dos momentos de cada partícula:

$$
\begin{aligned}
\left|\overline{\mathscr{M}}_{G}\right|^{2}= & \frac{1}{18} \times 16 \frac{1}{\left(\left(p+p^{\prime}\right)^{2}-M_{G}^{2}\right)^{2}+M_{G}^{2} \Gamma_{G}^{2}} \\
& {\left[2\left(\left(g_{V}^{q}\right)^{2}+\left(g_{A}^{q}\right)^{2}\right)\left(\left(g_{V}^{t}\right)^{2}+\left(g_{A}^{t}\right)^{2}\right)\left(\left(p^{\prime} \cdot k^{\prime}\right)(p \cdot k)+\left(p^{\prime} \cdot k\right)\left(p \cdot k^{\prime}\right)\right)+\right.} \\
& +2 m_{t}^{2}\left(\left(g_{V}^{t}\right)^{2}-\left(g_{A}^{t}\right)^{2}\right)\left(\left(g_{V}^{t}\right)^{2}+\left(g_{A}^{t}\right)^{2}\right)\left(p^{\prime} \cdot p\right) \\
& -8 g_{V}^{q} g_{A}^{q} g_{V}^{t} g_{V}^{t}\left[\left(p^{\prime} \cdot k^{\prime}\right)(p \cdot k)-\left(p^{\prime} \cdot k\right)\left(p \cdot k^{\prime}\right)\right] .
\end{aligned}
$$

Logo, é possível analisar o problema no centro de momentos, tal que a seção de choque seja escrita em termos observáveis. Assim serão obtidas relações entre os quadrimomentos e energia do centro de massas do processo, além das massas de cada partícula. O referencial escolhido será representado no esquema abaixo:

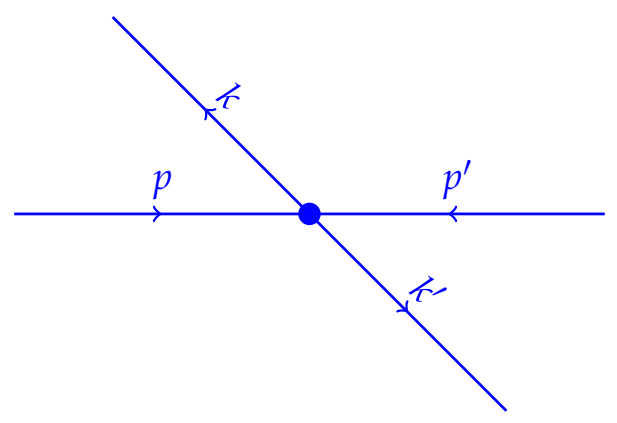




$$
\begin{gathered}
p=(E, 0,0, E) ; \\
p^{\prime}=(E, 0,0,-E) ; \\
k=\left(E, \sqrt{E^{2}-m_{t}^{2}} \vec{k}\right) ; \\
k^{\prime}=\left(E, \sqrt{E^{2}-m_{t}^{2}} \vec{k}^{\prime}\right) .
\end{gathered}
$$

É possível também definir a variável de Mendelstam $\hat{s}=4 E^{2}$, onde E é a energia partônica no centro de massas, além do parâmetro $c=\left(\sqrt{1-\frac{m_{t}^{2}}{E^{2}}}\right) \cos \theta$. Deste modo, os produtos escalares são dados em função da energia do centro de massas, representada por $\hat{\mathrm{s}}$, e do ângulo de espalhamento $\theta$.

$$
\begin{gathered}
p^{\prime} \cdot p=k^{\prime} \cdot k=2 E^{2}=\frac{\hat{s}}{2} ; \\
p^{\prime} \cdot k^{\prime}=p \cdot k=E^{2}-E^{2} c=\frac{\hat{s}}{4}(1-c) ; \\
p^{\prime} \cdot k=p \cdot k^{\prime}=E^{2}+E^{2} c=\frac{\hat{s}}{4}(1+c) .
\end{gathered}
$$

Finalmente, os valores dos produtos escalares são substituídos na amplitude invariante:

$$
\begin{aligned}
\left|\overline{\mathscr{M}}_{G}\right|^{2}=\frac{2}{9} & \frac{\hat{\mathrm{s}}^{2}}{\left((\hat{\mathbf{s}})^{2}-M_{G}^{2}\right)^{2}+M_{G}^{2} \Gamma_{G}^{2}} \\
& \times\left[\left(\left(g_{V}^{q}\right)^{2}+\left(g_{A}^{q}\right)^{2}\right)\left(\left(g_{V}^{t}\right)^{2}+\left(g_{A}^{t}\right)^{2}\right)\left(1+c^{2}\right)+4 \frac{m_{t}^{2}}{s}\left(\left(g_{V}^{t}\right)^{2}-\left(g_{A}^{t}\right)^{2}\right)\left(\left(g_{V}^{t}\right)^{2}+\left(g_{A}^{t}\right)^{2}\right)\right. \\
& \left.-8 g_{V}^{q} g_{A}^{q} g_{V}^{t} g_{A}^{t} c\right]
\end{aligned}
$$

o que resulta na amplitude em função dos acoplamentos, massas, largura e energia do centro de massas.A partir dessa expressão é possível calcular a seção de choque em função de $M_{G^{(1)}}$ e do número de sítios $\mathrm{N}$, o que caracteriza os acoplamentos $g_{V}$ e $g_{A}$.

$$
\frac{d \sigma}{d \Omega}=\frac{1}{2 E_{A} E_{B}} \frac{1}{\left|V_{A}^{2}-V_{B}^{2}\right|} \frac{p_{\mu}}{16 \pi^{2} E_{C M}}\left|\bar{M}_{G}\right|^{2}
$$

Ao calcular o espaço de fases:

$$
\frac{d \sigma}{d \Omega}=\frac{1}{E_{C M}^{2}} \frac{1}{64 \pi^{2}} \sqrt{1-4 \frac{m_{q}^{2}}{M_{G}^{2}}}\left|\overline{\mathscr{M}}_{G}\right|^{2} .
$$

Portanto, obtém-se a seção de choque partônica para uma certo valor de ŝ com o propósito de encontrar vínculos para a massa do modo excitado do bóson de gauge dependendo do acoplamento, ou seja, do número de sítios no quiver. 


\subsection{Seção de Choque $p p \rightarrow G^{(1)} \rightarrow q \bar{q}$}

Esta seção tem por objetivo descrever como foram obtidos os valores das seções de choque do decaimento do primeiro modo excitado do bóson de gauge, o que será demonstrado a partir da seção de choque partônica calculada até agora em (4.1.2.7). A fim de comparar o resultado com dados obtidos pelo LHC, as seções de choque devem descrever colisões entre dois prótons com a energia do centro de massas a $8 \mathrm{TeV}$. Logo, deve-se apresentar as seções de choque dos processos de colisões de dois prótons, o que dará lugar à interação intermediadas pelo primeiro estado excitado do octeto ou singleto de cor.

$$
p p \rightarrow G^{(1)} \rightarrow q \bar{q}
$$

Logo, as seções de choque partônicas calculadas serão convoluídas com funções de distribuição ou PDF, sendo então possível estudar a fenomenologia das Teorias Quiver. Deste modo, serão calculadas seções de choque para diversos valores de $\mathrm{N}$ sítios, em diferentes canais do LHC de procura por nova física. Esta fenomenologia será feita ao utilizar os acoplamentos resultantes da teoria desconstruída em poucos sítios demonstrados no capítulo 3.

É apresentado o cálculo para a seção de choque partônica de processos mediados pelo estado excitado do glúon $G^{(1)}$. Entretanto, é necessário haver interação eletrofraca no Quiver. Também é estudada as seções de choque partônicas vindas das interações dos bósons neutros $\gamma^{\prime}$ e $Z^{\prime}$. Esses cálculos são análogos, alterando apenas os valores para os acoplamentos, e considerando os bósons eletrofracos sem cor. O cálculo das seções de choque próton-próton foram feitos com o Mathematica Wolfram, além de usar alguns pacotes específicos desse programa. Os acoplamentos obtidos para as Teorias Quiver foram apresentados no capítulo 3. Além disso, o acoplamento $\alpha$ é avaliado na energia da colisão. Para isto foi utlizado o pacote RunDec [39] do Mathematica, que o calcula a 2 loops da função $\beta$.

\subsubsection{Funções de Distribuição Partônica}

O LHC consta de 2 feixes de prótons com energia do centro de massas fixa a um dado $\sqrt{s}$. Para calcular seções de choque no LHC a partir de seções de choque partônicas é preciso obter a probabilidade de um parton dentro do próton com energia $\sqrt{s}$ ter um dado momento. Essas probabilidades são as chamadas Funções de Distribuições Partônicas (PDF). Elas podem ser estudadas com detalhe nas referências [40] e [5]. Deste modo, a partir da seção de choque partônica calculada na seção anterior (4.1.2.7), integrada sob o ângulo sólido, pode-se inferir a contribuição de cada quark para o processo:

$$
p+p \rightarrow G^{(1)} \rightarrow t \bar{t}
$$

As funções de distribuição descrevem a estrutura do próton, ou seja, a probabilidade de se encontrar algum constituinte desse. É certo que cada próton possui dois quarks 
up e um down de valência, além da presença de glúons. Entretanto, como se tratam de colisões a altas energias, é possível que haja também criação de pares, então devese considerar a probabilidade de haver interação entre todos os diversos quarks do chamado mar. Tais partículas vêm de flutuações do vácuo, sendo necessário levar em consideração o cálculo de pares de quarks e glúons vindos do mar.

Para um próton com quadrimomento $\mathrm{P}$, o quark que dará lugar à interação na colisão terá uma fração deste quadrimomento $\mathrm{xP}$, onde $0 \leq x \leq 1$. Portanto, é possível descrever a distribuição de cada quark como uma função da fração do momento $\mathrm{x}$, a qual também dependerá da energia do centro de massas, $f\left(x, z \times E_{C M}\right)$, com $0 \leq z \leq 1$. Também é importante ressaltar que elas serão normalizadas respeitando as proporções encontradas no próton entre up e down, ou seja a contribuição de um será o dobro do outro. Logo, deve-se respeitar as distribuições de partículas elementares no proton, por isso, as funções de distribuição devem ter a seguinte normalização:

$$
\begin{gathered}
\int_{0}^{1}\left[f_{u}(x)-f_{\bar{u}}(x)\right]=2, \int_{0}^{1}\left[f_{d}(x)-f_{\bar{d}}(x)\right]=1 \\
\int_{0}^{1} d x x\left[f_{u}(x)+f_{d}(x)+f_{\bar{u}}(x)+f_{\bar{d}}(x)+f_{g}(x)\right]=1 .
\end{gathered}
$$

O processo em foco neste trabalho será produção de nova física no canal s a partir da colisão de dois prótons com uma dada energia do centro de massas. A fim de calcular a seção de choque partônica do processo desejado, integra-se a seção de choque que descreve a interação mediada pelo primeiro modo excitado do bóson de gauge, a qual será multiplicada pelas distribuições partônicas correspondentes a cada próton em questão. São integrados ambos parâmetros x e z, que correspondem respectivamente à fração do quadrimomento total do próton e a fração da energia do centro de massas que cada constituinte pode obter. Deve-se também somar a contribuição de cada quark para o processo final.

$$
\sigma\left(p p \rightarrow q_{r} \bar{q}_{r}\right)=\sum_{q} \int_{z}^{1} d x \int_{0}^{1} d z \frac{1}{x} f_{q}\left(x, z E_{C M}\right) f_{\bar{q}}\left(\frac{z}{x}, z E_{C M}\right) \sigma\left(q \bar{q}_{r} \rightarrow q \bar{q}_{r}\right) .
$$

Finalmente, a seção de choque depende da energia do centro de massas, valor da massa do estado excitado dos bósons de gauge e o valor dos seus acoplamentos do primeiro estado excitado do glúon com os quarks do Modelo Padrão. Logo, a fenomenologia da teoria desconstruida em um dado número de sítios será gerada a partir destes parâmetros. É possível deduzir os vínculos de massa para novos bósons de gauge ao comparar as seções de choque com os experimentos de colisão entre prótons produzidos no LHC. 

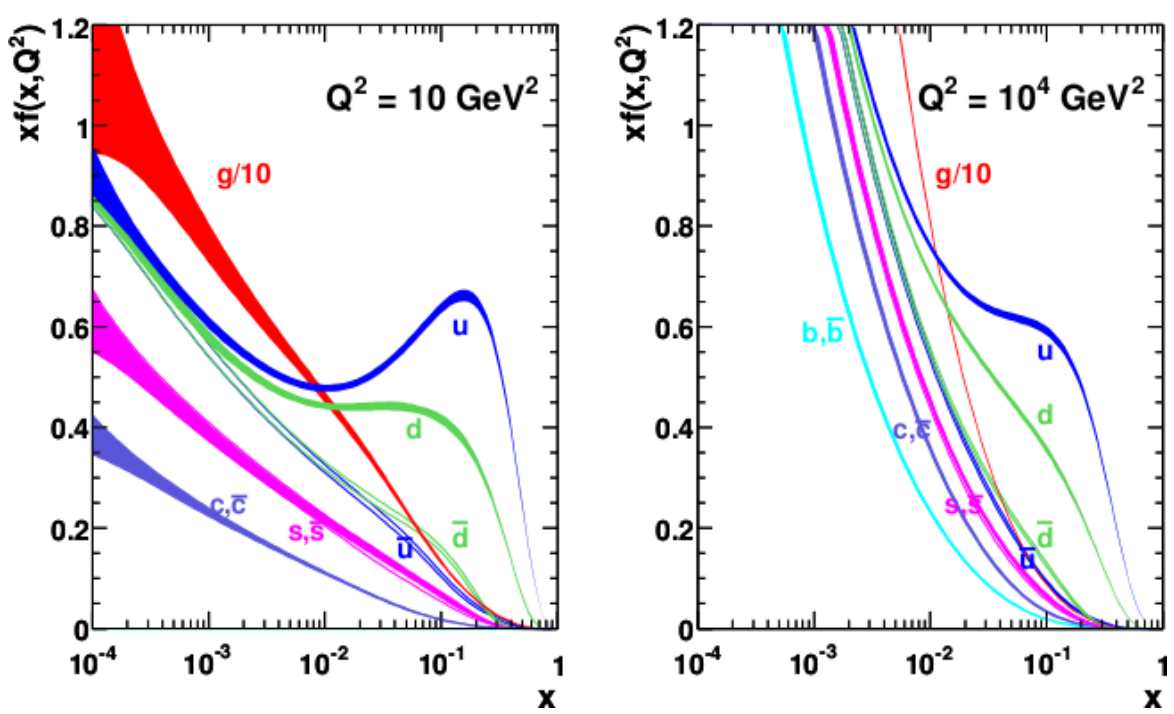

Figura 4.2: Funções de distribuição partônicas $x f(x)$ a $10 \mathrm{GeV}^{2}$ e $10^{4} \mathrm{GeV}^{2}$ calculadas a um loop pela PDF mstw [41]

As PDF são obtidas a partir de dados experimentais vindos de diversos experimentos a uma dada energia. No entanto, é desejado obter essas funções a energias mais altas, no caso a escala TeV explorada pelo LHC. Logo, deve-se convoluir essas medições através das equações de Altarelli-Parisi , também descritas em detalhes nas referências [40] e [5]. Nesse trabalho foi utilizado o pacote do Mathematica mstwpdf.m [41] para obter as funções de distribuição partônicas. A figura 4.2 representa as distribuições de cada constituinte dos prótons a uma dada energia e sua convolução na PDF escolhida mstw. Apesar de existir um conjunto de diferentes programas numéricos que calculam tais funções, o pacote mstw foi escolhido por descrever com precisão suficiente a análise feita da fenomenologia da Teoria Quiver.

\subsection{Procura no LHC}

Os vínculos experimentais do modelo foram retirados de dados obtidos pelo LHC, apresentados até então pelas colaborações CMS e ATLAS na procura do decaimento das partículas de interesse. Neste trabalho foram analisados eventos em canais de produção em dijatos [3], pares de quark top [4] e bottom [2] para energia do centro de massas equivalente a $\sqrt{s}=8 \mathrm{TeV}$, com luminosidade integrada de $19.6 \mathrm{fb}^{-1}$ para o CMS e $14.5 \mathrm{fb}^{-1}$ para o ATLAS.

Os dados experimentais são apresentados como, por exemplo, na figura 4.3, a qual foi obtida pela colaboração CMS na produção de dijatos em [3]. Os dados analisados são apresentados como a seção de choque vezes a razão de ramificação em função do valor da massa da ressonância possivelmente produzida pelo experimento. Os 

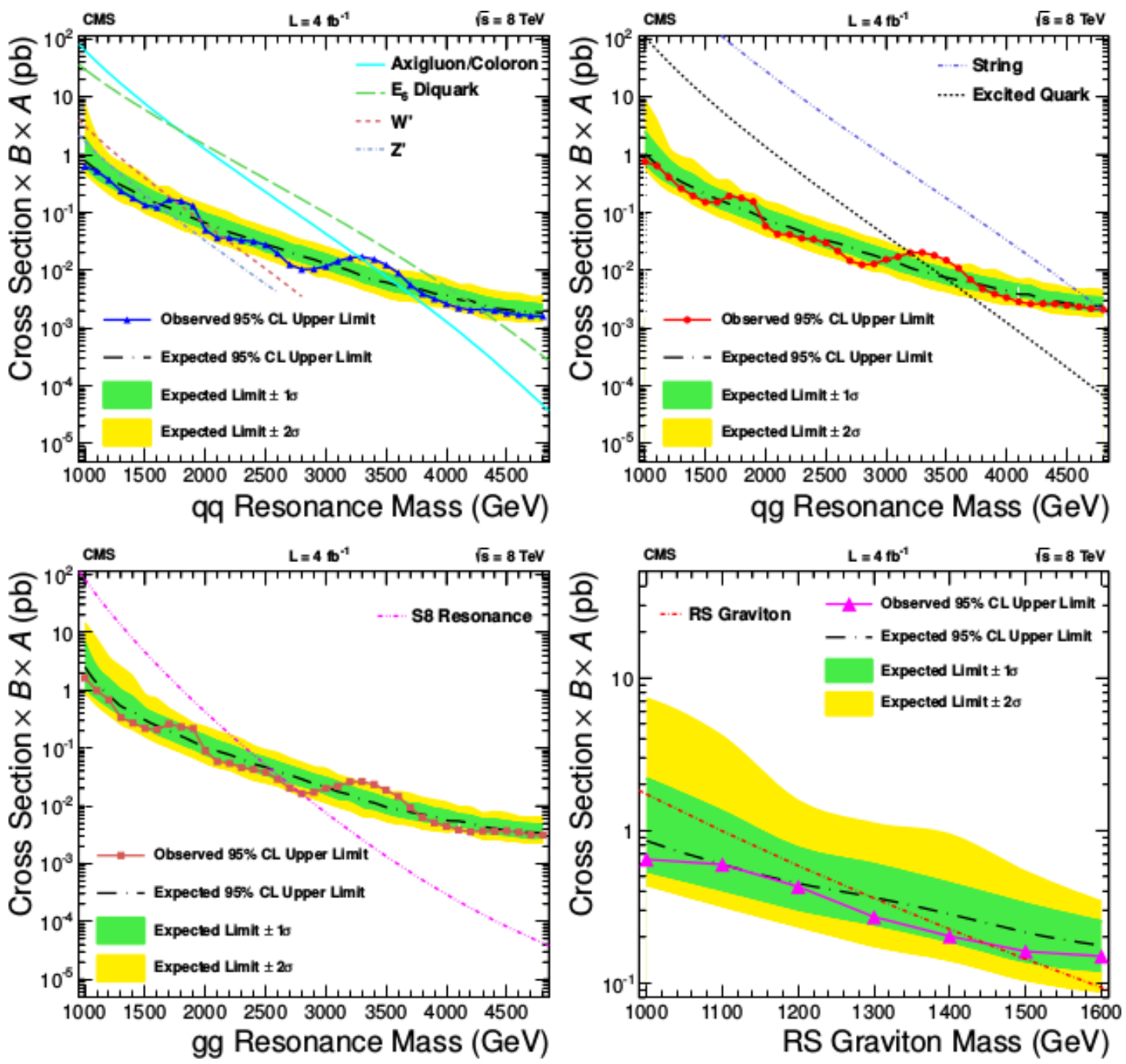

Figura 4.3: Seção de choque vezes a razão de ramificação em função do valor da massa da ressonância, a qual está multiplicada pela Acceptance $A=0.6$. Os pontos representam vínculos observados a 95\% de nível de confiança. As bandas correspondem aos vínculos esperados de $1 \sigma$ e $2 \sigma$ de $\sigma \times B r$. Além disso, são ilustradas previsões teórias de outras teorias além do MP, representadas pelas curvas diagonais aos dados. Dados apresentados pela colaboração CMS na produção de dijatos a $\sqrt{s}=8 \mathrm{TeV}$ na referência [3]. 
pontos correspondem aos vínculos observados com 95\% de nível de confiança. Os vínculos de massa são obtidos ao comparar os cálculos da seção de choque de decaimento do primeiro modo excitado dos bósons de gauge nas Teorias Quiver.

A busca por física exótica é feita através de um mecanismo estatístico chamado "Bump Searches". Nele, a teoria já estabelecida pelo MP é usada como fundo. Uma simulação da QCD gera o fundo que é simulado e subtraído da seção de choque observada. Logo, o resultado apresenta a seção de choque vezes a razão de ramificação a uma dada energia do centro de massas. Esses são calculados para cada valor de massa da ressonância e subtraído da hipótese inicial gerada pela QCD. A partir desses dados, as buscas por nova física são feitas ao analisar cada intervalo (bin) de massas, observando se há excessos ou faltas no fundo previsto [4] [42].

Além disso, ao tratar partículas carregadas pelo índice de cor deve-se considerar o termo de interferência com a QCD na seção de choque. No entanto, tendo o mecanismo de procura em vista, esse termo de interferência deve ser desprezado, pois ele é subtraído junto com o fundo da QCD.

\subsection{Resultados em $\sqrt{s}=8 \mathrm{TeV}$}

Finalmente, são apresentados os resultados numéricos obtidos para a colisão de dois prótons com $\sqrt{s}=8 \mathrm{TeV}$. Sendo que as seções de choque totais da produção e decaimento da primeira ressonância do octeto de cor e eletrofraca foram calculados para produção de jatos, quarks top e bottom. Utiliza-se os acoplamentos já calculados pelo grupo para 5, 10 e 16 sítios ( $\mathrm{N}=4,9$ e 15).

As figuras 4.4, 4.5 e 4.6 traçam a seção de choque multiplicada pela razão de ramificação em função da massa do primeiro modo excitado do glúon. As figuras representam a produção de quarks leves ou jatos, pares de top e bottom, respectivamente. Além disso, experimentalmente foi eliminada a possibilidade da ressonância obter massa menor que $500 \mathrm{GeV}$, também foram analisados dados até aproximadamente $4 \mathrm{TeV}$ [3] [4] [2] . Portanto, são analisados valores para $500 \mathrm{GeV} \leq M_{G} \leq 5 \mathrm{TeV}$.

Analogamente, foram calculadas as seções de choque vezes a razão de ramificação para o singleto de cor, sendo este o primeiro modo excitado do fóton e do bóson de gauge $Z$. As contribuições dos dois bósons $\left(Z^{\prime}+\gamma^{\prime}\right)$ estão somadas nas figuras apresentadas, pois os dois estados são degenarados. Assim sendo, será difícil detectá-los separadamente. Novamente são demonstrados os cálculos para produção de quarks leves na figura 4.7, pares de top no gráfico 4.8 e bottom em 4.9 .

Observa-se que a seção de choque multiplicada pela razão de ramificação $(\sigma \times B R)$ diminui a medida que o número de sítios cresce, tanto para o octeto quanto para o singleto de cor. Este comportamento é esperado, pois o acoplamento dos quarks leves ao primeiro estado excitado do boson de gauge é menor quanto maior o valor de N, ou seja quanto mais próximo do limite do contínuo, o qual leva ao caso extradimensional $A d S_{5}$. Por outo lado, os canais de decaimento em $t \bar{t}$ são muito mais degenerados, dado que os acoplamentos do quark top crescem com $\mathrm{N}$. 


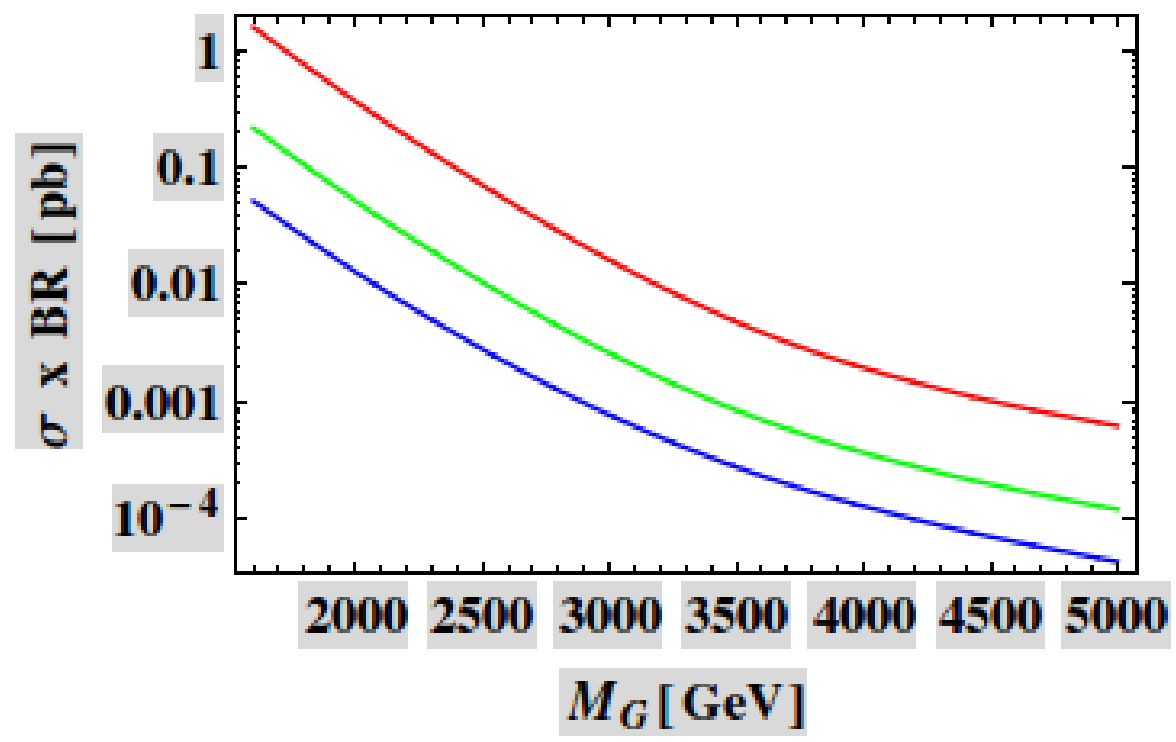

Figura 4.4: Seção de Choque multiplicada pela razão de ramificação em função da massa do primeiro estado excitado do glúon correspondente ao decaimento em dois jatos a $\sqrt{s}=8 \mathrm{TeV}$. As linhas vermelha, verde, azul correspondem, respectivamente ao caso com 5,10 e 16 sítios

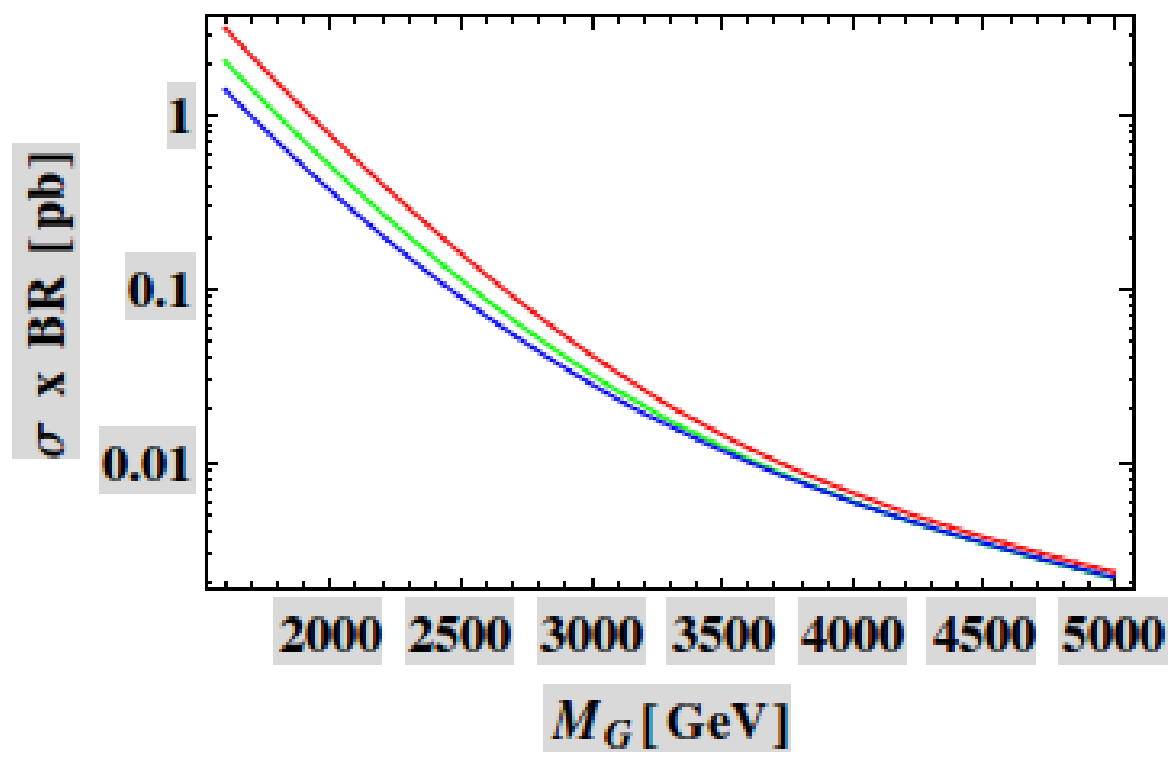

Figura 4.5: Seção de Choque multiplicada pela razão de ramificação em função da massa do primeiro estado excitado do glúon correspondente ao decaimento em $t \bar{t}$ a $\sqrt{s}=8 \mathrm{TeV}$. As linhas vermelha, verde, azul correspondem, respectivamente ao caso com 5,10 e 16 sítios 


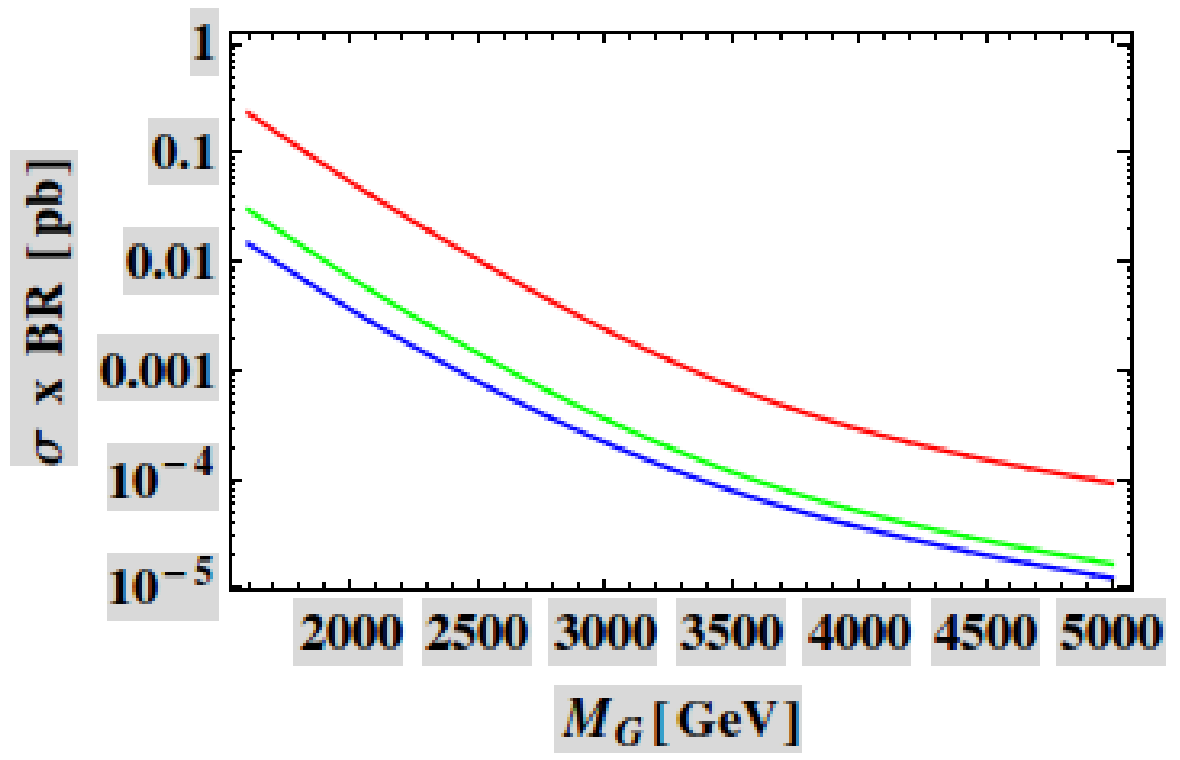

Figura 4.6: Seção de Choque multiplicada pela razão de ramificação em função da massa do primeiro estado excitado do glúon correspondente ao decaimento em $b \bar{b}$ a $\sqrt{s}=8 \mathrm{TeV}$. As linhas vermelha, verde, azul correspondem, respectivamente ao caso com 5,10 e 16 sítios

\subsection{Fenomenologia no LHC}

O objetivo desse trabalho é a análise fenomenológica das Teorias Quiver no LHC. Os dados experimentais foram discutidos na seção 4.3, deles são retirados vínculos que restringem o modelo e determinam sua validade. A procura por ressonâncias no LHC através de Bump Searches revela que a nova física pode ser encontrada como novas interações a partir de modos excitado dos bósons de gauge, como mostra na figura 4.3. Ou seja, um possível excesso localizado em um gráfico experimental determinará a massa da nova partícula. Os vínculos restringem os parâmetros da Teoria Quiver, ou seja, o número de sítios que determina os acoplamentos. Por isso, foram analisados processos para diferentes números de sítios, assim como apresentados na seção 4.4 .

\subsubsection{Vínculos experimentais}

Comparando os dados experimentais obtidos no LHC com os gráficos em $8 \mathrm{TeV}$ apresentados seção 4.4, são obtidos os vínculos para as massas do octeto e singleto de cor. Foram utilizados dados acumulados do LHC a $\sqrt{s}=8 \mathrm{TeV}$, com luminosidade integrada de $19.6 \mathrm{fb}^{-1}$ para o CMS e $14.5 \mathrm{fb}^{-1}$ para o ATLAS. Os vínculos das ressonâncias em dois jatos foram obtidas através de dados do CMS na referência [3]. Enquanto os vínculos obtidos pela colaboração ATLAS em processos de decaimento 


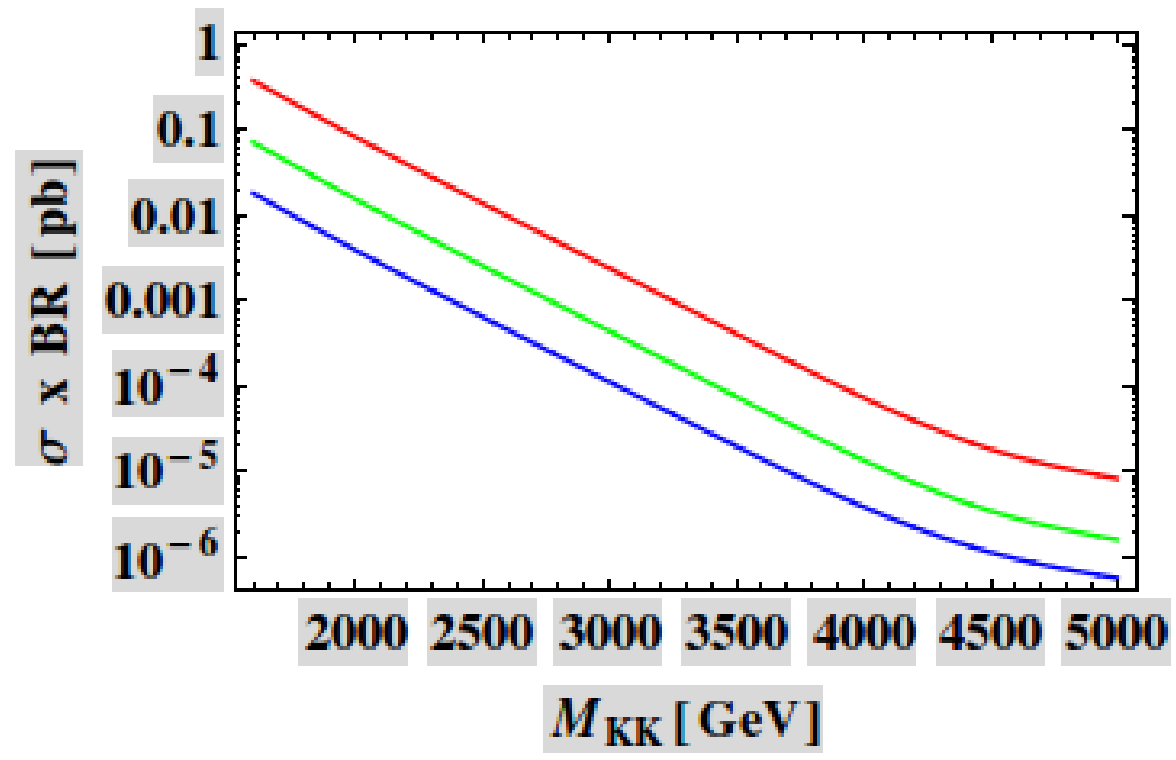

Figura 4.7: Seção de Choque multiplicada pela razão de ramificação em função da massa do primeiro estado excitado do $\left(Z^{\prime}+\gamma^{\prime}\right)$ correspondente ao decaimento em jatos a $\sqrt{s}=8 \mathrm{TeV}$. As linhas vermelha, verde, azul correspondem, respectivamente ao caso com 5,10 e 16 sítios

em $t \bar{t}$ foram obtidos em [4]. Além disso, os vínculos do CMS em $b \bar{b}$ foram consultados na referência [2].

\begin{tabular}{cccc}
\hline \hline \multicolumn{5}{c}{$\begin{array}{c}\text { Vínculos de Massa } \\
\text { Octeto de Cor (TeV) }\end{array}$} & & \\
\hline Sítios & $\mathbf{5}$ & $\mathbf{1 0}$ & $\mathbf{1 6}$ \\
\hline Jatos [3] & 3.0 & 1.6 & - \\
$b \bar{b}[2]$ & 1.6 & - & - \\
$t \bar{t}[4]$ & 2.7 & 2.6 & 2.5 \\
\hline \hline
\end{tabular}

Tabela 4.1: Vínculos diretos do LHC nas massas do octeto de cor das Teorias Quiver, com $\sqrt{s}=8 \mathrm{TeV}$ e luminosidade integrada de $19.6 \mathrm{fb}^{-1}$ para o CMS e $14.5 \mathrm{fb}^{-1}$ para o ATLAS.

Foi demonstrada na seção anterior 4.4 os resultados de $\sigma \times B r$ da interação do octeto de cor a 5, 10 e 16 sítios. Ao sobrepor cada curva sobre os dados experimentais em seus respectivos canais, retira-se o vínculo ao observar o ponto onde a teoria coincide com os pontos obtidos pelas colaborações do LHC, assim como discutido na figura 4.3. Deste modo, foram obtidos os vínculos para as massas do octeto de cor são apresentados na tabela 4.1 


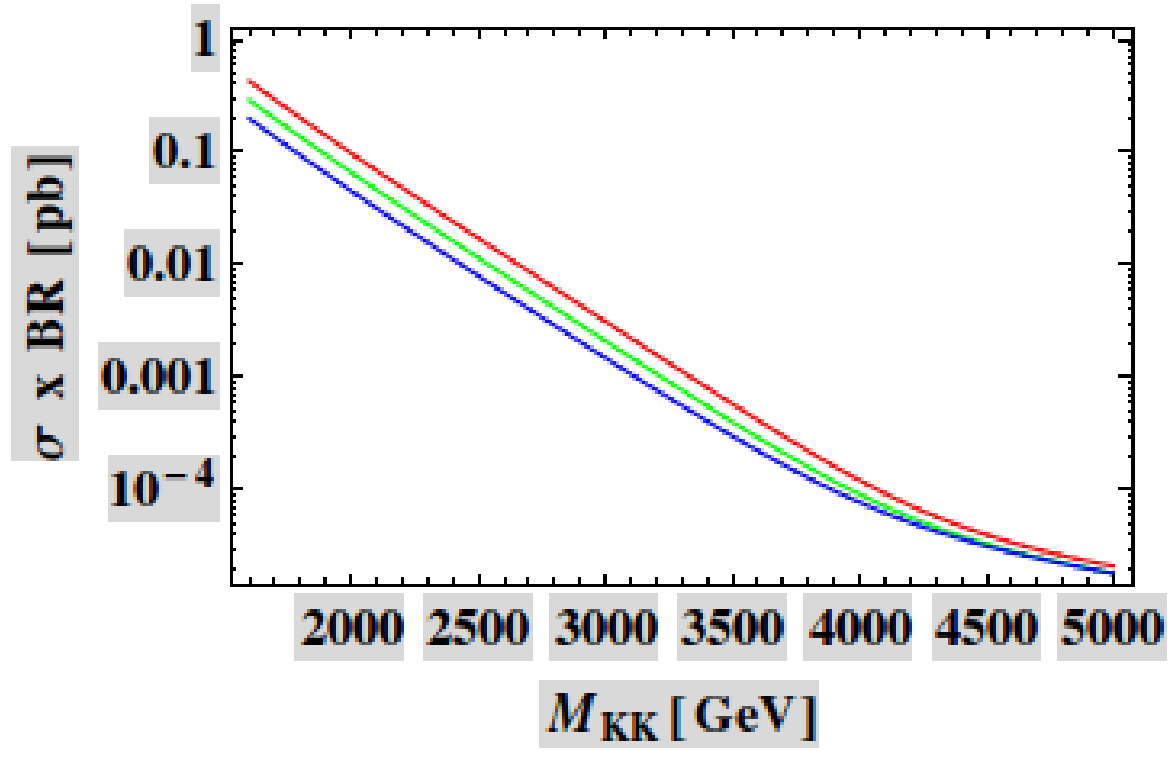

Figura 4.8: Seção de Choque multiplicada pela razão de ramificação em função da massa do primeiro estado excitado do $\left(Z^{\prime}+\gamma^{\prime}\right)$ correspondente ao decaimento em $t \bar{t}$ a $\sqrt{s}=8 \mathrm{TeV}$. As linhas vermelha, verde, azul correspondem, respectivamente ao caso com 5, 10 e 16 sítios

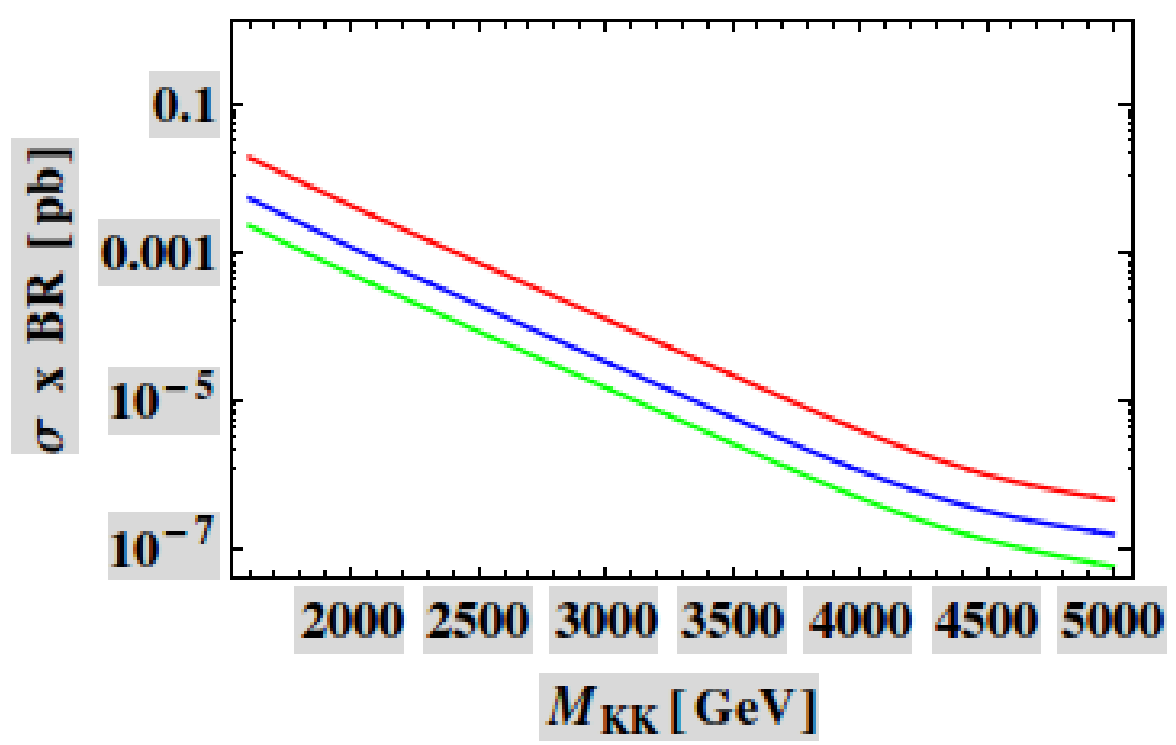

Figura 4.9: Seção de Choque multiplicada pela razão de ramificação em função da massa do primeiro estado excitado do $\left(Z^{\prime}+\gamma^{\prime}\right)$ correspondente ao decaimento em $b \bar{b}$ a $\sqrt{s}=8 \mathrm{TeV}$. As linhas vermelha, verde, azul correspondem, respectivamente ao caso com 5,10 e 16 sítios 
Observa-se que para o canal de decaimento em $t \bar{t}$ os vínculos não são modificados para diferentes valores de $\mathrm{N}$. O modo de decaimento em jatos é competitivo, sendo o caso a 5 sítios o melhor vínculo. De acordo com [25], os vínculos obtidos de experimentos de precisão eletrofraca são tipicamente na ordem de $3 \mathrm{TeV}$, o que se assemelha com os valores obtidos na Tabela 4.1. Entretanto, os resultados para $b \bar{b}$ não são significantes para esta energia.

\begin{tabular}{cccc}
\hline \hline \multicolumn{5}{c}{$\begin{array}{c}\text { Vínculos de Massa } \\
\left(Z^{\prime}+\gamma^{\prime}\right)(\mathrm{TeV})\end{array}$} \\
\hline Sites & $\mathbf{5}$ & $\mathbf{1 0}$ & $\mathbf{1 6}$ \\
\hline Dijet [3] & 1.7 & - & - \\
$b \bar{b}[2]$ & - & - & - \\
$t \bar{t}[4]$ & 2.1 & 2.0 & 1.8 \\
\hline \hline
\end{tabular}

Tabela 4.2: Vínculos diretos do LHC nas massas do singleto de cor das Teorias Quiver, com $\sqrt{s}=8 \mathrm{TeV}$ e luminosidade integrada de $19.6 \mathrm{fb}^{-1}$ para o CMS e $14.5 \mathrm{fb}^{-1}$ para o ATLAS.

Para os singletos de cor, apresentados na tabela 4.2, novamente observa-se que os vínculos no decaimento em $t \bar{t}$ não depedem significamente de $\mathrm{N}$, e são mais restringentes do que no caso de jatos. Esses também demonstram que o modelo é competitivo para pequenos números de sítios. Para $b \bar{b}$ os vínculos diretos também não são significantes a essa energia.

Em ambas as Tabelas 4.14.2, os elementos com um traço significam que os vínculos são muito baixos para serem consistentes com os limites de sabor e eletrofraco. De modo geral, os valores obtidos dos vínculos de massa estão abaixo do necessário para passar o vínculo de violação de sabor desses modelos $\simeq 3 \mathrm{TeV}$ [25]. No entanto, eles serão testados na próxima etapa do LHC, aumentando a energia e luminosidade.

\subsubsection{Perspectivas no LHC}

Foram apresentados vínculos de detecção direta na massa do primeiro estado excitado dos bósons de gauge da Teoria Quiver. Os vínculos de precisão eletrofracca são mais restritos do que os de procura direta, sendo ele aproximadamente $3 \mathrm{TeV}$ [25]. Enquanto os vínculos de sabor são um pouco mais restritos que os do LHC. Deve-se analisar dados a maior energia e luminosidade para obter vínculos mais restringentes, maiores que $3 \mathrm{TeV}$.

Até então foram obtidos dados com a energia do centro de massas de no máximo $\sqrt{s}=8 \mathrm{TeV}$ e luminosidade integrada a $19.6 \mathrm{fb}^{(-1)}$. Entretanto, desde 2013 o LHC não produz mais eventos, pois passa por uma fase de mudanças estruturais a fim de aumentar a luminosidade e energia do experimento. Estão previstos novos resultados em 2015 com energia do centro de massas $\sqrt{s}$ entre $13 \mathrm{TeV}$ e $14 \mathrm{TeV}$. Para ilustrar a 
nova fase de dados futuramente gerados pelo LHC, são apresentados resultados das seções de choque com a energia do centro de massas a $14 \mathrm{TeV}$.

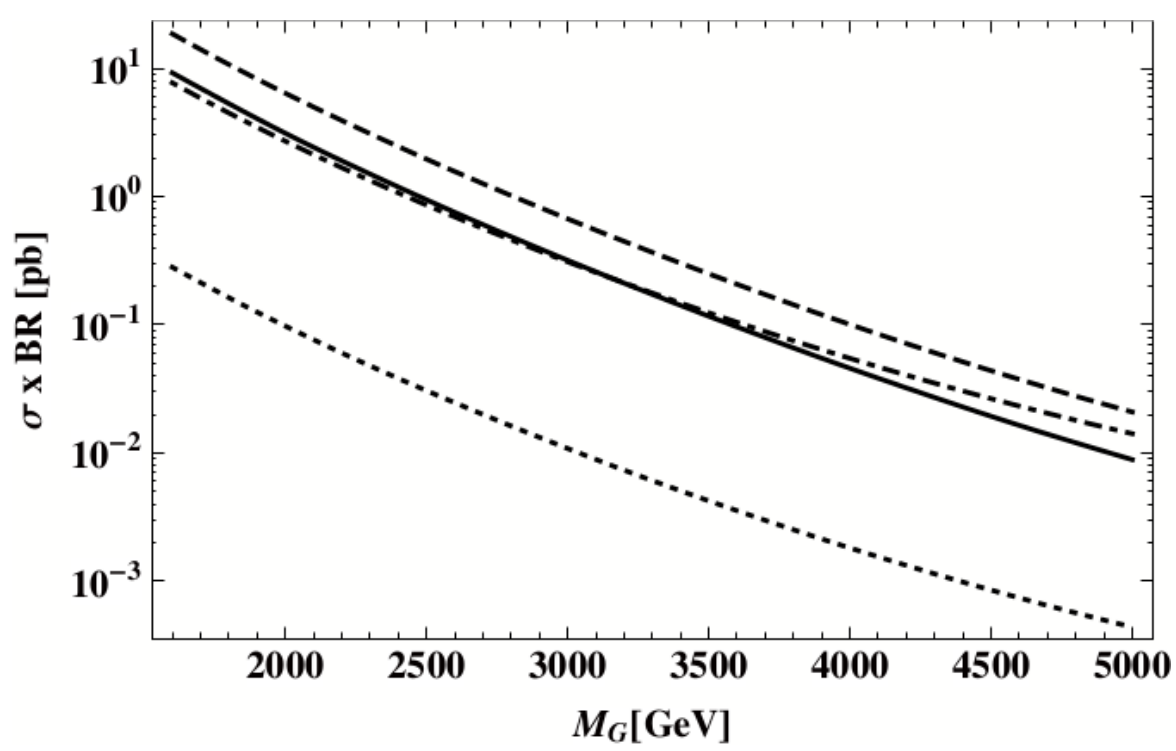

Figura 4.10: Seção de Choque multiplicada pela razão de ramificação em função da massa do primeiro estado excitado do glúon.As linhas correspondentes ao decaimento em jatos com número de sítios com $N=4$ é sólida e $N=15$ pontilhada. Enquanto as linhas tracejada corresponde a $N=4$ e $N=15$ pontilhada tracejada, ambas representam o estado final em $t \bar{t}$.Todas a $14 \mathrm{TeV}$. Figura publicada em [1].

Logo, foi escolhido $\sqrt{s}=14 \mathrm{TeV}$ para exemplificar os futuros dados obtidos pelo LHC, apresentados nas figuras 4.10 e 4.11 . Onde a primeira mostra os resultados para o primeiro estado excitado do octeto de cor intermediando as interações dos quarks do modelo padrão, enquanto a segunda figura representa interações intermediadas pelo singleto de cor $\left(\gamma^{\prime}+Z^{\prime}\right)$. Ambas as figuras mostram seções de choque multiplicada pela razão de ramificação para uma teoria a 5 e 16 sítios, decaindo tanto em $t \bar{t}$ quanto em jatos de quarks leves. Foram feitas essas escolhas com o propósito de ilustrar a fenomenologia das Teorias Quiver em poucos sítios descrita a partir dos próximos dados que serão obitdos a uma maior energia do centro de massas do processo. Ao analisar as figuras 4.10 e 4.11, observa-se que o número de eventos a massas maiores que $3 \mathrm{TeV}$ serão significativos na próxima fase do LHC, gerando vínculos mais restritos do que os vínculos indiretos de sabor e precisão eletrofraca. Por exemplo, a curva correspondente ao estado excitado do glúon a 5 sítios no quiver terá uma seção de choque, para sua massa de aproximadamente $5 \mathrm{TeV}$, a qual corresponderá a um número da ordem $10^{3}$ eventos, tomando a luminosidade integrada mínima de $300 \mathrm{fb}^{-1}$. 


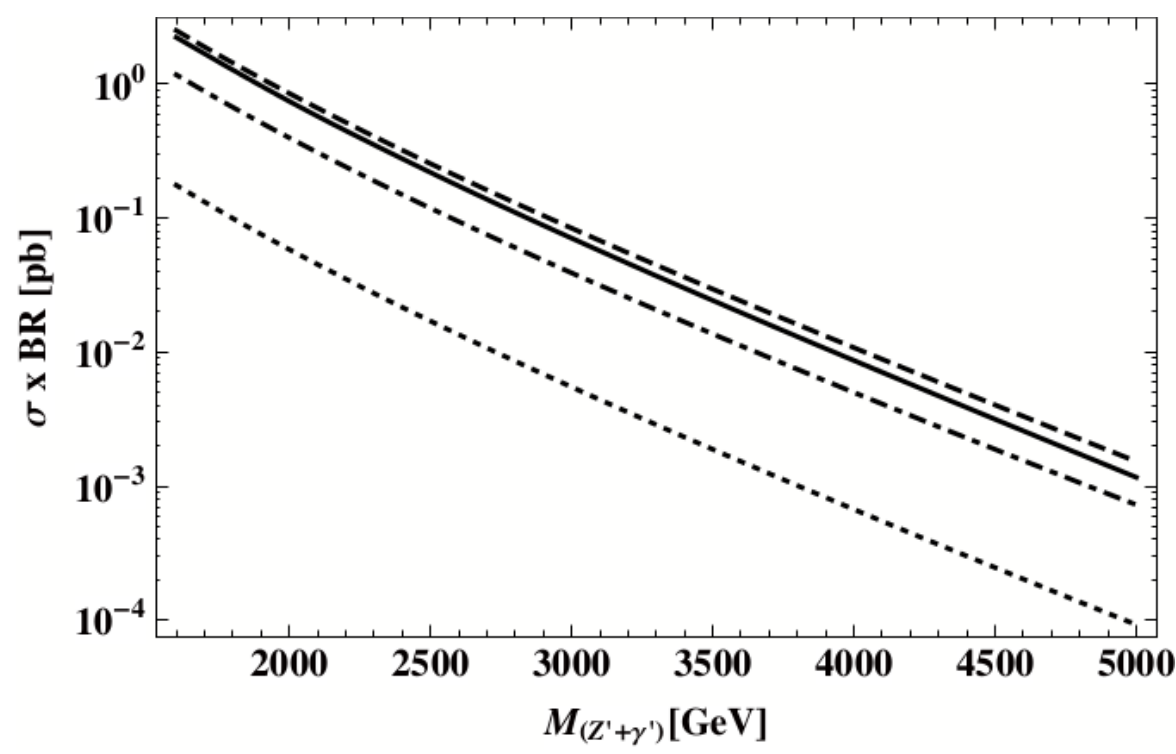

Figura 4.11: Seção de Choque multiplicada pela razão de ramificação em função da massa do primeiro estado excitado do glúon.As linhas correspondentes ao decaimento em jatos com número de sítios com $N=4$ é sólida e $N=15$ pontilhada.Enquanto as linhas tracejada corresponde a $N=4$ e $N=15$ pontilhada tracejada,ambas representam o estado final em $t \bar{t}$.Todas a $14 \mathrm{TeV}$.Figura publicada em [1].

\subsection{Conclusões}

Neste trabalho foi estudada a fenomenologia de Teorias Quiver quadridimensionais através de vínculos diretos obtidos do LHC, tanto para o primeiro modo excitado do octeto de cor quanto para o análogo eletrofraco. Esta teoria depende do número de sítios $\mathrm{N}$ nos diagramas quiver, que foi tratado como um parâmetro livre. No entanto, para $\mathrm{N}$ grandes, a teoria tende a um cenário de teorias com uma dimensão extra em $A d S_{5}, \log$, foi escolhido $N \leq 36$. [1], especificamente $N=5,10$ e 16 . Ao analisar os resultados apresentados nas Tabelas 4.14 .2 , pode-se chegar à conclusão de que os vínculos de massa para ambos os casos, do octeto de cor e $\left(Z^{\prime}+\gamma^{\prime}\right)$, são dominantes em $t \bar{t}$ e a teoria é competitiva em jatos para menores valores de $\mathrm{N}$.

Os vínculos de procura direta ainda não são melhores que os indiretos obtidos por medidas de precisão eletrofraca ou física de sabor. Porém, o LHC com $\sqrt{s}=14 \mathrm{TeV}$ e Luminosidade que irão de $300 \mathrm{fb}^{-1}$ a $3000 \mathrm{fb}^{-1}$ será capaz de obter vínculos muito além de $3 \mathrm{TeV}$ para as massas dos bósons de gauge excitados. Além disso, os valores dos vínculos para o singleto de cor, apresentados na Tabela 4.2 são baixos, e devem ser mais significantes para os canais de decaimentos em léptons. Estas teorias estão sendo desenvolvidas pelo grupo de pesquisa [19]. 


\section{Conclusões Finais}

O capítulo 1 consistiu em uma introdução à Física de Partículas. Foi apresentado o Modelo Padrão, discutindo aspectos teóricos necessários para a compreensão geral do texto. Além disso, questões além do MP foram mencionadas com o enfoque no problema de hierarquia, o qual é a maior motivação para a procura de nova física na escala TeV. Logo, as Teorias Quiver foram descritas como solução do problema de hierarquia, sendo o objetivo do trabalho estudar sua fenomenologia no LHC.

As Teorias Quiver são demonstradas no capítulo 2. Elas foram descritas como uma cadeia linear de sítios com grupos de simetria conectados por campos de ligação. A quebra espontânea através dos campos de ligação gera uma torre de estados excitados dos bósons de gauge. Ao incluir férmions na teoria, a hierarquia de massas é gerada naturalmente. Teorias Quiver podem ser derivadas de teorias com uma dimensão extra compacta através do processo e desconstrução. Entretanto, por ser puramente quadridimensionis, as Teorias Quiver com poucos sítios têm fenomenologia distinta do limite do contínuo.

No capítulo 3, são calculados parâmetros físicos necessários para demonstrar a fenomenologia de Teorias Quiver com poucos sítios. Também foi demonstrado que ao escrever o Higgs como um pseudo bóson de Nambu Goldstone é possível resolver o problema de hierarquia, mantendo a partícula localizada no IV. Em trabalhos recentes [25], foram calculados os vínculos de precisão eletrofraco para modelos em poucos sítios, chegando-se à conclusão que eles restringem a massa do primeiro estado excitado do bóson de gauge a $M_{G}>3 \mathrm{TeV}$.

Finalmente, no capítulo 4 foram feitos os cálculos da fenomenologia das Teorias Quiver em poucos sítios. Foram calculadas as seções de choque de produção vezes a razão de ramificação do decaimento do primeiro estado excitado dos bósons de gauge. Deste modo foi possível obter vínculos a partir de dados acumulados pelo LHC, publicados em [3] [4] [2] à energia do centro de massas $8 \mathrm{TeV}$. Os vínculos também foram discutidos na referência [1] .

Desse trabalho concluí-se que os vínculos de procura direta com $\sqrt{s}=8 \mathrm{TeV}$ são 
menos restringentes que os vínculos indiretos obtidos de experimentos de precisão eletrofraca e física de sabor, o que é aproximadamente $3 \mathrm{TeV}$. No entanto, o LHC com $14 \mathrm{TeV}$ e luminosidades entre $300 \mathrm{fb}^{-1}$ e $3000 \mathrm{fb}^{-1}$ terão uma sensitividade a massas ainda maiores. Por essa razão, a próxima fase do LHC terá vínculos de detecção direta melhores que os indiretos pela primeira vez.

\subsection{Perspectivas}

Foi apresentados os vínculos de massa para o octeto e singleto de cor das Teorias Quiver. É necessário aumentar a energia e luminosidade dos experimentos do LHC a fim de procurar de novas ressonâncias ou restringir com maior precisão esses vínculos. Para isso, foram feitos os cálculos da próxima fase dos experimentos que terão pouco menos de $14 \mathrm{TeV}$, como discutido na seção 4.5.2.

Por outro lado, resta ainda o estudo da fenomenologia em outros canais. É certo que o setor leptônico se acopla com os bósons de gauge sem cor. Deve-se calcular as seções de choque do decaimento dos modos de modos excitados para esse setor. Esta será comparada com dados acumulados pelo LHC a $8 \mathrm{TeV}$ que já foram publicados, por exemplo em [43]. Este trabalho já está em andamento no grupo de pesquisa [19]. Além disso, a fenomenologia dos quarks excitados tem sido estudada na referência [44]. 


\section{Referências Bibliográficas}

[1] Gustavo Burdman, Nayara Fonseca, and Gabriela Lichtenstein. Resonances from Quiver Theories at the LHC. Phys.Rev., D88:116006, 2013.

[2] CMS collaboration. Search for Heavy Resonances Decaying into bb and bg Final States in pp Collisions at sqrt(s) $=8 \mathrm{TeV} .2012$.

[3] Serguei Chatrchyan et al. Search for narrow resonances using the dijet mass spectrum in pp collisions at sqrt(s) $=8$ TeV. Phys.Rev., D87:114015, 2013.

[4] The ATLAS collaboration. A search for tt resonances in the lepton plus jets final state with ATLAS of pp collisions at $\operatorname{sqrt}(\mathrm{s})=8 \mathrm{TeV} .2013$.

[5] M.E. Peskin and D.V. Schroeder. An Introduction to Quantum Field Theory. Advanced book classics. Addison-Wesley Publishing Company, 1995.

[6] C. Quigg. Gauge Theories of the Strong, Weak, and Electromagnetic Interactions: Second Edition. Princeton University Press, 2013.

[7] J. Beringer et al. (Particle Data Group). Gauge and higgs bosons, PR D86, 010001 (2012) and 2013 partial update for the 2014 edition.

[8] F. Halzen and A.D. Martin. Quarks and leptons: an introductory course in modern particle physics. Wiley, 1984.

[9] F. Englert and R. Brout. Broken symmetry and the mass of gauge vector mesons. Phys. Rev. Lett., 13:321-323, Aug 1964.

[10] Peter W. Higgs. Broken symmetries and the masses of gauge bosons. Phys. Rev. Lett., 13:508-509, Oct 1964.

[11] G. S. Guralnik, C. R. Hagen, and T. W. B. Kibble. Global conservation laws and massless particles. Phys. Rev. Lett., 13:585-587, Nov 1964.

[12] S. Weinberg. The Quantum Theory of Fields: Volume 2, Modern Applications. Cambridge University Press, 1996.

[13] S.L. Glashow. Partial Symmetries of Weak Interactions. Nucl.Phys., 22:579-588, 1961. 
[14] Steven Weinberg. A model of leptons. Phys. Rev. Lett., 19:1264-1266, Nov 1967.

[15] Y. Sakai (Particle Data Group) A. Ceccucci, Z. Ligeti. 11 the ckm quark-mixing matrix, Revised March 2012.

[16] S.T. Petcov (Particle Data Group) K. Nakamura. 13. neutrino mass, mixing and oscilation, Updated May 2012.

[17] T.P. Cheng and L.F. Li. Gauge Theory of Elementary Particle Physics. Oxford science publications. Clarendon Press, 1984.

[18] Boris Kayser. Neutrino Mass, Mixing, and Flavor Change. 2008.

[19] Gabriela Lichtenstein Leonardo de Lima Camila Machado Gustavo Burdman, Nayara Fonseca and Ricardo Matheus. Fenomenologia das teorias quiver no setor leptonico, em preparação.

[20] J. Beringer et al. (Particle Data Group). Leptons, PR D86, 010001 (2012) and 2013 partial update for the 2014 edition.

[21] J. Beringer et al. (Particle Data Group). Quarks, PR D86, 010001 (2012) and 2013 partial update for the 2014 edition.

[22] Georges Aad et al. Observation of a new particle in the search for the Standard Model Higgs boson with the ATLAS detector at the LHC. Phys.Lett., B716:1-29, 2012.

[23] Serguei Chatrchyan et al. Observation of a new boson at a mass of $125 \mathrm{GeV}$ with the CMS experiment at the LHC. Phys.Lett., B716:30-61, 2012.

[24] M. Baak et al. The global electroweak fit at NNLO and prospects for the LHC and ILC. Eur.Phys.J., C74:3046, 2014.

[25] Gustavo Burdman, Nayara Fonseca, and Leonardo de Lima. Full-hierarchy Quiver Theories of Electroweak Symmetry Breaking and Fermion Masses. JHEP, 1301:094, 2013.

[26] Leonardo de Lima. Novos modelos na escala tev para a hierarquia de gauge e das massas dos férmions. Tese de doutorado, Instituto de Física da Universidade de São Paulo.

[27] Nayara Fonsceca de Sá. Desconstrução dimensional e violação de sabor. Dissertação de mestrado, Instituto de Física da Universidade de São Paulo.

[28] Jorge de Blas, Adam Falkowski, Manuel Perez-Victoria, and Stefan Pokorski. Tools for deconstructing gauge theories in AdS(5). JHEP, 0608:061, 2006.

[29] S. Pokorski e J. Wang C.T.Hill. Gauge invariant effective lagrangian for kaluzaklein modes. Physical Review D, 64(105005). 
[30] A. G. Cohen e H. Georgi N. Arkanni-Hammed. (de)constructing dimensions. Physical Review Letters, 86:4757-4761.

[31] Yang Bai, Gustavo Burdman, and Christopher T. Hill. Topological Interactions in Warped Extra Dimensions. JHEP, 1002:049, 2010.

[32] Lisa Randall, Yael Shadmi, and Neal Weiner. Deconstruction and gauge theories in AdS(5). JHEP, 0301:055, 2003.

[33] Tony Gherghetta. Les Houches lectures on warped models and holography. pages 263-311, 2006.

[34] Tony Gherghetta and Alex Pomarol. Bulk fields and supersymmetry in a slice of AdS. Nucl.Phys., B586:141-162, 2000.

[35] Adam Falkowski and Hyung Do Kim. Running of gauge couplings in AdS(5) via deconstruction. JHEP, 0208:052, 2002.

[36] M. Bona et al. Model-independent constraints on $\Delta F=2$ operators and the scale of new physics. JHEP, 0803:049, 2008.

[37] J.F. Donoghue, E. Golowich, and B.R. Holstein. Dynamics of the Standard Model. Cambridge Monographs on Particle Physics, Nuclear Physics and Cosmology. Cambridge University Press, 1994.

[38] J. Erler and P. Langacker (Particle Data Group). 10. electroweak model and constraints on new physics, Revised December 2011.

[39] K.G. Chetyrkin, Johann H. Kuhn, and M. Steinhauser. RunDec: A Mathematica package for running and decoupling of the strong coupling and quark masses. Comput.Phys.Commun., 133:43-65, 2000.

[40] V. Barger and R. Phillips. Collider Physics. Advanced book program. AddisonWesley Publishing Company, 1997.

[41] A.D. Martin, W.J. Stirling, R.S. Thorne, and G. Watt. Parton distributions for the LHC. Eur.Phys.J., C63:189-285, 2009.

[42] Georgios Choudalakis. On hypothesis testing, trials factor, hypertests and the bumphunter, arxiv: 1101.0390.

[43] Georges Aad et al. Search for $W Z$ resonances in the fully leptonic channel using $p p$ collisions at $\sqrt{s}=8 \mathrm{TeV}$ with the ATLAS detector. Phys.Lett., B737:223-243, 2014.

[44] Gustavo Burdman, Pedro Ormonde, and Victor Peralta. Fermion Resonances in Quiver Theories with a pNGB Higgs. 2014. 